\title{
WestVirginiaUniversity
}

THE RESEARCH REPOSITORY @ WVU

Graduate Theses, Dissertations, and Problem Reports

2017

\section{Integer Flows and Circuit Covers of Graphs and Signed Graphs}

Jian Cheng

Follow this and additional works at: https://researchrepository.wvu.edu/etd

\section{Recommended Citation}

Cheng, Jian, "Integer Flows and Circuit Covers of Graphs and Signed Graphs" (2017). Graduate Theses, Dissertations, and Problem Reports. 5354.

https://researchrepository.wvu.edu/etd/5354

This Dissertation is protected by copyright and/or related rights. It has been brought to you by the The Research Repository @ WVU with permission from the rights-holder(s). You are free to use this Dissertation in any way that is permitted by the copyright and related rights legislation that applies to your use. For other uses you must obtain permission from the rights-holder(s) directly, unless additional rights are indicated by a Creative Commons license in the record and/ or on the work itself. This Dissertation has been accepted for inclusion in WVU Graduate Theses, Dissertations, and Problem Reports collection by an authorized administrator of The Research Repository @ WVU.

For more information, please contact researchrepository@mail.wvu.edu. 


\title{
Integer Flows and Circuit Covers of Graphs and Signed Graphs
}

\author{
Jian Cheng \\ Dissertation submitted to the \\ Eberly College of Arts and Sciences \\ at West Virginia University \\ in partial fulfillment of the requirements \\ for the degree of
}

Doctor of Philosophy

in

Mathematics

Cun-Quan Zhang, Ph.D, Chair

Rong Luo, Ph.D, Co-chair

John L. Goldwasser, Ph.D

Hong-Jian Lai, Ph.D

James J. Nolan, Ph.D.

Jerzy Wojciechowski, Ph.D

Department of Mathematics

Morgantown, WV

2017

Keywords: Integer Flows; Circuit Covers; Signed Graphs; Vector Flows;

Even Factors

Copyright 2017 Jian Cheng 


\section{ABSTRACT}

\section{Integer Flows and Circuit Covers of Graphs and Signed Graphs}

\section{Jian Cheng}

The work in Chapter 2 is motivated by Tutte and Jaeger's pioneering work on converting modulo flows into integer-valued flows for ordinary graphs. For a signed graphs $(G, \sigma)$, we first prove that for each $k \in\{2,3\}$, if $(G, \sigma)$ is $(k-1)$-edge-connected and contains an even number of negative edges when $k=2$, then every modulo $k$-flow of $(G, \sigma)$ can be converted into an integer-valued $(k+1)$-flow with a larger or the same support. We also prove that if $(G, \sigma)$ is odd- $(2 p+1)$-edge-connected, then $(G, \sigma)$ admits a modulo circular $\left(2+\frac{1}{p}\right)$-flows if and only if it admits an integer-valued circular $\left(2+\frac{1}{p}\right)$-flows, which improves all previous result by Xu and Zhang (DM2005), Schubert and Steffen (EJC2015), and Zhu (JCTB2015).

Shortest circuit cover conjecture is one of the major open problems in graph theory. It states that every bridgeless graph $G$ contains a set of circuits $\mathcal{F}$ such that each edge is contained in at least one member of $\mathcal{F}$ and the length of $\mathcal{F}$ is at most $\frac{7}{5}|E(G)|$. This concept was recently generalized to signed graphs by Máčajová et al. (JGT2015). In Chapter 3, we improve their upper bound from $11|E(G)|$ to $\frac{14}{3}|E(G)|$, and if $G$ is 2-edgeconnected and has even negativeness, then it can be further reduced to $\frac{11}{3}|E(G)|$.

Tutte's 3-flow conjecture has been studied by many graph theorists in the last several decades. As a new approach to this conjecture, DeVos and Thomassen considered the vectors as flow values and found that there is a close relation between vector $S^{1}$-flows and integer 3-NZFs. Motivated by their observation, in Chapter 4, we prove that if a graph $G$ admits a vector $S^{1}$-flow with rank at most two, then $G$ admits an integer 3 -NZF.

The concept of even factors is highly related to the famous Four Color Theorem. We conclude this dissertation in Chapter 5 with an improvement of a recent result by Chen and Fan (JCTB2016) on the upperbound of even factors. We show that if a graph $G$ contains an even factor, then it contains an even factor $H$ with $|E(H)| \geq \frac{4}{7}(|E(G)|+1)+\frac{1}{7}\left|V_{2}(G)\right|$, where $V_{2}(G)$ is the set of vertices of degree two. 


\section{Acknowledgements}

I would like to express my deep appreciation and gratitude to my supervisors, Dr. CunQuan Zhang and Dr. Rong Luo, for their continued encouragement and support over these last few years. It is a great pleasure to work under their supervision. Without their guidance and persistent help, this dissertation would not have been possible.

I would also like to thank my other committee members: Dr. John Goldwasser, Dr. Hong-Jian Lai, Dr. James J. Nolan and Dr. Jerzy Wojciechowski, for their help during my studies in West Virginia University.

Finally, I would like to thank the Department of Mathematics and Eberly College of Arts and Sciences at West Virginia University for providing me with an excellent study environment and support during my study as a graduate student. 


\section{DEDICATION}

To

my father Zhijun Cheng and my mother Bingying Li

and

my wife Keke and my son Sean 


\section{Contents}

1 Introduction 1

1.1 Notation and terminology $\ldots \ldots \ldots \ldots \ldots \ldots$

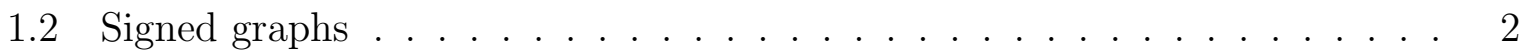

1.3 Integer-valued flows on signed graphs $\ldots \ldots \ldots \ldots \ldots$

1.3.1 Two analogs of Tutte's flow theorem . . . . . . . . . . . . . 4

1.3.2 A generalization of Jaeger's circular flow theorem . . . . . . . 6

1.4 Shortest circuit covers of signed graphs . . . . . . . . . . . . . . . 8

1.5 Vector flows on graphs $\ldots \ldots \ldots \ldots$

1.6 Even factors of graphs . . . . . . . . . . . . . . . . . . . . 12

2 Flows on Signed Graphs $\quad 15$

2.1 Proof of Theorem $1.3 .6 \ldots \ldots \ldots \ldots \ldots$

2.2 Proof of Theorem $1.3 .7 \ldots \ldots \ldots \ldots \ldots$

2.3 Proof of Corollary 1.3.9 . . . . . . . . . . . . . . . . . . . . . . . . 19 
2.4 Proof of Theorem $1.3 .15 \ldots \ldots \ldots \ldots$

2.4 .1 A new vertex splitting lemma . . . . . . . . . . . 20

2.4.2 Application of Tutte's factor theorem . . . . . . . . . . . . . . 21

2.4.3 Completion of the Proof of Theorem 1.3.15 . . . . . . . . 25

3 Shortest Circuit Covers of Signed Graphs 26

3.1 Notation and Terminology . . . . . . . . . . . . . . . . 26

3.2 Lemmas and outline of the proofs $\ldots \ldots \ldots \ldots \ldots$

3.3 Signed circuit covers of generalized barbells . . . . . . . . . . . . . 28

3.4 Proof of Lemma $3.2 .3 \ldots \ldots \ldots$

3.5 Proof of Theorem $3.2 .2 \ldots \ldots \ldots \ldots$

4 Vector Flows and Integer Flows 41

4.1 Notation and Terminology . . . . . . . . . . . . . . . . . . 41

4.2 Vector $S^{1}$-flows of the dual of unit distance graph $\ldots \ldots \ldots \ldots$. . . . 42

4.3 Proof of Theorem $1.5 .7 \ldots \ldots \ldots \ldots 4 \ldots$

4.3.1 Vector $S^{1}$-flows with rank one . . . . . . . . . . 44

4.3 .2 Some useful lemmas . . . . . . . . . . . . . . . . . 46

4.3.3 Vector $S^{1}$-flows with rank two . . . . . . . . . . . 52

4.4 Proof of Theorem $1.5 .9 \ldots \ldots \ldots \ldots \ldots$

$5 \quad$ Even Factors of Graphs $\quad 59$ 
$5.1\{\mathrm{~B}, \mathrm{Y}, \mathrm{R}\}$-edge-coloring of $G \ldots \ldots \ldots \ldots$

$5.2 Q$-subgraphs in $R \cup R^{*} \cup B \ldots \ldots \ldots \ldots$

5.3 Charge and Discharge .................... . . 65

5.4 The Final Step . . . . . . . . . . . . . . . . . . 68 


\section{Chapter 1}

\section{Introduction}

\subsection{Notation and terminology}

Graphs in this dissertation are undirected, finite, and may have multiple edges or loops. Readers are referred to $[4,8,50]$ for undefined terminology and notations. Let $G$ be a graph with vertex set $V(G)$ and edge set $E(G)$. For each vertex $v \in V(G)$, the set of vertices adjacent to $v$ (known as the neighborhood of $v$ ) and the set of edges incident with $v$ are respectively denoted by $N(v)$ and $E(v)$, and the degree of $v$ is $\operatorname{deg}(v)=|E(v)|$. A cycle is a connected subgraph of $G$ in which each vertex has even degree, and a circuit refers to a minimal cycle of $G$.

For each edge subset $F \subseteq E(G)$, we use $G-F$ to denote the subgraph of $G$ obtained by deleting all edges in $F$, and use $G / F$ to denote the graph obtained from $G$ by identifying the two ends of each edge in $F$ and then deleting the resulting loops.

For each vertex subset $X \subseteq V(G)$, we use $G[X]$ to denote the subgraph of $G$ with vertex set $X$ and edge set $\{u v \in E(G): u, v \in X\}$, and use $G-X$ to denote the subgraph of $G$ by deleting the vertices in $X$ together with the edges incident with at least one vertex in $X$. For any two disjoint subsets $A, B$ of $V(G)$, we use $E(A, B)$ to denote the set of edges with one end in $A$ and the other end in $B$, and $e(A, B)$ to denote their number. 
A vertex $v$ is a cut-vertex if $G-\{v\}$ has more components than $G$. An edge set $F$ is a $\lambda$-edge cut if $|F|=\lambda$ and $G-F$ has more components than $G$. A graph $G$ is odd$\lambda$-edge-connected if it contains no odd- $(\lambda-2)$-edge cut. The odd-edge-connectivity of $G$ is the smallest integer $\lambda$ for which $G$ is odd- $\lambda$-edge-connected. If $F=\{e\}$, we simply call $e$ a bridge of $G$. A graph $G$ is bridgeless if $G$ contains no bridges.

For any two subgraphs $H, K$ of $G$, we respectively use $H \cup K$ and $H \cap K$ to denote the subgraphs of $G$ induced by $E(H) \cup E(K)$ and $E(H) \cap E(K)$, and the symmetric difference of $H$ and $K$, denoted $H \triangle K$, is the subgraph of $G$ induced by $(H \cup K)-(H \cap K)$.

\section{$1.2 \quad$ Signed graphs}

A signed graph is a graph $G$ associated with a signature $\sigma: E(G) \rightarrow\{-1,1\}$. An edge $e \in E(G)$ is positive if $\sigma(e)=1$ and negative otherwise. The set of negative edges of $(G, \sigma)$ is denoted by $E_{N}(G, \sigma)$. For signed graphs $(G, \sigma)$, every edge $e \in E(G)$ consists of two half-edges, each of which is incident with one end of $e$. For each vertex $v \in V(G)$, we use $H(v)$ to denote the set of all half-edges incident with $v$. Let $H(G)=\bigcup_{v \in V(G)} H(v)$. For each half-edge $h \in H(G)$, we use $e_{h}$ to denote the parent edge containing $h$. An orientation of $(G, \sigma)$ is a mapping $\tau: H(G) \rightarrow\{-1,1\}$ such that $\tau\left(h_{1}\right) \tau\left(h_{2}\right)=-\sigma(e)$ for each edge $e$ of $G$, where $h_{1}$ and $h_{2}$ are two half-edges contained in $e$.

A circuit of $G$ is balanced if it contains an even number of negative edges and unbalanced otherwise. To generalize the concept of shortest circuit covers to signed graphs, we introduced the concept of signed circuits, which is defined as follows (see Figure 1.1):

- either a balanced circuit,

- or the union of two edge-disjoint unbalanced circuits meeting at exactly one single vertex (known as a short barbell),

- or the union of two disjoint unbalanced circuits together with a path that meets the circuits only at its ends (known as a long barbell). 
A signed graph $(G, \sigma)$ is $s$-bridgeless if each edge of $G$ is contained in a signed circuit. The length of a signed circuit $C$ is the number of edges in $C$.

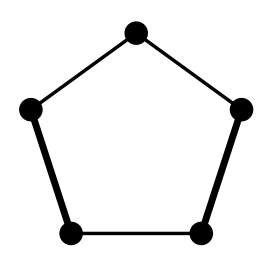

A balanced circuit

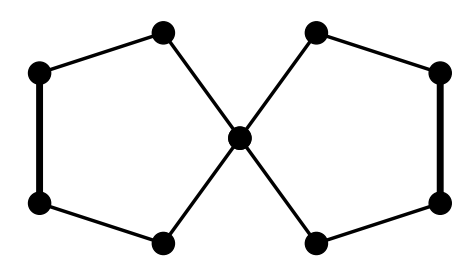

A short barbell

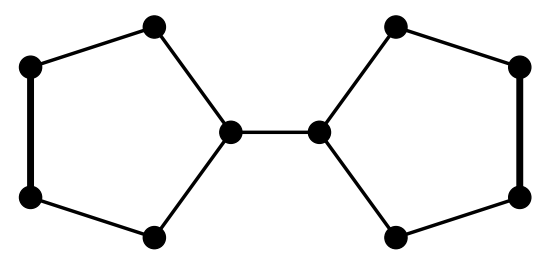

A long barbell

Figure 1.1: Three types of signed circuits

For a signed graph $(G, \sigma)$, switching at a vertex $v$ means changing the sign of each edge incident to $v$ such that in the resulting signed graph $(G, \sigma) \sigma^{\prime}(e)=-\sigma(e)$ for each edge $e \in E(v)$ and $\sigma^{\prime}(e)=\sigma(e)$ for all other edges. See Figure 1.2 for all illustration. Two signatures are said to be equivalent if one can be obtained from the other by making a sequence of switchings. Let $\mathcal{X}_{(G, \sigma)}$ be the set of signatures (of $G$ ) equivalent to $\sigma$. The negativeness of $(G, \sigma)$ is defined as the smallest integer $q$ for which $G$ has a signature $\sigma^{\prime} \in \mathcal{X}_{(G, \sigma)}$ with exactly $q$ negative edges, and is denoted by $\epsilon_{N}(G, \sigma)$.

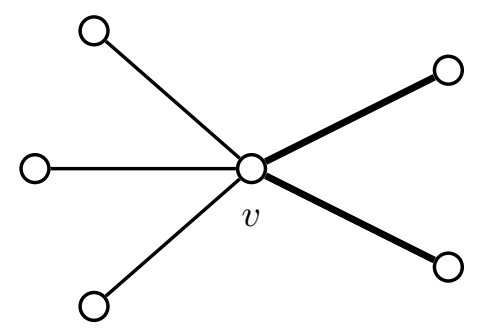

$(G, \sigma)$

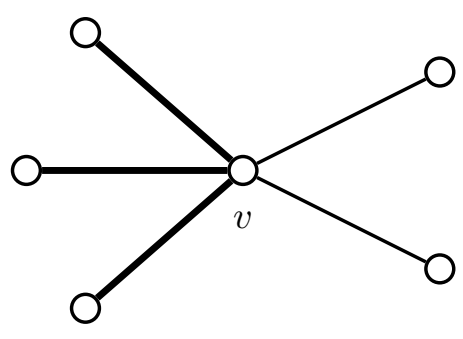

$\left(G, \sigma^{\prime}\right)$

Figure 1.2: A switch at vertex $v$ (bold edges are negative edges)

\subsection{Integer-valued flows on signed graphs}

In the flow theory, an integer-valued flow and a modulo flow are different by their definitions. For graphs (equivalently, signed graph with all edges positive), Tutte [47] 
showed that a graph admits a nowhere-zero integer-valued $k$-flow if and only if it admits a nowhere-zero modulo $k$-flow. We also notice that most landmark results were initially proved for modulo flows (such as, the 8-flow theorem by Jaeger [20], the 6-flow theorem by Seymour [42], the weak 3-flow theorem by Thomassen [44], etc.) and stated as an integer-valued flow results due to the theorem by Tutte.

However, Tutte's result [47] cannot be applied for signed graphs (see Figure 1.3). That is, there is a big gap between modulo flows and integer-valued flows for signed graphs. The first known result was proved by Bouchet [5] in his study of chain-group.

Theorem 1.3.1 ([5], Proposition 3.5). If a signed graph $(G, \sigma)$ admits a modulo $k$-flow $f_{1}$, then it admits an integer-valued $2 k$-flow $f_{2}$ with $\operatorname{supp}\left(f_{1}\right) \subseteq \operatorname{supp}\left(f_{2}\right)$.

In this dissertation Theorem 1.3.1 is improved for some important cases: modulo 2-flows, modulo 3 -flows, and modulo circular $\left(2+\frac{1}{p}\right)$-flows.

\subsubsection{Two analogs of Tutte's flow theorem}

Definition 1.3.2. Let $(G, \sigma)$ be a signed graph associated with an orientation $\tau$. Let $k$ be a positive integer and $f$ be an integer-valued mapping defined on $E(G)$ such that $0 \leq$ $|f(e)| \leq(k-1)$. The boundary of $f$ at a vertex $v$ is defined as $\partial f(v)=\sum_{h \in H(v)} f\left(e_{h}\right) \tau(h)$. The mapping $f$ is a modulo $k$-flow of $(G, \sigma)$ if $\partial f(v) \equiv 0(\bmod k)$ for each vertex $v$ of $G$, and is an integer-valued $k$-flow of $(G, \sigma)$ if $\partial f(v)=0$ for each vertex $v$ of $G$.

Let $(G, \sigma)$ be a signed graph and $f$ be a flow of $(G, \sigma)$. The support of $f$, denoted by $\operatorname{supp}(f)$, is the set of edges $e$ with $f(e) \neq 0$. A flow $f$ is nowhere-zero if $\operatorname{supp}(f)=E(G)$. For convenience, we respectively shorten the notations of nowhere-zero flows into integervalued $k$-NZFs and modulo $k$-NZFs. A signed graph $(G, \sigma)$ is said to be flow-admissible if it admits an integer-valued $k$-NZF.

It is well-known that the admission of nowhere-zero flows is independent of its orientation. For a signed graph $(G, \sigma)$, for convenience, we can always assume its orientation is $\tau$ without mentioning it. 


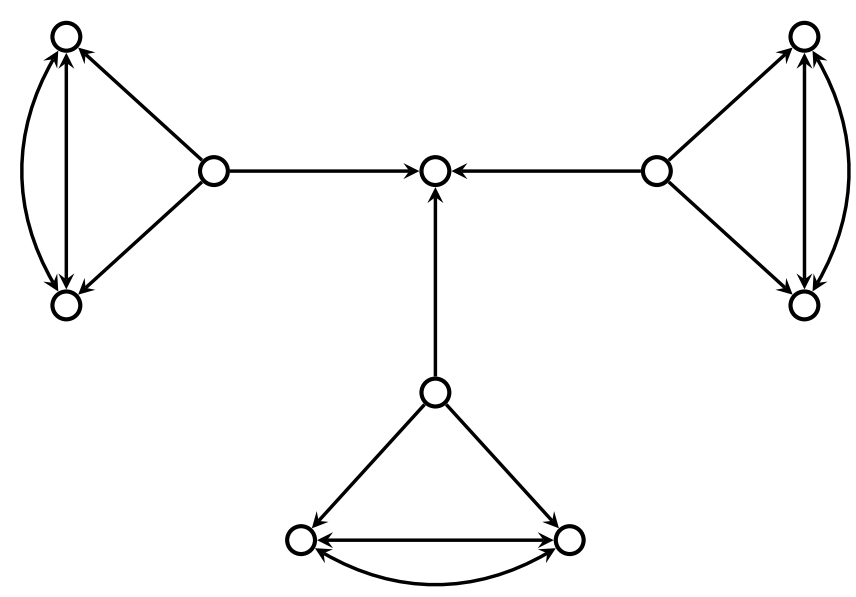

Figure 1.3: $(G, \sigma)$ admits a modulo 3-NZF, but no integer-valued 3-NZF.

In 1949, Tutte made the following observation.

Theorem 1.3.3 (Tutte [47]). A graph $G$ admits a modulo $k-N Z F$ if and only if it admits an integer-valued $k-N Z F$.

To verify Bouchet's 6-flow conjecture [5] for 6-edge-connected signed graphs, Xu and Zhang [51] proved the following two results, which generalize Tutte's theorem to signed graph with $k=2,3$.

Theorem 1.3.4 (Xu and Zhang [51]). If a signed graph $(G, \sigma)$ admits a modulo 2-flow $f_{1}$ such that each component of $\operatorname{supp}\left(f_{1}\right)$ contains an even number of negative edges, then it also admits an integer-valued 2-flow $f_{2}$ with $\operatorname{supp}\left(f_{1}\right)=\operatorname{supp}\left(f_{2}\right)$.

Theorem 1.3.5 (Xu and Zhang [51]). If a signed graph $(G, \sigma)$ admits a modulo 3-flow $f_{1}$ such that $\operatorname{supp}\left(f_{1}\right)$ is bridgeless, then it also admits an integer-valued 3 -flow $f_{2}$ with $\operatorname{supp}\left(f_{1}\right)=\operatorname{supp}\left(f_{2}\right)$.

In this dissertation, under the weaker conditions, we prove the following two results which are analogs of Theorem 1.3.1 and respectively improve Theorem 1.3.4 and 1.3.5.

Theorem 1.3.6. If a signed graph $(G, \sigma)$ admits a modulo 2-flow $f_{1}$ such that $\operatorname{supp}\left(f_{1}\right)$ contains an even number of negative edges, then it also admits an integer-valued 3-flow $f_{2}$ with $\operatorname{supp}\left(f_{1}\right)=\left\{e \in E(G): f_{2}(e)= \pm 1\right\}$. 
Theorem 1.3.7. If a signed graph $(G, \sigma)$ is bridgeless and admits a modulo 3-flow $f_{1}$, then it also admits an integer-valued 4-flow $f_{2}$ with $\operatorname{supp}\left(f_{1}\right) \subseteq\left\{e \in E(G): f_{2}(e)= \pm 1, \pm 2\right\}$.

The concept of flows on signed graphs arises naturally as a dual of local tensions on non-orientable surfaces. In 1983, Bouchet [5] proposed the following well-known 6 -flow conjecture, which motivated most of the studies in this area.

Conjecture 1.3.8 (Bouchet [5], 6-flow conjecture). Every flow-admissible signed graph admits an integer-valued $6-N Z F$.

This conjecture remains widely open and Bouchet [5] himself proved it is true with 6 replaced by 216. The best published result is 30-flow by Zýka [57]. Khelladi [27] showed that for 4-edge-connected graphs, the upper bound can be reduced to 18 and was improved to 4 by Raspaud and Zhu in [40]. Xu and Zhang [51] confirmed it for 6-edge-connected signed graphs. Recently, DeVos [7] improved Zýka's result to 12-flow, which is the best approach until today. Readers are also referred to a recent survey by Kaiser et al. [26] for more discussions.

As an application of Theorem 1.3.6 and 1.3.7, we further improve DeVos' result to integer-valued 11-NZFs for bridgeless signed graphs.

Corollary 1.3.9. Every bridgeless flow-admissible signed graph admits an integer-valued $11-N Z F$.

\subsubsection{A generalization of Jaeger's circular flow theorem}

Definition 1.3.10. Let $(G, \sigma)$ be a signed graph associated with an orientation $\tau$.

- Let $k$ and $d$ be two positive integers. An integer-valued (respectively modulo) circular $\frac{k}{d}$-flow of $(G, \sigma)$ is an integer-valued (respectively modulo) flow $f$ such that $d \leq|f(e)| \leq k-d$ for each edge $e \in E(G)$.

- Let $p$ be a positive integer. The orientation $\tau$ is a modulo $(2 p+1)$-orientation if $\sum_{e \in H(v)} \tau(e) \equiv 0(\bmod 2 p+1)$ for each vertex $v \in V(G)$. 
When $k=3$, Tutte's theorem [47] implies that a graph $G$ admits a modulo circular 3 -flow if and only if it admits an integer-valued circular 3-flow. This result was generalized to integer-valued circular $\left(2+\frac{1}{p}\right)$-flows by Jaeger [21] as follows.

Theorem 1.3.11 (Jaeger [21]). Let $G$ be a graph and $p$ be a positive integer. Then the following statements are equivalent:

(A) $G$ admits a modulo $(2 p+1)$-orientation.

(B) G admits a modulo circular $\left(2+\frac{1}{p}\right)$-flow.

(C) G admits an integer-valued circular $\left(2+\frac{1}{p}\right)$-flow.

For signed graphs, using an identical proof in [21], we can prove that (A) and (B) are still equivalent. However, similar to modulo flows, the equivalent relation between (B) and $(\mathbf{C})$ does not hold for signed graphs (see Firgure 1.3). For more details, readers are referred to [25], [34], [40], [41], [51], [56], etc.

The following are some early results on the equivalent relation between (B) and (C) for signed graphs.

Theorem 1.3.12 (Xu and Zhang [51]). If a signed graph $(G, \sigma)$ is cubic and contains a perfect matching, then it admits a modulo circular 3-flow if and only if it admits an integer-valued circular 3-flow.

Theorem 1.3.13 (Schubert and Steffen [41]). If a signed graph $(G, \sigma)$ is $(2 p+1)$-regular and contains a p-factor, then it admits a modulo circular $\left(2+\frac{1}{p}\right)$-flow if and only if it admits an integer-valued circular $\left(2+\frac{1}{p}\right)$-flow.

Theorem 1.3.14 (Zhu [56]). If a signed graph $(G, \sigma)$ is $(12 p-1)$-edge-connected with negativeness even or at least $2 p+1$, then it admits a modulo circular $\left(2+\frac{1}{p}\right)$-flow if and only if it admits an integer-valued circular $\left(2+\frac{1}{p}\right)$-flow.

In this dissertation, we generalize all results above as follows.

Theorem 1.3.15. If a signed graph $(G, \sigma)$ is odd-(2p+1)-edge-connected, then it admits a modulo circular $\left(2+\frac{1}{p}\right)$-flow if and only if it admits an integer-valued circular $\left(2+\frac{1}{p}\right)$-flow. 


\subsection{Shortest circuit covers of signed graphs}

A circuit cover of a bridgeless graph $G$ is a family $\mathcal{C}$ of circuits such that each edge of $G$ belongs to at least one member of $\mathcal{C}$. The length of $\mathcal{C}$ is the total length of circuits in $\mathcal{C}$ and the minimum length of circuit covers of $G$ is denoted by $\operatorname{SCC}(G)$.

For ordinary graphs (signed graphs with all edges positive), the subject of shortest circuit cover is not only a discrete optimization problem [17], but also closely related to some mainstream areas in graph theory, such as, Tutte's integer flow theory $[1,3,10,18$, 23, 37, 52], circuit double cover conjecture [24, 28], Fulkerson conjecture [11], snarks and graph minors $[2,19]$.

In 1985, Alon and Tarsi [1] proposed the following conjecture that motivates the most of the studies in this area.

Conjecture 1.4.1 (Alon and Tarsi [1]). Every bridgeless graph $G$ has $\operatorname{SCC}(G) \leq \frac{7|E(G)|}{5}$.

The following are some early results related to Conjecture 1.4.1

Theorem 1.4.2 (Bermond, Jackson, and Jaeger [3]). Every graph $G$ admitting an integervalued $4-N Z F$ has $\operatorname{SCC}(G) \leq \frac{4|E(G)|}{3}$.

Theorem 1.4.3 (Alon and Tarsi [1], and Bermond, Jackson, and Jaeger [3]). Every bridgeless graph $G$ has $\operatorname{SCC}(G) \leq \frac{5|E(G)|}{3}$.

Theorem 1.4.4 (Jamshy and Tarsi [24]). Conjecture 1.4.1 implies the Circuit Double Cover Conjecture.

Theorem 1.4.5 (Fan [12]). Every 2-edge-connected graph $G$ has $\operatorname{SCC}(G) \leq|E(G)|+$ $|V(G)|-1$.

The relations between $\operatorname{SCC}(G)$ and Fulkerson conjecture, Tutte's 3-flow and 5-flow conjectures were studied by Fan, Jamshy, Raspaud, and Tarsi in [11, 10, 23].

It is natural to generalize the concept of shortest circuit covers from bridgeless graphs to $s$-bridgeless signed graphs. That is, let signed circuits in signed graphs play the role of 
circuits in graphs. It is obvious (see [40]) that the switching operation preserves signed circuits and thus the existence and the length of a signed circuit cover of a signed graph are two invariants under the switching operation. For the topic of signed circuit covers, it is convenient to say that $G$ is a signed graph without mentioning the signature and $\operatorname{SCC}(G)$ denotes the minimum length of signed circuit covers of $G$. Recently, the following upper bounds for $\operatorname{SCC}(G)$ were first estimated in [36].

Theorem 1.4.6 (E. Máčajová, A. Raspaud, E. Rollová, M. Škoviera [36]). Let $G=(V, E)$ be an s-bridgeless signed graph. Then $\operatorname{SCC}(G) \leq 11|E|$. Furthermore, if $G$ is 2-edgeconnected, then $\operatorname{SCC}(G) \leq 9|E|$.

In this dissertation, we further improve Theorem 1.4.6 as follows.

Theorem 1.4.7. Let $G=(V, E)$ be an s-bridgeless signed graph with negativeness $\epsilon_{N}>0$.

(1) In general, we have

$$
\operatorname{SCC}(G) \leq|E|+3|V|+\min \left\{\frac{2}{3}|E|+\frac{4}{3} \epsilon_{N}-7,|V|+2 \epsilon_{N}-8\right\} .
$$

(2) If $G$ is 2-edge-connected and $\epsilon_{N}$ is even, then

$$
\operatorname{SCC}(G) \leq|E|+2|V|+\min \left\{\frac{2}{3}|E|+\frac{1}{3} \epsilon_{N}-4,|V|+\epsilon_{N}-5\right\} .
$$

\section{Remark.}

(a) Theorem 1.4.7 is an analog of Theorem 1.4.5 by Fan [12] that solves a long standing open problem proposed by Itai and Rodeh [17].

(b) For a connected $s$-bridgeless signed graph $G, G-E_{N}(G)$ is a connected ordinary graph (by Lemma 3.4.3) and therefore $|E| \geq \epsilon_{N}+|V|-1$. Hence, if $G$ is an $s$-bridgeless signed graph with $\epsilon_{N}>0$, by Theorem 1.4.7-(1), then

$$
\operatorname{SCC}(G) \leq \frac{14}{3}|E|-\frac{5}{3} \epsilon_{N}-4<\frac{14}{3}|E| .
$$

This is an analog of Theorem 1.4.3 by Alon and Tarsi [1] and by Bermond, Jackson, and Jaeger [3]. 


\subsection{Vector flows on graphs}

The concept of integer-valued flows on ordinary graphs (signed graphs with all edges positive) was introduced by Tutte as a dual of map coloring problem. One of the major open problems in this area is Tutte's 3-flow conjecture: every 4-edge-connected graph admits an integer-valued 3-NZF. Extended from a recent breakthrough in [44], this conjecture has been verified for all odd 7-edge-connected graphs [32].

Theorem 1.5.1 (Lovász, Thomassen, Wu, and Zhang [32]). Every odd-7-edge-connected graph admits an integer-valued 3-NZF.

Definition 1.5.2. Let $T$ be a subset of vectors in the Euclidian space $\mathbb{R}^{n}$ and $G$ be a graph with an orientation $D$. An ordered pair $(D, \boldsymbol{\Phi})$ is a vector $T$-flow of $G$ if $\boldsymbol{\Phi}: E(G) \rightarrow T$ such that, for each vertex $v$ of $G$,

$$
\sum_{e \in E_{D}^{+}(v)} \boldsymbol{\Phi}(e)=\sum_{e \in E_{D}^{-}(v)} \boldsymbol{\Phi}(e),
$$

where $E_{D}^{+}(v)$ and $E_{D}^{-}(v)$ are the sets of edges oriented away from and towards to $v$. See Figure 1.4 for an illustration.

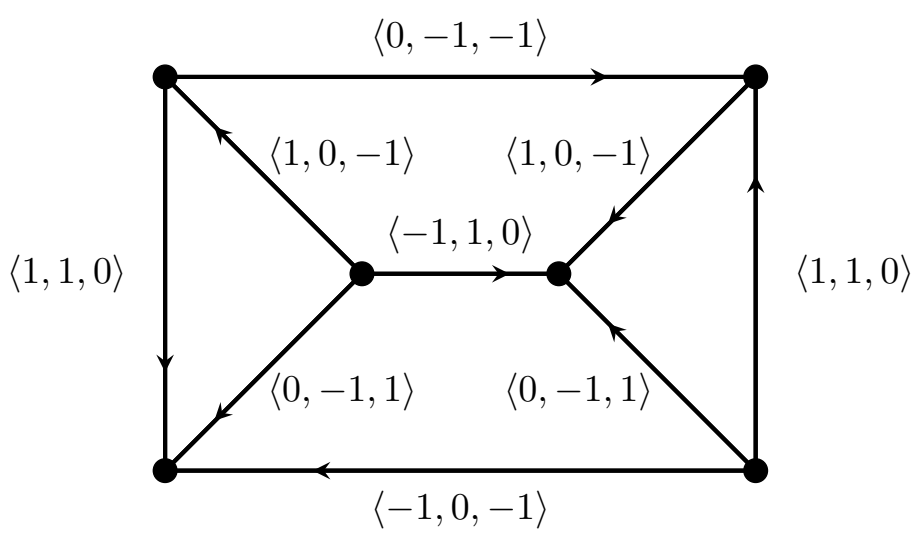

Figure 1.4: A vector $S^{2}$-flow of a graph $G$

Let $S^{1}$ denote the set of all vectors $\boldsymbol{\alpha}$ in $\mathbb{R}^{2}$ with $\|\boldsymbol{\alpha}\|=1$. The following conjecture motivates most of our research in this area. 
Conjecture 1.5.3 (Jain [22], vector $S^{1}$-flow conjecture). Every 4-edge-connected graph admits a vector $S^{1}$-flow.

DeVos [22] and Thomassen [45] proved that a graph admits a vector $S^{1}$-flow if it admits an integer-valued 3-NZF. Thomassen [45] pointed out that a graph admitting a vector $S^{1}$-flow may not necessarily admit an integer-valued 3-NZF and presented a family of examples showing that the converse is not true.

In this dissertation, we follow [45] and study the relation between the two graphic properties: integer-valued 3-NZFs and vector $S^{1}$-flows.

I. A graph $G$ admits an integer-valued 3-NZF.

II. A graph $G$ admits a vector $S^{1}$-flow.

III. A graph $G$ admits a vector $C_{3}$-flow where $C_{3}$ consists of three complex roots of the unit.

It was asked by DeVos [22] whether I and II are equivalent. The following theorem responds to this question and unveils some relations between these two graphic properties.

Theorem 1.5.4 (Thomassen [45]).

(1) I and III are equivalent.

(2) I implies II, but not vise versa.

(3) I and II are equivalent for cubic graphs.

Recall that Thomassen [45] discovered a family of graphs that admit vector $S^{1}$-flows but no integer-valued 3-NZFs. See Section 4.2 for the detailed discussion. Due to the existence of such counterexamples, it is natural to ask the following problem.

Problem 1.5.5. Characterize vector $S^{1}$-flows for which $\mathbf{I I}$ implies $\mathbf{I}$. That is, if $G$ admits a vector $S^{1}$-flow (not necessary a vector $C_{3}$-flow) with certain properties, then $G$ also admits an integer-valued $3-N Z F$. 
The vector $C_{3}$-flow (described in III) is one of such examples for which II implies I. Problem 1.5.6. Characterize graphs for which II implies I. That is, if $G$ is a graph with certain properties and admits a vector $S^{1}$-flow, then $G$ also admits an integer-valued $3-N Z F$.

The following is one of our main theorems, which is a partial solution to Problem 1.5.5.

Theorem 1.5.7. If a graph $G$ admits a vector $S^{1}$-flow with rank at most two, then $G$ admits an integer-valued $3-N Z F$.

Note that all the vector $C_{3}$-flows (in III) are vector $S^{1}$-flows with rank one. Theorem 1.5.7, in some sense, is an extension of Theorem 1.5.4-(1) and is the study of the converse of Theorem 1.5.4-(2). Theorem 1.5.7 is sharp in the sense that there are graphs that admit vector $S^{1}$-flows with rank three, but no integer-valued 3-NZFs (discovered by Thomassen [45], see Section 4.2). In fact, we prove a stronger result as follows.

Theorem 1.5.8. If a graph $G$ admits a vector $S^{1}$-flow with rank at most two, then either $G$ is eulerian and thus admits an integer-valued 2-NZF, or $G$ admits an integer-valued circular $\left(2+\frac{1}{p}\right)$-flow for some positive integer $p$.

The following result is motivated by Problem 1.5.6 and extends Theorem 1.5.4-(3).

Theorem 1.5.9. Let $G$ be a graph and $V_{3}$ be the set of vertices of degree 3 in $G$. If $G\left[V_{3}\right]$ is connected and $G-V_{3}$ is acyclic, then $\mathbf{I}$ and $\mathbf{I I}$ are equivalent (That is, $G$ admits an integer-valued 3-NZF if and only if $G$ admits a vector $S^{1}$-flow).

Remark. In one of Thomassen's examples (see Figure 4.1-(a)), it is easy to see that $G\left[V_{3}\right]$ has two components. Thus the condition that $G\left[V_{3}\right]$ is connected in Theorem 1.5.9 may not be dropped.

\subsection{Even factors of graphs}

An even factor of $G$ is a spanning subgraph of $G$ in which each vertex has a positive even degree. If an even factor is 2-regular, then we call it a 2-factor of $G$. In the following 
context, a $l$-circuit (resp. $(\geq l)$-circuit) is a circuit whose length is $l$ (resp. at least $l$ ).

Petersen's theorem [39] says that every bridgeless cubic graph $G$ has a 2-factor $F$. In [16], Fleischner improved this result and showed that if $G$ is a bridgeless graph with minimum degree at least three, then $G$ has an even factor.

By applying the Matching Polytope Theorem [9] and Splitting Lemma [14], the size of a maximum even factor was first estimated by Lai and Chen [29].

Theorem 1.6.1 (Lai and Chen [29]). If $G$ is a bridgeless graph with minimum degree at least three, then $G$ has an even factor $F$ such that $|E(F)| \geq \frac{2}{3}|E(G)|$.

Relaxing the requirements of bridgeless and minimum degree for graphs, Favaron and Kouider [13] proved the following result.

Theorem 1.6.2 (Favaron and Kouider [13]). If a graph $G$ has an even factor, then it has an even factor $F$ such that $|E(F)| \geq \frac{9}{16}(|E(G)|+1)$.

Chen and Fan [6] recently improved this ratio to $\frac{4}{7}$, which is the best possible. The extremal graphs can be obtained from trees by blowing up each vertex with pairwise disjoint $K_{4}$ (complete graph of order 4 ).

Theorem 1.6.3 (Chen and Fan [6]). If a graph $G$ has an even factor, then it has an even factor $F$ such that $|E(F)| \geq \frac{4}{7}(|E(G)|+1)$.

Our interest in this problem was motivated by the relation between $V_{2}(G)$ (the set of vertices of degree two) and even factors. Let $F$ be any maximum even factor of a graph $G$ and subdivide one edge of $F$. Note that $F$ generates an even factor $F^{\prime}$ of the resulting graph $G^{\prime}$ and the numbers of edges of $F$ and $G$ both increase by 1 . The inequality in Theorem 1.6.3 still holds, but the gap between the left side and right side is larger with the increase in $\left|V_{2}(G)\right|$. Based on these observations, we conjecture that when $\left|V_{2}(G)\right|$ increases, the size of a maximum even factor gets larger.

Conjecture 1.6.4. There exists a constant $c>0$ such that every graph $G$ having an even factor $F$ will have an even factor $H$ such that $|E(H)| \geq \frac{4}{7}(|E(G)|+1)+c\left|V_{2}(G)\right|$. 
In [6], Chen and Fan introduced an optimized edge-coloring technique. In this dissertation, we adopt this technique and further introduce the concept of $Q$-subgraphs. Using the discharging method, we confirm aforementioned conjecture and strengthen Theorem 1.6.3 as follows.

Theorem 1.6.5. If a graph $G$ has an even factor $F$, then it has an even factor $H$ such that $|E(H)| \geq \frac{4}{7}(|E(G)|+1)+\frac{1}{7}\left|V_{2}(G)\right|$.

Remark. The family of extremal graphs in Theorem 1.6.3 shows that the first part of the lower bound in Equation (5.8) (that is, $\frac{4}{7}(|E(G)|+1)$ ) cannot be improved any more. We do not know whether the coefficient $1 / 7$ of $\left|V_{2}(G)\right|$ is best possible or not, but the example of Figure 1.5 shows that it cannot be larger than $2 / 7$. Note that $G^{*}$ has a maximum even factor $H^{*}$ consisting of 4 -circuits and satisfying

$$
\left|E\left(H^{*}\right)\right|=\frac{4}{7}\left(\left|E\left(G^{*}\right)\right|+1\right)+\frac{2}{7}\left|V_{2}\left(G^{*}\right)\right| .
$$

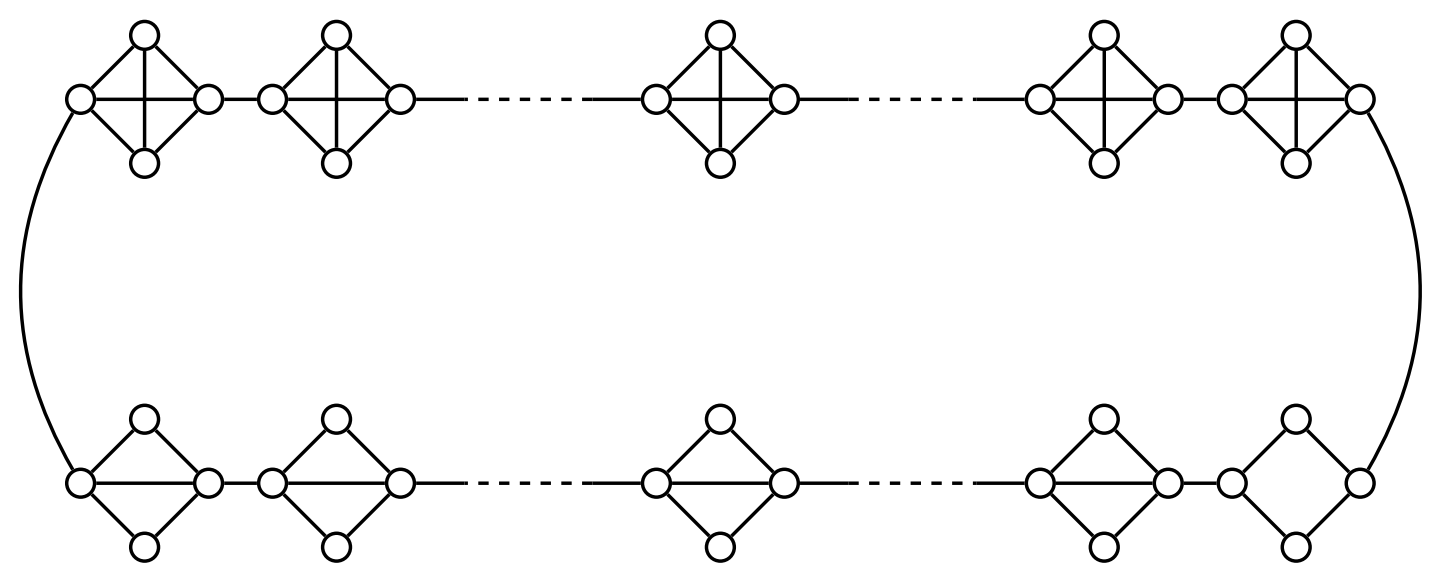

Figure 1.5: $G^{*}$ 


\section{Chapter 2}

\section{Flows on Signed Graphs}

\subsection{Proof of Theorem 1.3.6}

Let $(G, \sigma)$ together with a flow $f_{1}$ be a counterexample to Theorem 1.3.6 such that $|E(G)|$ is minimized. Thus, $G$ must be connected. In each of the following context, we are to yield a contradiction by showing that $(G, \sigma)$ actually admits an integer-valued 3-flow $f_{2}$ satisfying Theorem 1.3.6. For convenience, let $B=\operatorname{supp}\left(f_{1}\right)$.

Claim 1. $B \neq E(G)$ and each edge of $E(G)-B$ is a bridge.

Proof. If $B=E(G)$, then $G$ is an eulerian graph containing an even number of negative edges. By Theorem 1.3.4, $G$ admits an integer-valued 2-NZF, say $f_{2}$. If $e^{*} \in E(G)-B$ is not a bridge of $G$, let $G^{\prime}=G-\left\{e^{*}\right\}$, then $f_{1}$ is a modulo 2-flow of $G^{\prime}$ with $\left|E\left(G^{\prime}\right)\right|<|E(G)|$. Thus, $\left(G^{\prime}, \sigma\right)$ admits an integer-valued 3-flow $f_{2}$ such that $B=\left\{e \in E\left(G^{\prime}\right): f_{2}(e)= \pm 1\right\}$. In both cases, $f_{2}$ is an integer-valued 3-flow of $G$ satisfying Theorem 1.3.6.

Claim 2. For an edge $e \in E(G)-B$, denote by $Q_{1}$ and $Q_{2}$ the components of $G-\{e\}$. Then neither $B \cap Q_{1}$ nor $B \cap Q_{2}$ contains an even number of negative edges.

Proof. Since $B$ contains an even number of negative edges, $B \cap Q_{1}$ and $B \cap Q_{2}$ contain the same parity number of negative edges. Suppose to the contrary that each contains an even 
number of negative edges. For $i \in\{1,2\}$, it follows from $\left|E\left(Q_{i}\right)\right|<|E(G)|$ that $\left(Q_{i}, \sigma\right)$ admits an integer-valued 3-flow $g_{i}$ such that $B \cap Q_{i}=\left\{e \in E\left(Q_{i}\right): g_{i}(e)= \pm 1\right\}$. One can verify that $f_{2}=g_{1}+g_{2}$ is an integer-valued 3-flow of $G$ satisfying Theorem 1.3.6.

Now we first choose an edge $e^{*} \in E(G)-B$ and denote its endpoints by $x_{1}$ and $x_{2}$, respectively. Let $Q_{1}$ and $Q_{2}$ be the components of $G-\left\{e^{*}\right\}$ such that $x_{1} \in V\left(Q_{1}\right)$ and $x_{2} \in V\left(Q_{2}\right)$. For $i \in\{1,2\}$, let $\left(H_{i}, \sigma_{i}\right)$ be the resulting signed graph obtained from $Q_{i}$ by adding a negative loop $e_{i}$ at $x_{i}$. Denote $B_{i}=\left(B \cap Q_{i}\right) \cup\left\{e_{i}\right\}$. It follows from Claim 2 that $B_{i}$ contains an even number of negative edges. Assigning $f_{1}\left(e_{i}\right)=1$ and thus $f_{1}$ is a modulo 2-flow of $\left(H_{i}, \sigma_{i}\right)$ with support $B_{i}$. Note that $\left|E\left(H_{i}\right)\right|<|E(G)|$. Thus, $\left(H_{i}, \sigma_{i}\right)$ admits an integer-valued 3-flow $g_{i}$ such that $B_{i}=\left\{e \in E\left(H_{i}\right): g_{i}(e)= \pm 1\right\}$. Note that $\left|\partial g_{i}\left(x_{i}\right)\right|=2$ for each component $Q_{i}$. We can without lose of generality assume $\partial g_{2}\left(x_{2}\right)=-\sigma\left(e^{*}\right) \partial g_{1}\left(x_{1}\right)$ since otherwise we can replace $g_{1}$ by $-g_{1}$. Now we define $f_{2}$ by assigning $f_{2}(e)=g_{i}(e)$ if $e \in E\left(Q_{i}\right)$ and $f_{2}\left(e^{*}\right)=2$ (or -2 ) (such that the boundaries of $f_{2}$ at $x_{1}$ and $x_{2}$ are both zero). Finally, $f_{2}$ is an integer-valued 3-flow of $G$ satisfying Theorem 1.3.6.

\subsection{Proof of Theorem 1.3.7}

First let us recall the vertex-spliting operation and Splitting Lemma.

Definition 2.2.1 (Vertex Splitting). Let $G$ be a graph and $v$ be a vertex of $G$. Suppose $F \subset E(v)$, then we denote $G_{(v ; F)}$ to be the graph obtained from $G$ by splitting the edges of $F$ away from $v$. That is, adding a new vertex $v^{*}$ and changing the common endpoint of edges in F from $v$ to $v^{*}$. See Figure 2.1 for an illustration.

Lemma 2.2.2 (Splitting Lemma [14], or see [15]). Let $G$ be a bridgeless graph. If $v$ is a vertex with $\operatorname{deg}(v) \geq 4$ and $e_{1}, e_{2}, e_{3} \in E(v)$ are chosen in a way that $e_{1}$ and $e_{3}$ are in different blocks when $v$ is a cut-vertex, then either $G_{\left(v ;\left\{e_{1}, e_{2}\right\}\right)}$ or $G_{\left(v ;\left\{e_{1}, e_{3}\right\}\right)}$ is bridgeless. Furthermore, $G_{\left(v ;\left\{e_{1}, e_{3}\right\}\right)}$ has this property if $v$ is a cut-vertex. 


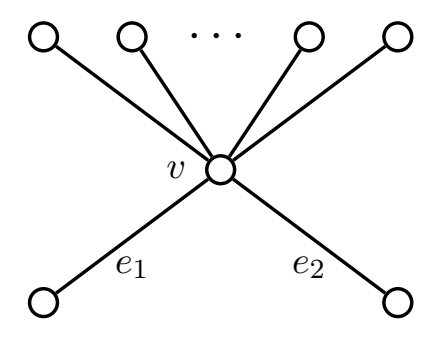

$G$

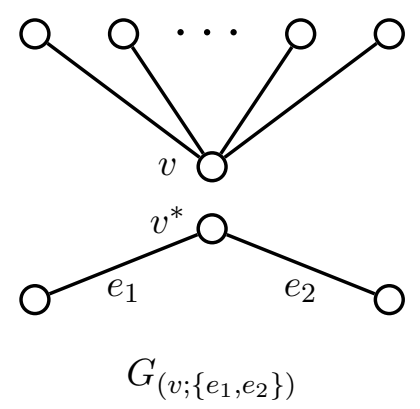

Figure 2.1: Splitting $\left\{e_{1}, e_{2}\right\}$ away from $v$

Proof of Theorem 1.3.7. Let $(G, \sigma)$ together with a flow $f_{1}$ be a counterexample to Theorem 1.3.7 such that

(1) $\left|\operatorname{supp}^{c}\left(f_{1}\right)\right|$ is minimized, where $\operatorname{supp}^{c}\left(f_{1}\right)=E(G)-\operatorname{supp}\left(f_{1}\right)$;

(2) subject to $(1), \sum_{v \in V(G)}|\operatorname{deg}(v)-3|$ is minimized.

Thus, $G$ must be connected. Similar to the proof of Theorem 1.3.6, we are to yield a contradiction by finding an integer-valued 4-flow satisfying Theorem 1.3.7.

Claim 3. $\operatorname{supp}\left(f_{1}\right) \neq \emptyset$ and $\operatorname{supp}^{\mathrm{c}}\left(f_{1}\right) \neq \emptyset$.

Proof. If $\operatorname{supp}\left(f_{1}\right)=\emptyset$, then simply let $f_{2}(e)=0$ for each edge $e$ of $G$. If $\operatorname{supp}^{\mathrm{c}}\left(f_{1}\right)=\emptyset$, then $\operatorname{supp}\left(f_{1}\right)=E(G)$ and thus $f_{1}$ is a modulo 3 -NZF of $(G, \sigma)$. Since $G$ is bridgeless, Theorem 1.3.5 implies that $(G, \sigma)$ admits an integer-valued $3-\mathrm{NZF} f_{2}$. In both cases, $f_{2}$ is an integer-valued 4-flow satisfying Theorem 1.3.7.

Claim 4. The maximum degree of graph $G$ is at most 3.

Proof. Suppose that $G$ has a vertex $v$ with $\operatorname{deg}(v) \geq 4$. Since $G$ is bridgeless, it follows from Lemma 2.2.2 that we can split a pair of edges $\left\{e_{1}, e_{2}\right\}$ from $v$ such that the resulting signed graph, say $\left(G_{1}, \sigma_{1}\right)$, is still bridgeless. Denote by $v^{*}$ the common endpoint of $e_{1}$ and $e_{2}$. In $G_{1}$, we have $\partial f_{1}\left(v^{*}\right) \equiv-\partial f_{1}(v)(\bmod 3)$. 
Choose $w \in\left\{v, v^{*}\right\}$. If $\partial f_{1}(w) \equiv 0(\bmod 3)$ and $\operatorname{deg}_{G_{1}}(w)=2\left(\right.$ say $E_{G_{1}}(w)=$ $\left.\left\{e_{w^{\prime}}, e_{w^{\prime \prime}}\right\}\right)$, then we further suppress the vertex $w$ and denote the resulting edge by $e_{w}$ (see Figure 2.2-(1)). Now we assign $e_{w}$ with value $f_{1}\left(e_{w^{\prime}}\right)$, signature $\sigma_{1}\left(e_{w^{\prime}}\right) \sigma_{1}\left(e_{w^{\prime \prime}}\right)$, and an orientation (based on its signature and value) such that both endpoints of $e_{w}$ have zero boundary. If $\partial f_{1}(w) \not \equiv 0(\bmod 3)$, then we add a positive edge $v v^{*}$, which is oriented from $v$ to $v^{*}$ and assigned with value $\partial f_{1}\left(v^{*}\right)$ (see Figure 2.2-(2)). In both cases, denote the resulting signed graph and mapping by $\left(G_{2}, \sigma_{2}\right)$ and $g_{1}$, respectively.

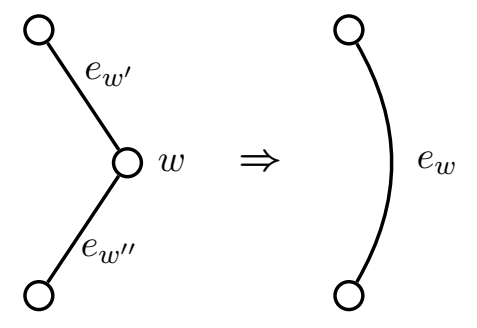

(1) $\partial f_{1}(w) \equiv 0(\bmod 3), \operatorname{deg}_{G_{1}}(w)=2$
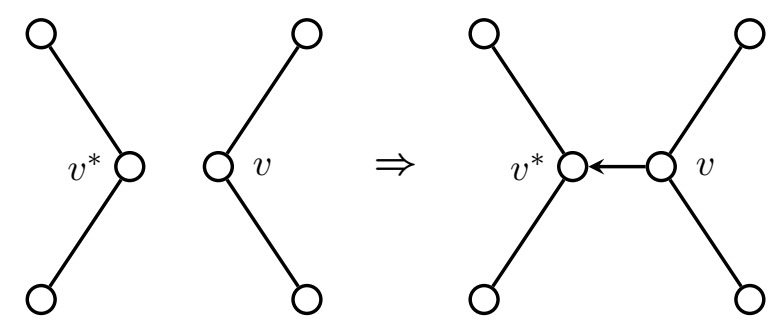

(2) $\partial f_{1}(w) \not \equiv 0(\bmod 3)$

Figure 2.2: Construction of signed graph $\left(G_{2}, \sigma_{2}\right)$

It is easy to see that $g_{1}$ is a modulo 3 -flow of $\left(G_{2}, \sigma_{2}\right)$ and $\left|\operatorname{supp}^{c}\left(g_{1}\right)\right| \leq\left|\operatorname{supp}^{c}\left(f_{1}\right)\right|$. It follows from $\sum_{v \in V\left(G_{2}\right)}\left|\operatorname{deg}_{G_{2}}(v)-3\right|<\sum_{v \in V(G)}|\operatorname{deg}(v)-3|$ that $\left(G_{2}, \sigma_{2}\right)$ has an integervalued 4-flow $g_{2}$ such that $\operatorname{supp}\left(g_{1}\right) \subseteq\left\{e \in E\left(G_{2}\right): g_{2}(e)= \pm 1, \pm 2\right\}$. It is trivial to derive an integer-valued 4 -flow $f_{2}$ of $(G, \sigma)$ from $g_{2}$, which satisfies Theorem 1.3.7.

Combining the fact that $G$ is connected and Claim 3, we know that $G$ has a vertex $x$ such that $E(x) \cap \operatorname{supp}\left(f_{1}\right) \neq \emptyset$ and $E(x) \cap \operatorname{supp}^{c}\left(f_{1}\right) \neq \emptyset$. Choose an edge $e^{*} \in$ $E(x) \cap \operatorname{supp}^{c}\left(f_{1}\right)$ and denote the other endpoint of $e$ by $y$. Without lose of generality, we can assume that $e^{*}$ is positive since otherwise we can make a switch at $x$, and that $e^{*}$ is oriented from $x$ to $y$. Furthermore, we contract $e^{*}$ and denote the resulting signed graph by $\left(G^{\prime}, \sigma^{\prime}\right)$. Thus, the restriction of $f_{1}$ to $E\left(G^{\prime}\right)$, denoted $f_{1}^{\prime}$, is a modulo 3-flow of $\left(G^{\prime}, \sigma^{\prime}\right)$. Note that $\operatorname{supp}\left(f_{1}^{\prime}\right)=\operatorname{supp}\left(f_{1}\right)$ implies $\left|\operatorname{supp}^{\mathrm{c}}\left(f_{1}^{\prime}\right)\right|<\left|\operatorname{supp}^{\mathrm{c}}\left(f_{1}\right)\right|$. Hence, $\left(G^{\prime}, \sigma^{\prime}\right)$ admits an integer-valued 4-flow $f_{2}^{\prime}$ such that $\operatorname{supp}\left(f_{1}^{\prime}\right) \subseteq\left\{e \in E\left(G^{\prime}\right): f_{2}^{\prime}(e)= \pm 1, \pm 2\right\}$.

Now we consider the mapping $f_{2}^{\prime}$ on $E(G)$. Each vertex (possibly except $x$ and $y$ ) has zero boundary and $\partial f_{2}^{\prime}(x)=-\partial f_{2}^{\prime}(y)$. If $\partial f_{2}^{\prime}(x) \not \equiv 0(\bmod 3)$, then we extend $f_{2}^{\prime}$ to 
a new function $h_{1}$ by assigning $h_{1}\left(e^{*}\right)=-\partial f_{2}^{\prime}(x)$. Note that $h_{1}$ is a modulo 3-flow of $G$ with $\operatorname{supp}\left(h_{1}\right) \supset \operatorname{supp}\left(f_{1}\right)$. This implies $\left|\operatorname{supp}^{c}\left(h_{1}\right)\right|<\left|\operatorname{supp}^{\mathrm{c}}\left(f_{1}\right)\right|$, which contradicts the assumption $(1)$. Thus, we can assume $\partial f_{2}^{\prime}(x) \equiv 0(\bmod 3)$. In summary, $x$ a vertex of $G$ satisfying $d(x) \leq 3, E(x) \cap \operatorname{supp}^{c}\left(f_{1}\right) \neq \emptyset$, and $1 \leq\left|f_{2}^{\prime}(e)\right| \leq 2$ for each edge $e$ in $E(x) \cap \operatorname{supp}\left(f_{1}\right)$. Hence, $0 \leq\left|\partial f_{2}^{\prime}(x)\right| \leq 4$ and furthermore $\left|\partial f_{2}^{\prime}(x)\right| \in\{0,3\}$. Finally, we can extend $f_{2}^{\prime}$ to a new mapping $f_{2}$ by assigning $f_{2}\left(e^{*}\right)=-\partial f_{2}^{\prime}(x)$. Clearly, $f_{2}$ is an integer-valued 4-flow satisfying Theorem 1.3.7.

\subsection{Proof of Corollary 1.3.9}

Lemma 2.3.1 ([20] or [42]). Let $G$ be an ordinary graph and $k_{1}, k_{2}$ be two integers. If $G$ admits an integer-valued $k_{1}$-flow $f_{1}$ and an integer-valued $k_{2}$-flow $f_{2}$ such that $\operatorname{supp}\left(f_{1}\right) \cup$ $\operatorname{supp}\left(f_{2}\right)=E(G)$, then both $k_{2} f_{1}+f_{2}$ and $f_{1}+k_{1} f_{2}$ are integer-valued $k_{1} k_{2}$-NZFs of $G$.

Remark. Lemma 2.3.1 can be naturally generalized to signed graphs.

Let $(G, \sigma)$ be a signed graph and $\phi=f_{1} \times g_{1}: E(G) \rightarrow \mathbb{Z}_{2} \times \mathbb{Z}_{3}$ be a flow of $(G, \sigma)$, where $f_{1}$ and $g_{1}$ respectively represent a modulo 2 -flow and modulo 3 -flow of $(G, \sigma)$. The flow $\phi$ is balanced if $\operatorname{supp}\left(f_{1}\right)$ contains an even number of negative edges. The following technical lemma was proved in the proof of $12-\mathrm{NZF}$ by DeVos [7].

Lemma 2.3.2 ([7]). Every flow-admissible signed graph admits a balanced $\mathbb{Z}_{2} \times \mathbb{Z}_{3}$-NZF.

Proof of Corollary 1.3.9. It follows from Lemma 2.3.2 that $(G, \sigma)$ admits a balanced $\mathbb{Z}_{2} \times \mathbb{Z}_{3}$-NZF $\phi=f_{1} \times g_{1}$. Now we apply Theorem 1.3.6 to $f_{1}$ and Theorem 1.3.7 to $g_{1}$. Then $(G, \sigma)$ admits an integer-valued 3-flow $f_{2}$ and an integer-valued 4-flow $g_{2}$ such that

$$
\begin{aligned}
& \operatorname{supp}\left(f_{1}\right)=\left\{e \in E(G):\left|f_{2}(e)\right|=1\right\} \\
& \operatorname{supp}\left(g_{1}\right) \subseteq\left\{e \in E(G):\left|g_{2}(e)\right|=1,2\right\} .
\end{aligned}
$$

By Lemma 2.3.1, $h=4 f_{2}+g_{2}$ is an integer-valued 12-NZF of $(G, \sigma)$. Here, we claim that $|h(e)| \neq 11$ for each edge $e$ in $G$. Suppose $|h(e)|=11$ for some edge $e \in E(G)$. Since 
$\left|f_{2}(e)\right| \leq 2,\left|g_{2}(e)\right| \leq 3$, and $h=4 f_{2}+g_{2}$, we have $\left|f_{2}(e)\right|=2$ and $\left|g_{2}(e)\right|=3$. But $\operatorname{supp}\left(f_{1}\right) \cup \operatorname{supp}\left(g_{1}\right)=E(G)$, it follows from Equations (2.1) and (2.2) that,

$$
\begin{aligned}
& \left\{e \in E(G):\left|g_{2}(e)\right|=3\right\} \subseteq \operatorname{supp}\left(f_{1}\right)-\operatorname{supp}\left(g_{1}\right) \\
& \left\{e \in E(G):\left|f_{2}(e)\right|=2\right\} \subseteq \operatorname{supp}\left(g_{1}\right)-\operatorname{supp}\left(f_{1}\right) .
\end{aligned}
$$

Clearly, such an edge $e$ does not exist and this completes the proof of Corollary 1.3.9.

\subsection{Proof of Theorem 1.3.15}

\subsubsection{A new vertex splitting lemma}

The vertex splitting method is one of the most useful techniques in graph theory (especially, in the studies of integer flow and cycle cover problems). In Section 2.2, we have discussed Splitting Lemma introduced by Fleischner (see Lemma 2.2.2). Here are more early results about vertex splitting.

Theorem 2.4.1 (Nash-Williams [38]). Let $\lambda$ be an even integer and $G$ be $a \lambda$-edgeconnected graph. Let $v$ be a vertex of $G$ and $a$ be an integer such that $\lambda \leq a$ and $\lambda \leq$ $\operatorname{deg}(v)-a$. Then there is an edge subset $F \subset E(v)$ such that $|F|=a$ and $G_{(v ; F)}$ remains $\lambda$-edge-connected.

Theorem 2.4.2 (Mader [35]). Let $G$ be a graph and $v \in V(G)$ such that $v$ is not a cutvertex of $G$. If $\operatorname{deg}(v) \geq 4$ and $v$ is adjacent to at least two distinct vertices, then there are two edges $e_{1}, e_{2} \in E(v)$ such that, for each pair of vertices $\{x, y\}$ in $V(G)-\{v\}$, the local edge-connectivity between $x$ and $y$ in the graph $G_{\left(v ;\left\{e_{1}, e_{2}\right\}\right)}$ remains the same as in $G$.

Theorem 2.4.3 (Zhang [54]). Let $G$ be a graph with odd-edge-connectivity at least $\lambda_{o}$ and $v \in V(G)$ such that $\operatorname{deg}(v) \neq \lambda_{o}$ and $E(v)=\left\{e_{0}, e_{1}, \ldots, e_{\operatorname{deg}(v)-1}\right\}$. Then there is a pair of edges $\left\{e_{i}, e_{i+1}\right\}$ in $E(v)$ with indices taken modulo $\operatorname{deg}(v)$ such that the graph $G_{\left(v ;\left\{e_{i}, e_{i+1}\right\}\right)}$ remains odd- $\lambda_{o}$-edge-connected.

Definition 2.4.4. Let $G$ be a graph and $v$ be a vertex of $G$. Suppose that $S(v)$ is a subset of $\left\{\left(e_{i}, e_{j}\right): e_{i}, e_{j} \in E(v)\right.$ and $\left.e_{i} \neq e_{j}\right\}$. The subset $S(v)$ is sequentially connected if, for 
each pair of edges $\left\{e^{\prime}, e^{\prime \prime}\right\}$ in $E(v)$, there is a sequence $\left(e_{0}, e_{1}\right),\left(e_{1}, e_{2}\right), \ldots,\left(e_{t-1}, e_{t}\right) \in S(v)$ $(\bmod \operatorname{deg}(v))$ such that $e^{\prime}=e_{0}$ and $e^{\prime \prime}=e_{t}$.

In Theorem 2.4.3, the subset $S(v)$ is taken to be $\left\{\left(e_{i}, e_{i+1}\right): i \in \mathbb{Z}_{\operatorname{deg}(v)}\right\}$. In this dissertation, using an identical proof of Theorem 2.4.3 in [54], we prove the following result which is expected to have many applications in graph theory. An alternative proof of Theorem 2.4.3 can be also found in [43].

Theorem 2.4.5. Let $G$ be a graph with odd-edge-connectivity at least $\lambda_{o}$ and $v \in V(G)$ such that $\operatorname{deg}(v) \neq \lambda_{o}$. Let $S(v)$ be a subset of $\left\{\left(e_{i}, e_{j}\right): e_{i}, e_{j} \in E(v)\right.$ and $\left.e_{i} \neq e_{j}\right\}$. If the subset $S(v)$ is sequentially connected, then there is a pair $\left(e^{\prime}, e^{\prime \prime}\right) \in S(v)$ such that the graph $G_{\left(v ;\left\{e^{\prime}, e^{\prime \prime}\right\}\right)}$ remains odd- $\lambda_{o}$-edge-connected.

Corollary 2.4.6 is an analog of Theorem 2.4.1 with respect to odd-edge-connectivity.

Corollary 2.4.6. Let $G$ be a graph with odd-edge-connectivity at least $\lambda_{o}$ and $v \in V(G)$ with $\operatorname{deg}(v)>\lambda_{o}$. Let $S(v)=\left\{\left(e_{i}, e_{j}\right): e_{i}, e_{j} \in E(v)\right.$ and $\left.e_{i} \neq e_{j}\right\}$. Let a be an even integer such that $a \leq \operatorname{deg}(v)-\lambda_{o}$. Then there is an edge subset $F \subset E(v)$ of size $a$ and consisting of disjoint elements of $S(v)$ such that $G_{(v ; F)}$ remains odd- $\lambda_{o}$-edge-connected.

Proof. Let $a=2 b$. Now we apply Theorem 2.4.5 to $v$ repeatedly $b$ times at $v$. Denote by $\left\{v_{1}^{*}, \ldots, v_{b}^{*}\right\}$ the set of resulted new vertices of degree two. We further identify these $b$ new vertices as a new vertex $v^{*}$. Note that the resulting graph, denoted by $G^{*}$, remains odd- $\lambda_{o}$-edge-connected. Thus, $E_{G^{*}}\left(v^{*}\right)$ is a desired edge subset $F$ of $E(v)$.

\subsubsection{Application of Tutte's factor theorem}

Theorem 1.3 .15 will be proved by applying not only Theorem 2.4 .5 but also some $f$-factor lemmas (such as, Lemma 2.4.10) in this section.

Definition 2.4.7. Let $G$ be a graph and $f$ be an integer-valued function defined on $V(G)$. An $f$-factor of $G$ is a subgraph $H$ of $G$ such that $\operatorname{deg}_{H}(v)=f(v)$ for each vertex $v$ of $G$. In particular, if the range of $f$ is $\{1,2\}$, we simply call $H$ an $\{1,2\}$-factor. 
In [48], Tutte gave an necessary and sufficient condition of the existence of $f$-factors.

Theorem 2.4.8 (Tutte [48]). A graph $G$ has an $f$-factor if and only if for any two vertex subsets $S, T$ of $V(G)$ with $S \cap T=\emptyset$,

$$
\sum_{v \in S} f(v) \geq|\mathcal{O}(S, T)|+\sum_{v \in T}\left(f(v)-\operatorname{deg}_{G-S}(v)\right),
$$

where $\mathcal{O}(S, T)$ is the set of components of $G-S-T$ for which

$$
\sum_{v \in U} f(v)+e(U, T) \equiv 1 \quad(\bmod 2) .
$$

Next we apply Tutte's $f$-factor theorem to find an $\{1,2\}$-factor for graphs given below.

Lemma 2.4.9. Let $\lambda_{o}$ be an odd integer and $G$ be an odd- $\lambda_{o}$-edge-connected graph. Let $\left\{V_{1}, V_{2}\right\}$ be a partition of $V(G)$ such that $\operatorname{deg}_{G}(v)=\lambda_{o}$ if $v \in V_{1}$ and $\operatorname{deg}_{G}(v)=2 \lambda_{o}$ if $v \in V_{2}$. If $f$ is a function defined on $V(G)$ such that $f(v)=\operatorname{deg}_{G}(v) / \lambda_{o}$ for each vertex $v$ of $G$, then $G$ has an $f$-factor.

Proof. Let $S$ and $T$ be two disjoint vertex subsets of $G$ and $\mathcal{O}=\mathcal{O}(S, T)$. Let $\left\{Q_{1}, Q_{2}, Q_{3}, Q_{4}\right\}$ be a partition of $T$, where for each $t \in\{1,2\}, Q_{t}$ consists of the vertices $v \in T \cap V_{t}$ such that $d_{G-S}(v)=0, Q_{3}$ consists of the vertices $v$ of $T \cap V_{2}$ such that $d_{G-S}(v)=1$, and $Q_{4}=T-Q_{1}-Q_{2}-Q_{3}$. The following claim directly follows from the definitions.

Claim 5. (1) $\lambda_{o} f(v)=d_{G}(v)$ and $f(v) \equiv d_{G}(v)(\bmod 2)$ for each vertex $v$.

(2) $\sum_{v \in U} d_{G}(v)+e(U, T) \equiv 1(\bmod 2)$ for each $U \in \mathcal{O}$.

Now we partition $\mathcal{O}$ into $\mathcal{O}_{1}$ and $\mathcal{O}_{2}$, where $\mathcal{O}_{1}=\{U \in \mathcal{O}: e(U, T)=0\}$ and $\mathcal{O}_{2}=\{U \in \mathcal{O}: \quad e(U, T) \neq 0\}$

Claim 6. $e(U, S) \geq \lambda_{o}$ if $U \in \mathcal{O}_{1}$ and $e(U, S) \geq 1$ if $U \in \mathcal{O}_{2}$. Therefore

$$
\sum_{U \in \mathcal{O}} e(U, S) \geq \lambda_{o}\left|\mathcal{O}_{1}\right|+\left|\mathcal{O}_{2}\right| .
$$


Proof. Note that if $U \in \mathcal{O}_{1}$, then $e(U, T)=0$ and thus $E(U, S)$ is an edge-cut. Since $G$ is odd- $\lambda_{o}$-edge-connected, it suffices to show that $e(U, S) \equiv 1(\bmod 2)$ for each $U \in \mathcal{O}$.

For each $U \in \mathcal{O}$, we have $\sum_{v \in U} d_{G}(v) \equiv e(U, T)+e(U, S)(\bmod 2)$. Since $a \equiv-a$ $(\bmod 2)$ for every integer $a$, it follows from Claim $5-(2)$ that $e(U, S) \equiv 1(\bmod 2)$.

\section{Claim 7.}

$$
e(S, T)=\sum_{v \in T}\left[d_{G}(v)-d_{G-S}(v)\right] \geq \lambda_{o} \sum_{v \in T}\left[f(v)-d_{G-S}(v)\right]+\left(\lambda_{o}-1\right)\left|\mathcal{O}_{2}\right| .
$$

Proof. Since $d_{G-S}(v)=0$ if $v \in Q_{1} \cup Q_{2}$ and $d_{G-S}(v)=1$ if $v \in Q_{3}$, we have

$$
\sum_{v \in Q_{1} \cup Q_{2} \cup Q_{3}}\left[d_{G}(v)-d_{G-S}(v)\right]=\lambda_{o} \sum_{v \in Q_{1} \cup Q_{2} \cup Q_{3}}\left[f(v)-d_{G-S}(v)\right]+\left(\lambda_{o}-1\right) \sum_{v \in Q_{3}} d_{G-S}(v)
$$

Since $\lambda_{o} f(v)=d_{G}(v)$ for each vertex $v$, we have

$$
\begin{aligned}
\sum_{v \in Q_{4}}\left[d_{G}(v)-d_{G-S}(v)\right] & =\sum_{v \in Q_{4}}\left[\lambda_{o} f(v)-d_{G-S}(v)\right] \\
& =\lambda_{o} \sum_{v \in Q_{4}}\left[f(v)-d_{G-S}(v)\right]+\left(\lambda_{o}-1\right) \sum_{v \in Q_{4}} d_{G-S}(v) .
\end{aligned}
$$

Combining (2.5) and (2.6), we have

$$
\sum_{v \in T}\left[d_{G}(v)-d_{G-S}(v)\right]=\lambda_{o} \sum_{v \in T}\left[f(v)-d_{G-S}(v)\right]+\left(\lambda_{o}-1\right) \sum_{v \in Q_{3} \cup Q_{4}} d_{G-S}(v) .
$$

Since each vertex $v \in Q_{3} \cup Q_{4}$ is adjacent to at most $d_{G-S}(v)$ components in $\mathcal{O}_{2}$, we have

$$
\sum_{v \in Q_{3} \cup Q_{4}} d_{G-S}(v) \geq\left|\mathcal{O}_{2}\right|
$$

Combining (2.7) and (2.8), we have

$$
e(S, T) \geq \lambda_{o} \sum_{v \in T}\left[f(v)-d_{G-S}(v)\right]+\left(\lambda_{o}-1\right)\left|\mathcal{O}_{2}\right| .
$$


Denote $S^{c}=V(G)-S$. Now we are to estimate $e\left(S, S^{\mathrm{c}}\right)$ in two ways by finding a lower bound and upper bound. Obviously,

$$
e\left(S, S^{c}\right) \leq \sum_{v \in S} d_{G}(v)=\lambda_{o} \sum_{v \in S} f(v)
$$

On the other hand,

$$
e\left(S, S^{c}\right) \geq e(S, T)+\sum_{U \in \mathcal{O}} e(S, U)
$$

By (2.9) and (2.10) together with Claim 6 and 7, we have

$$
\begin{aligned}
\lambda_{o} \sum_{v \in S} f(v) & \geq \lambda_{o} \sum_{v \in T}\left[f(v)-d_{G-S}(v)\right]+\left(\lambda_{o}-1\right)\left|\mathcal{O}_{2}\right|+\lambda_{o}\left|\mathcal{O}_{1}\right|+\left|\mathcal{O}_{2}\right| \\
& =\lambda_{o} \sum_{v \in T}\left[f(v)-d_{G-S}(v)\right]+\lambda_{o}\left(\left|\mathcal{O}_{1}\right|+\left|\mathcal{O}_{2}\right|\right) \\
& =\lambda_{o}\left(\sum_{v \in T}\left[f(v)-d_{G-S}(v)\right]+|\mathcal{O}|\right) .
\end{aligned}
$$

By (2.11), we have

$$
\sum_{v \in S} f(v) \geq|\mathcal{O}|+\sum_{v \in T}\left[f(v)-d_{G-S}(v)\right]
$$

Therefore, by Theorem 2.4.8, $G$ has an $f$-factor.

Lemma 2.4.10. Let $G$ be a graph with odd-edge-connectivity at least $(2 p+1)$. If there is a mapping $\mu: V(G) \rightarrow \mathbb{Z}^{+}$such that, for each vertex $v$ of $G, \operatorname{deg}_{G}(v)=(2 p+1) \mu(v)$, then there is a spanning subgraph $F$ of $G$ such that $\operatorname{deg}_{F}(v)=p \mu(v)$.

Proof. For each vertex $v$ of $G$ with $\operatorname{deg}_{G}(v) \notin\{2 p+1,2(2 p+1)\}$, we first apply Corollary 2.4.6 to $v$ with $a=2(2 p+1)$ and $\lambda_{o}=2 p+1$. Repeat this process until the degree of every vertex is either $(2 p+1)$ or $2(2 p+1)$. Let $G^{\prime}$ be the resulting graph.

Now we apply Lemma 2.4.9 to $G^{\prime}$ with $\lambda_{o}=2 p+1$. Let $F_{0}$ be a $(1,2)$-factor of $G^{\prime}$ such that for each vertex $v$ of $G^{\prime}, \operatorname{deg}_{F_{0}}(v)=1$ if $\operatorname{deg}_{G^{\prime}}(v)=2 p+1$ and $\operatorname{deg}_{F_{0}}(v)=2$ if $\operatorname{deg}_{G^{\prime}}(v)=2(2 p+1)$. 
Let $G^{\prime \prime}=G^{\prime}-E\left(F_{0}\right)$. Next we split each vertex $v$ of $G^{\prime \prime}$ with $\operatorname{deg}_{G^{\prime \prime}}(v)=4 p$ into a pair of degree $2 p$ vertices (no need to preserve the odd-edge-connectivity here). Let $G^{\prime \prime \prime}$ be the resulting $2 p$-regular graph. By Petersen theorem, $G^{\prime \prime \prime}$ has a 2 -factorization $\left\{F_{1}, \ldots, F_{p}\right\}$.

When $p$ is even, say $p=2 s$, the subgraph $F$ of $G$ induced by edges of $F_{1}, \ldots, F_{s}$ is a desired spanning subgraph. When $p$ is odd, say $p=2 s+1$, the subgraph $F$ of $G$ induced by edges of $F_{0}, F_{1}, \ldots, F_{s}$ is a desired spanning subgraph.

\subsubsection{Completion of the Proof of Theorem 1.3.15}

The proof of necessity is identical to that in [21]. Therefore, we only need to prove its sufficiency. Assume that $(G, \sigma)$ admits a modulo circular $\left(2+\frac{1}{p}\right)$-flow. By the equivalency between (B) and (C) in Theorem 1.3.11, we can further assume that $\tau$ itself is a modulo $(2 p+1)$-orientation of $(G, \sigma)$.

For each vertex $v \in V(G)$, let $H_{\tau}^{+}(v)=\{h \in H(v): \tau(h)=1\}$ and $H_{\tau}^{-}(v)=$ $\{h \in H(v): \tau(h)=-1\}$, and denote $\operatorname{deg}_{\tau}^{+}(v)=\left|H_{\tau}^{+}(v)\right|$ and $\operatorname{deg}_{\tau}^{-}(v)=\left|H_{\tau}^{-}(v)\right|$. If $\operatorname{deg}_{\tau}^{+}(v), \operatorname{deg}_{\tau}^{-}(v)>0$ for some vertex $v$, then apply Theorem 2.4.5 with $S(v)=$ $\left\{\left(e_{1}, e_{2}\right): e_{1} \in H_{\tau}^{+}(v)\right.$ and $\left.e_{2} \in H_{\tau}^{-}(v)\right\}$ and one can split a pair of half-edges (one from $H_{\tau}^{+}(v)$ and the other from $\left.H_{\tau}^{-}(v)\right)$ away from $v$. Let $G^{\prime}$ be the new graph obtained from $G$ after all possible splittings. Thus, in $G^{\prime},(2 p+1)$ is a divisor of each $\operatorname{deg}_{G^{\prime}}(v)$ and either $\operatorname{deg}_{\tau}^{+}(v)=0$ or $\operatorname{deg}_{\tau}^{-}(v)=0$ for each vertex $v$ of $G^{\prime}$, and, $G^{\prime}$ remains odd- $(2 p+1)$ edge-connected.

Applying Lemma 2.4.10 to the graph $G^{\prime}$, let $F$ be the spanning subgraph of $G^{\prime}$ such that $\operatorname{deg}_{F}(v)=p \mu(v)$ if $\operatorname{deg}_{G^{\prime}}(v)=(2 p+1) \mu(v)$ where $\mu$ is a mapping of $G^{\prime}: V\left(G^{\prime}\right) \rightarrow \mathbb{Z}^{+}$. Then $f^{*}$ is an integer-valued circular $\left(2+\frac{1}{p}\right)$-flow where

$$
f^{*}(e)= \begin{cases}p & \text { if } e \notin F \\ -p-1 & \text { if } e \in F\end{cases}
$$




\section{Chapter 3}

\section{Shortest Circuit Covers of Signed Graphs}

\subsection{Notation and Terminology}

Definition 3.1.1. Let $\mathcal{F}$ be a family of signed circuits of a signed graph $G$ and $K$ be a set of some nonnegative integers.

(1) $\mathcal{F}$ is a signed circuit cover (resp. signed circuit $K$-cover) of $G$ if each edge $e$ of $G$ belongs to $k_{e}$ members of $\mathcal{F}$ such that $k_{e} \geq 1$ (resp. $k_{e} \in K$ ). In particular, $\mathcal{F}$ is a signed circuit double cover of $G$ if $K=\{2\}$.

(2) The length of $\mathcal{F}$, denoted $\ell(\mathcal{F})$, is the total length of signed circuits in $\mathcal{F}$.

(3) A signed circuit cover $\mathcal{F}$ is a shortest circuit cover of $G$ if its length $\ell(\mathcal{F})$ is minimized. Furthermore, we denote by $\operatorname{SCC}(G)=\ell(\mathcal{F})$. 


\subsection{Lemmas and outline of the proofs}

Definition 3.2.1. Let $b$ be a bridge of a connected signed graph $G$ and $Q_{1}, Q_{2}$ be the two components of $G-\{b\}$. The bridge $b$ is an $g$-bridge of $G$ if $\epsilon_{N}\left(Q_{1}\right) \equiv \epsilon_{N}\left(Q_{2}\right) \equiv 0$ (mod 2). A signed graph $G$ is $g$-bridgeless if it contains no $g$-bridges.

Theorem 1.4.7-(2) can be revised as follows in a slightly stronger version.

Theorem 3.2.2. Let $G$ be an s-bridgeless signed graph with negativeness $\epsilon_{N}>0$.

(1) In general, we have

$$
\operatorname{SCC}(G) \leq|E|+3|V|+\min \left\{\frac{2}{3}|E|+\frac{4}{3} \epsilon_{N}-7,|V|+2 \epsilon_{N}-8\right\} .
$$

(2) If $G$ is g-bridgeless and $\epsilon_{N}$ is even, then

$$
\operatorname{SCC}(G) \leq|E|+2|V|+\min \left\{\frac{2}{3}|E|+\frac{1}{3} \epsilon_{N}-4,|V|+\epsilon_{N}-5\right\} .
$$

The following is the major lemma for the proof of Theorem 3.2.2.

Lemma 3.2.3. Let $G$ be an s-bridgeless signed graph with $\left|E_{N}(G)\right|=\epsilon_{N}(G)$. Then $G$ has a pair of subgraphs $\left\{G_{1}, G_{2}\right\}$ such that

(1) $E\left(G_{1}\right) \cup E\left(G_{2}\right)=E(G)$,

(2) $G_{1}$ contains no negative edge and is bridgeless, and

(3) $G_{2}-E_{N}(G)$ is acyclic and $G_{2}$ has a signed circuit $\{1,2, \ldots, k\}$-cover, where $k=2$ if $G$ is $g$-bridgeless with an even negativeness, and $k=3$ otherwise.

Lemma 3.2.3 will be proved in Section 3.4 after some preparations in Section 3.3. The main result, Theorem 3.2.2, will be proved as a corollary of Lemma 3.2.3 in Section 3.5. The following is the outline of the proof. By (1) of Lemma 3.2.3,

$$
\operatorname{SCC}(G) \leq \operatorname{SCC}\left(G_{1}\right)+\operatorname{SCC}\left(G_{2}\right)
$$

Lemma 3.2.3-(3) provides an estimation for $\operatorname{SCC}\left(G_{2}\right)$. For the bridgeless unsigned subgraph $G_{1}$, we use the classical results in Theorem 1.4.3 and 1.4.5. 


\subsection{Signed circuit covers of generalized barbells}

In this section, we study signed circuit covers of generalized barbells which play an important role in the proof of Lemma 3.2.3.

Recall that a graph is eulerian if it is connected and each vertex is of even degree. A vertex $v$ is a $k$-vertex of a graph $G$ if $v \in V_{k}(G)$.

Definition 3.3.1. A signed graph $H$ is a generalized barbell if it contains a set of vertex-disjoint eulerian subgraphs $\mathcal{B}=\left\{B_{1}, \ldots, B_{t}\right\}$ such that

(1) the contracted graph $X=H /\left(\cup_{i=1}^{t} B_{i}\right)$ is acyclic;

(2) for each vertex $x$ of $X,\left|E_{N}\left(B_{x}\right)\right| \equiv\left|E_{H}\left(V\left(B_{x}\right)\right)\right|(\bmod 2)$, where $B_{x}$ is the corresponding eulerian subgraph of $\mathcal{B}$ if $x$ is a contracted vertex and $E\left(B_{x}\right)=\emptyset$ otherwise.

We first study signed eulerian graphs with even number of negative edges which is a special case of generalized barbells.

Let $T$ be a closed eulerian trail of a signed eulerian graph. For any two vertices $u$ and $v$ of $T$, we use $u T v$ to denote the subsequence of $T$ starting with $u$ and ending with $v$ in the cyclic ordering induced by $T$.

Lemma 3.3.2. Every signed eulerian graph with even number of negative edges has a signed circuit double cover.

Proof. Let $B$ be a counterexample to Lemma 3.3.2 with $|E(B)|$ minimum. Then the maximum degree of $B$ is at least 4 otherwise $B$ is a balanced circuit. By the minimality of $B, B$ cannot be decomposed into two signed eulerian subgraphs, each of which contains an even number of negative edges. Thus we have the following observation

Observation. For any eulerian trail $T=u_{1} e_{1} u_{2} e_{2} \cdots u_{m} e_{m} u_{1}$ of $B$ where $m=|E(G)|$ and for any two integers $i, j \in[1, m]$ with $i<j$ and $u_{i}=u_{j}, u_{i} T u_{j}$ is a signed eulerian graph with odd number of negative edges. 
Pick an arbitrary eulerian trail $T=u_{1} e_{1} u_{2} e_{2} \cdots u_{m} e_{m} u_{1}$. We consider the following two cases.

Case 1. For any two integers $i \neq j \in[1, m]$, if $u_{i}=u_{j}$, then $|j-i| \equiv 1(\bmod m)$.

In this case, the resulting graph obtained from $B$ by deleting all loops is either a single vertex or a circuit. Since $B$ has an even number of negative edges, one can check that $B$ has a signed circuit double cover, a contradiction.

Case 2. There are two integers $i, j \in[1, m]$ such that $2 \leq j-i \leq m-2$ and $u_{i}=u_{j}$.

Let $B_{1}=u_{i} T u_{j}$ and $B_{2}=u_{j} T u_{i}$. Then, by Observation, both $B_{1}$ and $B_{2}$ are signed eulerian subgraphs of $B$ with $B=B_{1} \cup B_{2}$ such that $\left|E\left(B_{k}\right)\right| \geq 2$ and $\left|E_{N}\left(B_{k}\right)\right| \equiv 1$ $(\bmod 2)$ for each $k=1,2$.

If $V\left(B_{1}\right) \cap V\left(B_{2}\right)=\left\{u_{i}\right\}$, then for each $k=1,2$, let $B_{k}^{\prime}$ be the resulting graph obtained from $B_{k}$ by adding a negative loop $e_{k}^{\prime}$ at $u_{i}$. Clearly, $B_{k}^{\prime}$ remains eulerian, $\left|E\left(B_{k}^{\prime}\right)\right|<|E(B)|$, and $\left|E_{N}\left(B_{k}^{\prime}\right)\right|$ is even. By the minimality of $B, B_{k}^{\prime}$ has a signed circuit double cover $\mathcal{F}_{k}$. Since $e_{k}^{\prime}$ is a negative loop of $B_{k}^{\prime}$, it is covered by two barbells, say $C_{k}^{1}$ and $C_{k}^{2}$, in $\mathcal{F}_{k}$. Let $C^{\ell}=\cup_{k=1}^{2}\left(C_{k}^{\ell}-e_{k}^{\prime}\right)$ for each $\ell=1,2$. Since $V\left(B_{1}\right) \cap V\left(B_{2}\right)=$ $\left\{u_{i}\right\}$, both $C^{1}$ and $C^{2}$ are two barbells of $B$, and so $B$ has a signed circuit double cover $\cup_{k=1}^{2}\left(\mathcal{F}_{k}-\left\{C_{k}^{1}, C_{k}^{2}\right\}\right) \cup\left\{C^{1}, C^{2}\right\}$, a contradiction.

If $V\left(B_{1}\right) \cap V\left(B_{2}\right) \neq\left\{u_{i}\right\}$, then there are two integers $s$ and $t$ such that $s \in[i, j]$, $t \notin[i, j]$, and $u_{s}=u_{t}$. By Observation, $\left|E_{N}\left(u_{s} T u_{t}\right)\right| \equiv 1(\bmod 2)$. Let $T^{*}$ be a new closed eulerian trail of $B$ obtained from $T$ by reversing the subsequence $u_{i} T u_{j}$ in $T$. Then $E\left(u_{s} T^{*} u_{t}\right)$ is the disjoint union of $E\left(u_{i} T u_{s}\right)$ and $E\left(u_{j} T u_{t}\right)$ and thus $E_{N}\left(u_{s} T^{*} u_{t}\right)$ is the disjoint union of $E_{N}\left(u_{i} T u_{s}\right)$ and $E_{N}\left(u_{j} T u_{t}\right)$. Since $\left|E_{N}\left(u_{i} T u_{j}\right)\right| \equiv 1(\bmod 2)$ and $\left.\mid E_{N}\left(u_{s} T u_{t}\right)\right) \mid \equiv 1(\bmod 2)$, we have $\left|E_{N}\left(u_{i} T u_{s}\right)\right| \equiv\left|E_{N}\left(u_{j} T u_{t}\right)\right|(\bmod 2)$. Therefore $\left|E_{N}\left(u_{s} T^{*} u_{t}\right)\right| \equiv 0(\bmod 2)$, a contradiction to Observation. This completes the proof of the lemma.

The following lemma is a generalization of Lemma 3.3.2.

Lemma 3.3.3. Every generalized barbell $H$ has a signed circuit double cover. 
Proof. Let $\left\{B_{1}, \ldots, B_{t}\right\}$ be a set of vertex-disjoint eulerian subgraphs of $H$ and $X=$ $H /\left(\cup_{i=1}^{t} B_{i}\right)$ as described in Definition 3.3.1. We proceed the proof by induction on $\left|E(H)-\cup_{i=1}^{t} E\left(B_{i}\right)\right|$.

If $E(H)-\cup_{i=1}^{t} E\left(B_{i}\right)=\emptyset$, then by the definition of generalized barbell, each component of $H$ is a signed eulerian graph with an even number of negative edges. Thus $H$ has a signed circuit double cover by Lemma 3.3.2.

Now assume that $E(H)-\cup_{i=1}^{t} E\left(B_{i}\right) \neq \emptyset$. Let $u v \in E(H)-\cup_{i=1}^{t} E\left(B_{i}\right)$ and $H^{\prime}$ be the new signed graph obtained from $H$ by deleting $u v$ and adding negative loops $e_{u}$ and $e_{v}$ at $u$ and $v$, respectively. By the definition, $H^{\prime}$ remains as a generalized barbell. Since $X$ is acyclic, $H^{\prime}$ has more components than $H$, and thus by induction to each component of $H^{\prime}, H^{\prime}$ has a signed circuit double cover $\mathcal{F}^{\prime}$. Let $\left\{C_{u}^{1}, C_{u}^{2}\right\}$ and $\left\{C_{v}^{1}, C_{v}^{2}\right\}$ be the sets of barbells in $\mathcal{F}^{\prime}$ containing $e_{u}$ and $e_{v}$, respectively. Since $e_{u}$ and $e_{v}$ belong to two distinct components of $H^{\prime}, C^{i}=\left(C_{u}^{i}-e_{u}\right) \cup\left(C_{v}^{i}-e_{v}\right)+u v(i=1,2)$ is a barbell in $H$. Hence

$$
\left(\mathcal{F}^{\prime}-\left\{C_{u}^{1}, C_{u}^{2}, C_{v}^{1}, C_{v}^{2}\right\}\right) \cup\left\{C^{1}, C^{2}\right\}
$$

is a signed circuit double cover of $H$.

Lemma 3.3.4. Let $H$ be a generalized barbell with a set of vertex-disjoint eulerian subgraphs $\mathcal{B}=\left\{B_{1}, \ldots, B_{t}\right\}$, and assume that $\left\{B_{1}, \ldots, B_{s}\right\}(2 \leq s \leq t)$ is the set of eulerian subgraphs corresponding to the 1-vertices of the contracted graph $X=H /\left(\cup_{i=1}^{t} B_{i}\right)$. If each $B_{i}(1 \leq i \leq t)$ is a circuit, then there is a family of signed circuits $\mathcal{F}$ in $H$ such that each edge e of $H$ belongs to

(a) exactly one member of $\mathcal{F}$ if $e \in \cup_{i=1}^{s} E\left(B_{i}\right)$,

(b) one or two members of $\mathcal{F}$ if $e \in \cup_{i=s+1}^{t} E\left(B_{t}\right)$, and

(c) at most one member of $\mathcal{F}$ if $e \in E(H)-\cup_{i=1}^{t} E\left(B_{i}\right)$.

Proof. Assume that $H$ is embedded in the plane and let $\overline{X^{*}}$ be a graph obtained from $X$ by first clockwise splitting each vertex $x$ with even degree into $\frac{1}{2} \operatorname{deg}_{X}(x)$ 2-vertices, and replacing each maximal subdivided edge with a single edge. Then each vertex of $\overline{X^{*}}$ is of 
odd degree. By the definition of generalized barbell, $\overline{X^{*}}$ is a forest and $V\left(\overline{X^{*}}\right)$ corresponds to the set of unbalanced circuits of $\mathcal{B}$. Thus $\overline{X^{*}}$ has a spanning subgraph satisfying that each component is a star graph with at least two vertices. Let $K_{1, r_{i}}(i=1, \ldots, \ell)$ be all such star subgraphs.

Note that $V\left(\overline{X^{*}}\right)=\cup_{i=1}^{\ell} V\left(K_{1, r_{i}}\right)$ corresponds to the set of unbalanced circuits of $\mathcal{B}$. For $1 \leq i \leq \ell$, one can check that the subgraph of $H$ corresponding to $K_{1, r_{i}}$ has a signed circuit cover $\mathcal{F}_{i}$ such that each edge of the unbalanced circuits corresponding to 1-vertices of $K_{1, r_{i}}$ is covered by $\mathcal{F}_{i}$ exactly once and each edge of the unbalanced circuit corresponding to the unique vertex of $K_{1, r_{i}}$ with degree $r_{i} \geq 2$ is covered by $\mathcal{F}_{i}$ once or twice. Therefore the union of $\cup_{i=1}^{\ell} \mathcal{F}_{i}$ together with the set of balanced circuits of $\mathcal{B}$ is a desired family $\mathcal{F}$ of signed circuits of $H$.

The following result is stronger than Lemma 3.3.3 which states that a generalized barbell has a signed circuit $\{1,2\}$-cover with some edges covered only once.

Lemma 3.3.5. For each generalized barbell, it either

(1) can be decomposed into balanced circuits, or

(2) has a signed circuit $\{1,2\}$-cover $\mathcal{F}$ such that there are two edge-disjoint unbalanced circuits $C_{1}$ and $C_{2}$ whose edges are covered by $\mathcal{F}$ exactly once.

Proof. Let $H$ be a counterexample to Lemma 3.3.5 with $|E(H)|$ minimum. Thus $H$ is connected. Otherwise each component of $H$ satisfies either (1) or (2). This implies that $H$ satisfies either (1) or (2), a contradiction to the choice of $H$.

Claim 8. $H$ is eulerian and therefore contains an even number of negative edges.

Proof. By the definition of generalized barbell, it is sufficient to show that $H$ is bridgeless. Suppose to the contrary that $H$ has a bridge. By Lemma 3.3.3, $H$ has a signed circuit double cover $\mathcal{F}^{\prime}$. Since $H$ has bridges, $\mathcal{F}^{\prime}$ contains a barbell $C$ with two unbalanced circuits $C_{1}$ and $C_{2}$. Then $\mathcal{F}=\mathcal{F}^{\prime}-\{C\}$ is a signed circuit $\{1,2\}$-cover of $H$ and covers $C_{1}$ and $C_{2}$ exactly once, a contradiction. This proves the claim. 
Since $H$ is eulerian by Claim $8, H$ has a decomposition

$$
\mathcal{C}=\left\{C_{1}, \ldots, C_{h}, C_{h+1}, \ldots, C_{h+m}, C_{h+m+1}, \ldots, C_{h+m+n}\right\},
$$

where $h, m$ and $n$ are three nonnegative integers, and each $C_{i}$ is an unbalanced circuit if $1 \leq i \leq h$, a short barbell if $h+1 \leq i \leq h+m$, and a balanced circuit otherwise. We choose such a decomposition that

(a) $h+2 m+n$ is as large as possible,

(b) subject to (a), $n$ is as large as possible, and

(c) subject to (a) and (b), $m$ is as large as possible.

Claim 9. $h \geq 2$ is an even integer and $\left|V\left(C_{i}\right) \cap V\left(C_{j}\right)\right|=0$ for $1 \leq i<j \leq h$.

Proof. If $h=0$, then $\mathcal{C}$ satisfies (1) if $m=0$, and $\mathcal{C}-\left\{C_{1}\right\}$ satisfies (2) otherwise. Thus we can assume $h>0$. Since $\left|E_{N}(H)\right|=\sum_{i=1}^{h+m+n}\left|E_{N}\left(C_{i}\right)\right|$ is even and $\left|E_{N}\left(C_{i}\right)\right|$ is even for $h+1 \leq i \leq h+m+n$, we have that $h \geq 2$ is even.

Let $C_{i}$ and $C_{j}$ be two circuits in $\mathcal{C}$ with $1 \leq i<j \leq h$. If $\left|V\left(C_{i}\right) \cap V\left(C_{j}\right)\right| \geq 3$, then $C_{i} \cup C_{j}$ can be decomposed into three or more circuits (balanced or unbalanced), a contradiction to (a). Thus, $\left|V\left(C_{i}\right) \cap V\left(C_{j}\right)\right| \leq 2$. If $\left|V\left(C_{i}\right) \cap V\left(C_{j}\right)\right|=2$, then $C_{i} \cup C_{j}$ has a decomposition into two balanced circuits since both $C_{i}$ and $C_{j}$ are unbalanced circuits, which contradicts (b). If $\left|V\left(C_{i}\right) \cap V\left(C_{j}\right)\right|=1$, then $C_{i} \cup C_{j}$ is a short barbell, which contradicts (c). Thus, the claim is true.

Let $H^{\prime}=H /\left(\cup_{i=1}^{h} C_{i}\right)$ and for $1 \leq i \leq h$, let $c_{i}$ be the vertex of $H^{\prime}$ corresponding to $C_{i}$. Let $T^{\prime}$ be a spanning tree of $H^{\prime}$ since $H$ is connected. By Claim $9, h \geq 2$ is even. Let $P_{j}\left(1 \leq j \leq \frac{h}{2}\right)$ be a path in $T^{\prime}$ from $c_{2 j-1}$ to $c_{2 j}$ and let

$$
F^{\prime}=T^{\prime}\left[\Delta_{j=1}^{\frac{h}{2}} E\left(P_{j}\right)\right]
$$

Then $F^{\prime}$ is a forest and $\left\{c_{1}, \ldots, c_{h}\right\}$ is the set of vertices of $F^{\prime}$ with odd degree. By the definition, the subgraph of $H$ corresponding to $F^{\prime}$ is a generalized barbell satisfying the conditions in Lemma 3.3.4, and thus, by Lemma 3.3.4, it has a family $\mathcal{F}^{*}$ of signed circuits 
such that $\mathcal{F}=\mathcal{F}^{*} \cup\left\{C_{h+1}, \ldots, C_{h+m+n}\right\}$ is a signed circuit $\{1,2\}$-cover of $H$ and at least two unbalanced circuits in $\left\{C_{1}, \ldots, C_{h}\right\}$ are covered by $\mathcal{F}$ exactly once, a contradiction. This completes the proof of Lemma 3.3.5.

\subsection{Proof of Lemma 3.2.3}

In this section, we complete the proof of Lemma 3.2.3. For a signed graph $G$, we use $B(G)$ to denote the set of bridges of $G$ and for each $e \in E_{N}(G)$, define

$$
S_{G}(e)=\{e\} \cup\{f:\{e, f\} \text { is a 2-edge-cut of } G\} .
$$

Let $B_{g}(G)$ be the subset of $B(G)$ such that, for each $b \in B_{g}(G)$, at least one component of $G-\{b\}$ contains an odd number of negative edges, and let $B_{s}(G)$ be the subset of $B(G)$ such that, for each $b \in B_{s}(G)$, each component of $G-\{b\}$ contains negative edges. We need the following lemmas.

Lemma 3.4.1. Let $H$ be a signed graph satisfying that $\left|E_{N}(H)\right| \geq 2$ and $H-E_{N}(H)$ is a spanning tree of $H$. If $\left|E_{N}(H)\right|$ is even, then $H$ has a generalized barbell containing all edges of $B_{g}(H) \cup\left(\cup_{e \in E_{N}(H)} S_{H}(e)\right)$.

Proof. Let $T=H-E_{N}(H)$. Then $E(H)$ is the disjoint union of $E(T)$ and $E_{N}(H)$. For each $e \in E_{N}(H)$, let $C_{e}$ be the unique circuit of $T+e$.

Let $H^{\prime}=\triangle_{e \in E_{N}(H)} C_{e}$ and $O_{H^{\prime}}$ be the set of all components of $H^{\prime}$ containing an odd number of negative edges. Since $\left|E_{N}(H)\right|$ is even, so is $\left|O_{H^{\prime}}\right|$. Let $\left\{v_{1}, v_{2}, \ldots, v_{2 t-1}, v_{2 t}\right\}$ be the set of vertices of the contracted graph $H / H^{\prime}$ corresponding to $O_{H^{\prime}}$. For each $1 \leq i \leq t$, there is a shortest path $P_{i}$ in $H / H^{\prime}$ from $v_{2 i-1}$ to $v_{2 i}$. Note that $E_{N}(H) \subseteq E\left(H^{\prime}\right)$ and hence $E\left(P_{i}\right) \subseteq E\left(H / H^{\prime}\right) \subseteq E(T)$. Since $T$ is a tree of $H, H^{\prime \prime}=H^{\prime} \cup\left(\triangle_{i=1}^{t} P_{i}\right)$ is a generalized barbell.

For every bridge $b \in B_{g}(H)$, each component of $H-\{b\}$ contains an odd number of negative edges since $\left|E_{N}(H)\right|$ is even, and thus contains an odd number of members of $O_{H^{\prime}}$. This fact implies that $b$ must belong to an odd number of members of $\left\{P_{1}, \ldots, P_{t}\right\}$ 
and thus $b \in E\left(H^{\prime \prime}\right)$. Hence $B_{g}(H) \subseteq E\left(H^{\prime \prime}\right)$. For every $e \in E_{N}(H)$, it is obvious that $S_{H}(e) \subseteq E\left(C_{e}\right)$ and $S_{H}(e) \cap E\left(C_{f}\right)=\emptyset$ for any $f \in E_{N}(G)-\{e\}$, which implies that $S_{H}(e) \subseteq E\left(H^{\prime}\right)$. Therefore, $\cup_{e \in E_{N}(H)} S_{H}(e) \subseteq E\left(H^{\prime}\right) \subseteq E\left(H^{\prime \prime}\right)$.

Lemma 3.4.2. Let $H$ be a signed graph satisfying that $\left|E_{N}(H)\right| \geq 2$ and $H-E_{N}(H)$ is a spanning tree of $H$. Then $H$ has a signed circuit $\{0,1,2,3\}$-cover such that each edge of $B_{s}(H) \cup\left(\cup_{e \in E_{N}(H)} S_{H}(e)\right)$ is covered at least once and each negative loop (if any) is covered precisely twice.

Proof. Let $H$ be a counterexample with $|E(H)|$ minimum.

Claim 10. $B(H)=\emptyset$.

Proof. Suppose to the contrary $B(H) \neq \emptyset$. Let $b=u_{1} u_{2} \in B(H)$ and $Q_{1}$ and $Q_{2}$ be the two components of $H-\{b\}$ such that $u_{i} \in Q_{i}$ for $i=1,2$.

If $b \in B(H)-B_{s}(H)$, then there is one member in $\left\{Q_{1}, Q_{2}\right\}$, without loss of generality, say $Q_{1}$, satisfying that $B_{s}\left(Q_{1}\right)=B_{s}(H)$ and $E_{N}\left(Q_{1}\right)=E_{N}(H)$. By the minimality of $H$, $Q_{1}$ (and thus $H$ ) has a desired signed circuit $\{0,1,2,3\}$-cover, a contradiction.

If $b \in B_{s}(H)$, then $\left|E_{N}\left(Q_{1}\right)\right| \geq 1$ and $\left|E_{N}\left(Q_{2}\right)\right| \geq 1$. For each $i=1,2$, let $Q_{i}^{*}$ be the graph obtained from $Q_{i}$ by adding a negative loop $e_{i}$ at $u_{i}$. It is easy to see that $B_{s}\left(Q_{1}^{*}\right) \cup B_{s}\left(Q_{2}^{*}\right)=B_{s}(H)-\{b\}$ and $\cup_{i=1}^{2}\left(E_{N}\left(Q_{i}^{*}\right)-\left\{e_{i}\right\}\right)=E_{N}(H)$. By the minimality of $H$, each $Q_{i}^{*}$ has a signed circuit $\{0,1,2,3\}$-cover $\mathcal{F}_{i}^{*}$ which covers each edge of $B_{s}\left(Q_{i}^{*}\right) \cup E_{N}\left(Q_{i}^{*}\right)$ at least once and covers each negative loop of $Q_{i}^{*}$ exactly twice. Let $C_{i}^{1}$ and $C_{i}^{2}$ be the two signed circuits in $\mathcal{F}_{i}^{*}$ containing $e_{i}$. Since $e_{i}$ is a negative loop, $C_{i}^{j}$ $(j=1,2)$ is a barbell of $Q_{i}^{*}$, and thus $C^{j}=\left(C_{1}^{j}-e_{1}\right) \cup\left(C_{2}^{j}-e_{2}\right)+b$ is also a barbell of $H$. Therefore, $\mathcal{F}=\left(\mathcal{F}_{1}^{*}-\left\{C_{1}^{1}, C_{1}^{2}\right\}\right) \cup\left(\mathcal{F}_{2}^{*}-\left\{C_{2}^{1}, C_{2}^{2}\right\}\right) \cup\left\{C^{1}, C^{2}\right\}$ is a desired signed circuit $\{0,1,2,3\}$-cover of $H$, a contradiction.

Claim 10 implies that $H$ is 2-edge-connected. Thus, Lemma 3.4.2 follows from Lemmas 3.4.1 and 3.3.3 if $\left|E_{N}(H)\right|$ is even. Since $\left|E_{N}(H)\right| \geq 2$, in the following context, we assume that $\left|E_{N}(H)\right| \geq 3$ is odd. 
Let $T=H-E_{N}(H)$. Note that $T$ is a spanning tree of $H$ and $E(H)$ is the disjoint union of $E(T)$ and $E_{N}(H)$. For each $e \in E_{N}(H)$, let $C_{e}$ be the unique circuit of $T+e$.

Claim 11. For every $e \in E_{N}(H), H$ has a signed circuit containing all edges of $S_{H}(e)$.

Proof. Let $e \in E_{N}(H)$ and $f \in E_{N}(H)-\{e\}$. Note that $S_{H}(e) \subseteq E\left(C_{e}\right), S_{H}(f) \subseteq E\left(C_{f}\right)$ and $S_{H}(e) \cap S_{H}(f)=\emptyset$ (it can be checked easily since $T=H-E_{N}(H)$ is a spanning tree of $H)$. If $\left|V\left(C_{e}\right) \cap V\left(C_{f}\right)\right| \leq 1$, then there is a shortest path $P$ in $T$ joining $C_{e}$ to $C_{f}$ (note that $P$ is a single vertex if $\left|V\left(C_{e}\right) \cap V\left(C_{f}\right)\right|=1$ ), and thus $C_{e} \cup C_{f} \cup P$ is a desired signed circuit. If $\left|V\left(C_{e}\right) \cap V\left(C_{f}\right)\right| \geq 2$, since $T$ is a spanning tree of $H$, then $C_{e} \cap C_{f}$ is a path containing no edges of $S_{H}(e)$. Thus $C_{e} \Delta C_{f}$ is a balanced circuit as desired.

Claim 12. Each edge $e \in E_{N}(H)$ is contained in a 2-edge-cut of $H$.

Proof. Suppose to the contrary that there is a negative edge $e \in E_{N}(H)$ such that $H_{0}=$ $H-\{e\}$ remains 2-edge-connected. If $H$ contains negative loops, we choose $e$ which is a negative loop.

Since $H_{0}$ is 2-edge-connected and $\left|E_{N}\left(H_{0}\right)\right|=\left|E_{N}(H)-\{e\}\right| \geq 2$ is even, Lemma 3.4.1 implies that $H_{0}$ has a generalized barbell $H_{1}$ containing all edges of $\cup_{f \in E_{N}\left(H_{0}\right)} S_{H_{0}}(f)$. Let $\mathcal{F}_{1}$ be a signed circuit double cover of $H_{1}$ by Lemma 3.3.3. Note that $S_{H}(e)=\{e\}$ and $S_{H}(f) \subseteq S_{H_{0}}(f)$ for any $f \in E_{N}\left(H_{0}\right)=E_{N}(H)-\{e\}$. Thus $\cup_{f \in E_{N}(G)} S_{H}(f) \subseteq$ $\{e\} \cup\left(\cup_{f \in E_{N}\left(H_{0}\right)} S_{H_{0}}(f)\right)$.

If $e$ is not a negative loop of $H$, then $H$ has no loop, but has a signed circuit $C$ containing $e$ by Claim 11. Thus $\mathcal{F}=\mathcal{F}_{1} \cup\{C\}$ is a signed circuit $\{0,1,2,3\}$-cover of $H$ covering all edges of $\cup_{f \in E_{N}(H)} S_{H}(f)$, a contradiction. Assume that $e$ is a negative loop of $G$ and let $u$ denote the unique endvertex of $e$.

If $\mathcal{F}_{1}$ contains a barbell $C$, then let $C_{1}$ and $C_{2}$ be the two unbalanced circuits of $C$. Since $H$ is 2-edge-connected, there are two edge-disjoint paths in $H$ from $u$ to $C_{1}$ and $C_{2}$, denoted by $P_{1}$ and $P_{2}$, respectively. Then $C_{i}^{\prime}=C_{i} \cup P_{i}+e_{0}$ for $i=1,2$ is a barbell of $H$. Since $\mathcal{F}_{1}$ is a signed circuit double cover of $H_{1}, \mathcal{F}=\left(\mathcal{F}_{1}-C\right) \cup\left\{C_{1}^{\prime}, C_{2}^{\prime}\right\}$ is a desired signed circuit $\{0,1,2,3\}$-cover of $H$, a contradiction. 
If $\mathcal{F}_{1}$ contains no barbells, then $e$ is the unique loop of $H$. Note that $H_{1}$ is a generalized barbell. By Lemma 3.3.5, $H_{1}$ has either a decomposition $\mathcal{F}_{1}^{\prime}$ into balanced circuits or a signed circuit $\{1,2\}$-cover $\mathcal{F}_{1}^{\prime \prime}$ and two edge-disjoint unbalanced circuit $C_{1}$ and $C_{2}$ such that each edge in $E\left(C_{1}\right) \cup E\left(C_{2}\right)$ is covered by $\mathcal{F}_{1}^{\prime \prime}$ exactly once. In the former case, let $C^{\prime}$ be a signed circuit containing $e$ by Claim 11. Then the family $\mathcal{F}=\mathcal{F}_{1}^{\prime} \cup\left\{C^{\prime}, C^{\prime}\right\}$ is a desired signed circuit $\{0,1,2,3\}$-cover of $H$. In the latter case, since $H$ is 2-edgeconnected, there are two edge-disjoint paths of $H$ from $u$ to $C_{1}$ and $C_{2}$, denoted by $P_{1}$ and $P_{2}$, respectively. Similar to the case when $\mathcal{F}_{1}$ contains a barbell, we can construct a desired signed circuit $\{0,1,2,3\}$-cover of $H$, and thus obtain a contradiction.

By Claim 12, $H$ contains no negative loops and $\left|S_{H}(e)\right| \geq 2$ for each $e \in E_{N}(H)$. For every $e \in E_{N}(G)$, let $\mathcal{M}_{e}$ denote the set of all components of the subgraph $H-S_{H}(e)$.

Claim 13. For any e, $e^{\prime} \in E_{N}(H), S_{H}\left(e^{\prime}\right)$ is contained in exactly one member of $\mathcal{M}_{e}$.

Proof. Note that each member of $\mathcal{M}_{e}$ is 2-edge-connected, and $S_{H}(e) \cap S_{H}\left(e^{\prime}\right)=\emptyset$ since $H-E_{N}(H)$ is a spanning tree of $H$. Then $S_{H}\left(e^{\prime}\right) \subseteq \cup_{M \in \mathcal{M}_{e}} E(M)$. Let $e^{*}$ be an arbitrary edge in $S_{H}\left(e^{\prime}\right)-\left\{e^{\prime}\right\}$. If there are two distinct members $M_{i}$ and $M_{j}$ of $\mathcal{M}_{e}$ such that $e^{\prime} \in E\left(M_{i}\right)$ and $e^{*} \in E\left(M_{j}\right)$, then both $M_{i}-e^{\prime}$ and $M_{j}-e^{*}$ are connected, and thus $H-\left\{e^{\prime}, e^{*}\right\}$ is also connected. This contradicts that $\left\{e^{\prime}, e^{*}\right\}$ is a 2-edge-cut of $H$. Thus, $e^{\prime}$ and $e^{*}$ are contained in a common member of $\mathcal{M}_{e}$. The arbitrariness of $e^{*}$ implies that the claim holds.

For every $e \in E_{N}(H)$, let $m_{e}=\max \left\{\left|E_{N}(H) \cap E(M)\right|: M \in \mathcal{M}_{e}\right\}$. It is obvious that $m_{e} \leq\left|E_{N}(H)\right|-1$ since $e \notin \cup_{M \in \mathcal{M}_{e}} E(M)$.

Claim 14. $\max \left\{m_{e}: \quad e \in E_{N}(H)\right\}=\left|E_{N}(H)\right|-1$.

Proof. Let $e_{0} \in E_{N}(H)$ and $M_{01} \in \mathcal{M}_{e_{0}}$ such that

$$
m_{e_{0}}=\left|E_{N}(H) \cap E\left(M_{01}\right)\right|=\max \left\{m_{e}: \quad e \in E_{N}(H)\right\} .
$$

Suppose that $m_{e_{0}}<\left|E_{N}(H)\right|-1$. Then there is a member $M_{02} \in \mathcal{M}_{e_{0}}-\left\{M_{01}\right\}$ such that $M_{02}$ contains a negative edge $e_{1}$ of $H$. 
By Claim 13, $S_{H}\left(e_{1}\right) \subseteq E\left(M_{02}\right)$ and there is a member $M_{11} \in \mathcal{M}_{e_{1}}$ such that $S_{H}\left(e_{0}\right) \subseteq$ $E\left(M_{11}\right)$. Thus,

$$
\left\{e_{0}\right\} \cup E\left(M_{01}\right) \subseteq S_{H}\left(e_{0}\right) \cup\left(\cup_{M \in \mathcal{M}_{e_{0}}-\left\{M_{02}\right\}} E(M)\right) \subseteq E\left(M_{11}\right),
$$

which implies that

$$
m_{e_{1}} \geq\left|E_{N}(H) \cap E\left(M_{11}\right)\right| \geq 1+\left|E_{N}(H) \cap E\left(M_{01}\right)\right|=1+m_{e_{0}} .
$$

This contradicts the choice of $e_{0}$, and thus the claim holds.

By Claim 14, there is an edge $e \in E_{N}(H)$ such that $E_{N}(H)-\{e\}$ is contained in exactly one member of $\mathcal{M}_{e}$. Let $\mathcal{M}_{e}=\left\{M_{1}^{\prime}, \ldots, M_{s}^{\prime}\right\}$. Without loss of generality, assume that $E_{N}(H)-\{e\} \subseteq E\left(M_{1}^{\prime}\right)$ and all edges of $M_{i}^{\prime}(i=2, \ldots, s)$ are positive. Since $H$ is 2-edge-connected, it follows from the definition of $S_{H}(e)$ that $H / \cup_{i=1}^{s} M_{i}^{\prime}$ is a circuit, and each $M_{i}^{\prime}$ is also 2-edge-connected. Since $\left|E_{N}\left(M_{1}^{\prime}\right)\right|=\left|E_{N}(H)\right|-1 \geq 2$ is even, $M_{1}^{\prime}$ has a generalized barbell $H_{1}^{\prime}$ containing all edges of $\cup_{f \in E_{N}\left(M_{1}^{\prime}\right)} S_{M_{1}^{\prime}}(f)$ by Lemma 3.4.1, and $H_{1}^{\prime}$ has a signed circuit double cover $\mathcal{F}_{1}$ by Lemma 3.3.3.

Since $E_{N}\left(M_{1}^{\prime}\right)=E_{N}(H)-\{e\}$ and $S_{M_{1}^{\prime}}(f) \supseteq S_{H}(f)$ for any $f \in E_{N}\left(M_{1}^{\prime}\right)$,

$$
\cup_{f \in E_{N}(H)} S_{H}(f) \subseteq S_{H}(e) \cup\left(\cup_{f \in E_{N}\left(M_{1}^{\prime}\right)} S_{M_{1}^{\prime}}(f)\right) .
$$

By Claim 11, $H$ has a signed circuit $C$ containing all edges of $S_{H}(e)$, and thus $\mathcal{F}=\mathcal{F}_{1} \cup\{C\}$ is a desired signed circuit $\{0,1,2,3\}$-cover of $H$, a contradiction. This complete the proof of the lemma.

Lemma 3.4.3 (see [33]). Let $G$ be a signed graph. Then $\left|E_{N}(G)\right|=\epsilon_{N}(G)$ if and only if for every edge cut $T$ of $G,\left|E_{N}(G) \cap T\right| \leq \frac{|T|}{2}$.

Proof. Let $G$ be an s-bridgeless signed graph with $\left|E_{N}(G)\right|=\epsilon_{N}(G)$. Without loss of generality, we further assume that $G$ is connected. Since $G$ is s-bridgeless, we have $\left|E_{N}(G)\right| \neq 1$. If $\left|E_{N}(G)\right|=0$, then $G$ is a 2-edge-connected unsigned graph. The lemma is trivial, and thus assume that $\left|E_{N}(G)\right| \geq 2$.

Denote $G_{1}=G-B(G)-\left(\cup_{e \in E_{N}(G)} S_{G}(e)\right)$. By the definitions of $B(G)$ and $S_{G}(e), G_{1}$ contains no negative edges of $G$ and is bridgeless. To construct $G_{2}$, let $H=T+E_{N}(G)$, 
where $T$ is a spanning tree of $G-E_{N}(G)$ (the existence of $T$ is guaranteed by Lemma 3.4.3). We have the following observations:

(1) $E_{N}(G)=E_{N}(H)$;

(2) $B_{g}(G) \subseteq B_{g}(H)$;

(3) $B_{s}(G) \subseteq B_{s}(H)$;

(4) $S_{G}(e) \subseteq S_{H}(e)$ for each $e \in E_{N}(G)$.

By Lemma 3.4.2, $H$ has a signed circuit $\{0,1,2,3\}$-cover $\mathcal{F}_{2}$ such that each edge of $B_{s}(H) \cup\left(\cup_{e \in E_{N}(H)} S_{H}(e)\right)\left(\supseteq B_{s}(G) \cup\left(\cup_{e \in E_{N}(G)} S_{G}(e)\right)\right)$ is covered by $\mathcal{F}_{2}$ at least once. Let $G_{2}=G\left[\cup_{C \in \mathcal{F}_{2}} E(C)\right]$. Since $G$ is s-bridgeless, $B_{s}(G)=B(G)$ and thus $E(G)=$ $E\left(G_{1}\right) \cup E\left(G_{2}\right)$. It is obvious that $G_{2}-E_{N}(G)$ is acyclic and $\mathcal{F}_{2}$ is a desired signed circuit $\{1,2,3\}$-cover of $G_{2}$.

In particular, if $G$ is g-bridgeless with even negativeness, then $B_{g}(G)=B(G)$ and by Lemma 3.4.1, $H$ has a generalized barbell, denoted by $G_{2}$, containing all edges of $B_{g}(H) \cup\left(\cup_{e \in E_{N}(H)} S_{H}(e)\right)\left(\supseteq B_{g}(G) \cup\left(\cup_{e \in E_{N}(G)} S_{G}(e)\right)\right)$. Thus $E(G)=E\left(G_{1}\right) \cup E\left(G_{2}\right)$, $G_{2}-E_{N}(G)$ is acyclic and by Lemma $3.3 .3, G_{2}$ has a signed circuit double cover. This proves Lemma 3.2.3.

\subsection{Proof of Theorem 3.2.2}

In this section, we complete the proof of Theorems 3.2.2 by applying Lemma 3.2.3. Let $G$ be an s-bridgeless signed graph with $\epsilon_{N}(G)>0$. We only need to consider the case $\left|E_{N}(G)\right|=\epsilon_{N}(G)$ since the existence and the length of a signed circuit cover are two invariants under the switching operations.

Since $G$ is s-bridgeless and $\epsilon_{N}(G)>0$, we have that $\left|E_{N}(G)\right|=\epsilon_{N}(G) \geq 2$. If $G$ contains positive loops, then we may consider the subgraph obtained from $G$ by deleting all positive loops. Thus we further assume that $G$ contains no positive loops. 
By Lemma 3.2.3, $G$ has a bridgeless unsigned subgraph $G_{1}$ and a signed subgraph $G_{2}$ such that $E\left(G_{1}\right) \cup E\left(G_{2}\right)=E(G), G_{2}-E_{N}(G)$ is acyclic and $G_{2}$ has a signed circuit $\{1,2, \ldots, k\}$-cover $\mathcal{F}_{2}$, where $k=2$ if $G$ is g-bridgeless with even negativeness and $k=3$ otherwise.

Note that $E\left(G_{1}\right) \subseteq G-E_{N}(G)$ and thus $E\left(G_{1}\right) \cap E\left(G_{2}\right) \subseteq E\left(G_{2}\right)-E_{N}(G)$ is acyclic. Hence we have the following two inequalities.

$$
\begin{aligned}
\left|E\left(G_{1}\right)\right|+\left|E\left(G_{2}\right)\right| & =\left|E\left(G_{1}\right) \cup E\left(G_{2}\right)\right|+\left|E\left(G_{1}\right) \cap E\left(G_{2}\right)\right| \\
& \leq|E(G)|+|V(G)|-1 . \\
\left|E\left(G_{2}\right)\right| & \leq(|V(G)|-1)+\left|E_{N}(G)\right|=|V(G)|-1+\epsilon_{N}(G) .
\end{aligned}
$$

Let $\mathcal{F}_{2}^{\prime}$ be a subset of $\mathcal{F}_{2}$ such that $\mathcal{F}_{2}^{\prime}$ is still a signed circuit cover of $G_{2}$ and the number of signed circuits of $\mathcal{F}_{2}^{\prime}$ is as small as possible. We have the following claim.

Claim 15. $\ell\left(\mathcal{F}_{2}^{\prime}\right)=\left|E\left(G_{2}\right)\right| \leq k\left|E\left(G_{2}\right)\right|-2(k-1)$.

Proof. Let $t$ be the number of signed circuits in $\mathcal{F}_{2}^{\prime}$. Since $\left|E_{N}\left(G_{2}\right)\right|=\left|E_{N}(G)\right| \geq 2$, $t \geq 1$. By the choice of $\mathcal{F}_{2}^{\prime}$, every signed circuit in $\mathcal{F}_{2}^{\prime}$ has an edge which is covered by $\mathcal{F}_{2}^{\prime}$ exactly once, and so $G_{2}$ has at least $t$ edges which are covered by $\mathcal{F}_{2}^{\prime}$ exactly once. Note that $k=2$ or 3 , and each signed circuit in $\mathcal{F}_{2}$ is of length at least 2 since $G$ has no positive loops. If $t=1$, then $G_{2}$ is the unique signed circuit in $\mathcal{F}_{2}^{\prime}$, and thus $\ell\left(\mathcal{F}_{2}^{\prime}\right)=\left|E\left(G_{2}\right)\right| \leq k\left|E\left(G_{2}\right)\right|-2(k-1)$. If $t \geq 2$, then $\ell\left(\mathcal{F}_{2}^{\prime}\right) \leq k\left(\left|E\left(G_{2}\right)\right|-t\right)+t=$ $k\left|E\left(G_{2}\right)\right|-(k-1) t \leq k\left|E\left(G_{2}\right)\right|-2(k-1)$.

Since $G_{1}$ is bridgeless and unsigned, it follows from Theorem 1.4.3 and 1.4.5 that $G_{1}$ has a circuit cover $\mathcal{F}_{1}$ with total length

$$
\ell\left(\mathcal{F}_{1}\right) \leq \min \left\{\frac{5}{3}\left|E\left(G_{1}\right)\right|,\left|E\left(G_{1}\right)\right|+\left|V\left(G_{1}\right)\right|-1\right\} .
$$

Therefore, $\mathcal{F}=\mathcal{F}_{1} \cup \mathcal{F}_{2}^{\prime}$ is a signed circuit cover of $G$ and by Claim 15 and Equation (3.3) 
together with Equations (3.1) and (3.2), the total length of $\mathcal{F}$ satisfies that

$$
\begin{aligned}
\ell(\mathcal{F})= & \ell\left(\mathcal{F}_{1}\right)+\ell\left(\mathcal{F}_{2}^{\prime}\right) \\
\leq & \min \left\{\frac{5}{3}\left|E\left(G_{1}\right)\right|,\left|E\left(G_{1}\right)\right|+\left|V\left(G_{1}\right)\right|-1\right\}+k\left|E\left(G_{2}\right)\right|-2(k-1) \\
\leq & \min \left\{\frac{5}{3}(|E(G)|+|V(G)|-1)+\left(k-\frac{5}{3}\right)\left(|V(G)|-1+\epsilon_{N}(G)\right)-2(k-1),\right. \\
& \left.(|E(G)|+|V(G)|-1)+(|V(G)|-1)+(k-1)\left(|V(G)|-1+\epsilon_{N}(G)\right)-2(k-1)\right\} \\
= & \min \left\{\frac{5}{3}|E(G)|+k|V(G)|+\left(k-\frac{5}{3}\right) \epsilon_{N}(G)-(3 k-2),\right. \\
\mid & \left.E(G)|+(k+1)| V(G) \mid+(k-1) \epsilon_{N}(G)-(3 k-1)\right\} .
\end{aligned}
$$

This completes the proof of Theorem 3.2.2. 


\section{Chapter 4}

\section{Vector Flows and Integer Flows}

\subsection{Notation and Terminology}

Let $(D, \boldsymbol{\Phi})$ be a vector $S^{1}$-flow of a graph $G$ with flow values

$$
\{\boldsymbol{\Phi}(e): e \in E(G)\}=\left\{ \pm \boldsymbol{\alpha}_{1}, \pm \boldsymbol{\alpha}_{2}, \ldots, \pm \boldsymbol{\alpha}_{b}\right\}
$$

where the set $\left\{\boldsymbol{\alpha}_{1}, \boldsymbol{\alpha}_{2}, \ldots, \boldsymbol{\alpha}_{b}\right\}$ consists of $b$ pairwise linearly independent vectors of $S^{1}$. We may further assume $\{\boldsymbol{\Phi}(e): e \in E(G)\}=\left\{\boldsymbol{\alpha}_{1}, \boldsymbol{\alpha}_{2}, \ldots, \boldsymbol{\alpha}_{k}\right\}$ since otherwise we may reverse the orientations of all edges with flow values $-\boldsymbol{\alpha}_{i}$ and negate their flow values. An edge $e$ is an $\boldsymbol{\alpha}_{i}$-edge of $G$ if $\boldsymbol{f}(e)=\boldsymbol{\alpha}_{i}$. For each $1 \leq i \leq b$, we denote the collection of all $\boldsymbol{\alpha}_{i}$-edges by $E_{i}(G)$.

Definition 4.1.1. Let $(D, \boldsymbol{\Phi})$ be a vector $S^{1}$-flow of a graph $G$ and $\left\{\boldsymbol{\alpha}_{1}, \boldsymbol{\alpha}_{2}, \ldots, \boldsymbol{\alpha}_{b}\right\}$ be the set of flow values. For each vertex $v$ of $G$, define a vector by

$$
\boldsymbol{\epsilon}(v)=<\epsilon_{1}(v), \epsilon_{2}(v), \ldots, \epsilon_{b}(v)>,
$$

where $\epsilon_{i}(v)=\epsilon_{i}^{+}(v)-\epsilon_{i}^{-}(v), \epsilon_{i}^{+}(v)=\left|E^{+}(v) \cap E_{i}(G)\right|$, and $\epsilon_{i}^{-}(v)=\left|E^{-}(v) \cap E_{i}(G)\right|$ for each $1 \leq i \leq b$. The vector $\boldsymbol{\epsilon}(v)$ is the balanced vector at vertex $v$. A vertex $v$ is trivial if $\boldsymbol{\epsilon}(v)=\mathbf{0}$, and is unsplittable if either $\epsilon_{i}^{+}(v)$ or $\epsilon_{i}^{-}(v)$ is zero for each $1 \leq i \leq b$. The 
balanced equation of a vertex $v$ is defined as

$$
\epsilon_{1}(v) \boldsymbol{\alpha}_{1}+\epsilon_{2}(v) \boldsymbol{\alpha}_{2}+\cdots+\epsilon_{b}(v) \boldsymbol{\alpha}_{b}=\mathbf{0}
$$

Let $S(\boldsymbol{\Phi})$ be the linear subspace of $\mathbb{R}^{b}$ generated by all balanced vectors of $(D, \boldsymbol{\Phi})$ of $G$. The rank of the subspace $S(\boldsymbol{\Phi})$, denoted $\operatorname{rank}(\boldsymbol{\Phi})$, is also defined as the rank of the vector $S^{1}$-flow $(D, \boldsymbol{\Phi})$.

\subsection{Vector $S^{1}$-flows of the dual of unit distance graph}

In [45], Thomassen characterized planar graphs admitting vector $S^{1}$-flows as follows.

Lemma 4.2.1 (Thomassen [45]). Let $G$ be a planar graph. Then $G$ admits a vector $S^{1}$ flow if and only if the dual graph $G^{*}$ is homomorphic to a subgraph of a unit distance graph, where a unit distance graph is a graph whose vertices are points in Euclidian plane $\mathbb{R}^{2}$ such that two vertices are adjacent if and only if they have distance 1.

Based on Lemma 4.2.1, Thomassen [45] constructed a family of graphs that admit vector $S^{1}$-flows but no integer 3 -NZFs. One of them, denoted by $G$, is illustrated in Figure 4.1-(a). The graph $G$ admits a vector $S^{1}$-flow since its dual graph is homomorphic to a unit distance graph $G^{*}$ (see Figure 4.1-(b)). With no confusion and slight abuse of notation, let $G^{*}$ be the dual of $G$. Note that $G^{*}$ is the Hajós graph [4] with chromatic number 4. By Tutte's Theorem [49], $G$ admits an integer 4-NZF but no integer 3-NZF. In fact, $G^{*}$ can be obtained from two $K_{4}$ by Hajós construction. Repeatedly adding $K_{4}$ to $G^{*}$ by Hajós construction, we can construct an infinite family of counterexamples.

Since $G^{*}$ is a unit distance graph, each edge of $G^{*}$ can be considered as a unit vector under some orientation $D^{*}$. Observe that there are seven pairwise linearly independent unit vectors among 11 edges of $G^{*}$. If we associate the vectors and orientations of edges in $G^{*}$ to its dual edges in $G$, respectively, then we obtain a vector $S^{1}$-flow of $G$ with orientation $D$. From Figure 4.1-(b), one can easily see that the following is the set of all balanced vectors of the vector $S^{1}$-flow

$$
\{ \pm<1,1,1,0,0,0,0>, \pm<0,0,0,0,1,1,1>, \pm<1,1,0,1,0,1,1>\}
$$




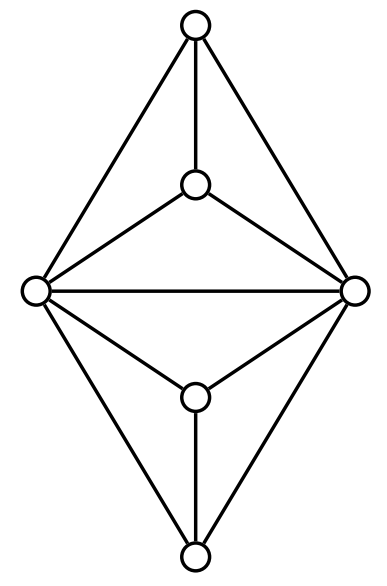

(a): graph $G$

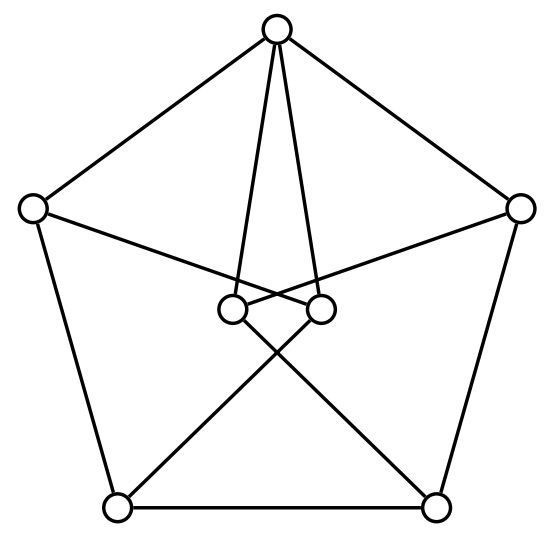

(b): graph $G^{*}$

Figure 4.1: Thomassen's counterexample $G$

Thus, the rank of the vector flow is three since they are linearly independent.

Inspired by Theorem 1.5.7 and the above discussion related to the counterexample, we propose the following problem.

Problem 4.2.2. Find an integer $r$ such that if a graph $G$ admits a vector $S^{1}$-flow $(D, \Phi)$ with rank at most $r$, then $G$ admits an integer $4-N Z F$.

Jain [22] also proposed another vector flow conjecture related to Tutte's 5-flow conjecture. It says that every bridgeless graph admits a vector $S^{2}$-flow. Inspired by this, we proposed another problem as follows.

Problem 4.2.3. Find an integer $r$ such that if a graph $G$ admits a vector $S^{2}$-flow $(D, \Phi)$ with rank at most $r$, then $G$ admits an integer 5-NZF. 


\subsection{Proof of Theorem 1.5.7}

\subsubsection{Vector $S^{1}$-flows with rank one}

The following lemma is a direct consequence of Definition 2.2.1.

Lemma 4.3.1. Let $(D, \Phi)$ be a vector $S^{1}$-flow of a graph $G$. Let $v \in V(G)$ and $A \subseteq E(v)$ such that

$$
\sum_{e \in A \cap E_{D}^{+}(v)} \boldsymbol{\Phi}(e)=\sum_{e \in A \cap E_{D}^{-}(v)} \boldsymbol{\Phi}(e) .
$$

Then

(1) The graph $G_{(v ; A)}$ admits a vector $S^{1}$-flow $\left(D_{A}, \mathbf{\Phi}_{A}\right)$ and $S\left(\mathbf{\Phi}_{A}\right)=S(\mathbf{\Phi})$.

(2) If $G_{(v ; A)}$ has a modulo k-orientation, then so does $G$.

Definition 4.3.2 (Elementary Balanced Vector). Let $(D, \Phi)$ be a vector $S^{1}$-flow of a graph $G$ with set of flow values $\left\{\boldsymbol{\alpha}_{1}, \boldsymbol{\alpha}_{2}, \ldots, \boldsymbol{\alpha}_{b}\right\}$ and $\operatorname{rank}(\boldsymbol{\Phi}) \leq 1$. Let $\boldsymbol{\epsilon}=<$ $\epsilon_{1}, \epsilon_{2}, \ldots, \epsilon_{b}>$ be a vector such that either $\boldsymbol{\epsilon}=\mathbf{0}$ when $S(\boldsymbol{\Phi})=\{\mathbf{0}\}$, or $\boldsymbol{\epsilon}$ is a nonzero vector in $S(\boldsymbol{\Phi})$ satisfying that

(1) all coordinates of $\boldsymbol{\epsilon}$ are integers;

(2) subject to (1), $\|\boldsymbol{\epsilon}\|=\sum_{i=1}^{b}\left|\epsilon_{i}\right|$ is as small as possible.

Then the vector $\boldsymbol{\epsilon}$ is the elementary balanced vector of $(D, \boldsymbol{\Phi})$. Clearly, $\boldsymbol{\epsilon}$ is unique up to scalar multiplication of -1 . Its length $k^{*}$ is defined by

$$
k^{*}=\|\boldsymbol{\epsilon}\|=\sum_{i=1}^{b}\left|\epsilon_{i}\right| .
$$

In the following context, if $(D, \boldsymbol{\Phi})$ is a vector $S^{1}$-flow of a graph $G$, then we denote by $\left\{\boldsymbol{\alpha}_{1}, \boldsymbol{\alpha}_{2}, \ldots, \boldsymbol{\alpha}_{b}\right\}$ the set of flow values. If $\operatorname{rank}(\boldsymbol{\Phi})=1$, we further denote by $\boldsymbol{\epsilon}=<$ $\epsilon_{1}, \epsilon_{2}, \ldots, \epsilon_{b}>$ the elementary balanced vector and $k^{*}$ the length of $\boldsymbol{\epsilon}$. 
Lemma 4.3.3. Let $(D, \Phi)$ be a vector $S^{1}$-flow of a graph $G$ with $\operatorname{rank}(\boldsymbol{\Phi}) \leq 1$. If $G$ has at least one nontrivial vertex, then $k^{*} \geq 3$ and for each vertex $v$, either $v$ is unsplittable and $\operatorname{deg}(v)$ is a multiple of $k^{*}$, or $v$ is splittable. Furthermore, if $v$ is unsplittable, then $v$ can be split into $\frac{\operatorname{deg}(v)}{k^{*}}$ vertices such that the balanced vector of each new vertex is either $\boldsymbol{\epsilon}$ or $-\boldsymbol{\epsilon}$.

Proof. (1) Since $G$ has at least one nontrivial vertex, $\boldsymbol{\epsilon} \neq \mathbf{0}$. Note that $k^{*} \neq 1,2$ by Equation (4.1) and Definition 4.3.2. This implies $k^{*} \geq 3$.

(2) It suffices to prove that each unsplittable vertex $v$ has the balanced vector $\boldsymbol{\epsilon}(v)=s \boldsymbol{\epsilon}$ for some integer $s$. Since $(D, \boldsymbol{\Phi})$ has rank one, for each vertex $v$ in $G$, there exists some $s \in \mathbb{Q}$ such that $\boldsymbol{\epsilon}(v)=s \boldsymbol{\epsilon}$. Suppose to the contrary $s=\frac{p}{q}$, where $p, q$ are integers, $|q|>1$ and $\operatorname{gcd}(p, q)=1$. Then there exist two integers $a, b$ such that $p=a q+b$ and $0<|b|<|q|$. Thus, $\frac{b}{q} \boldsymbol{\epsilon}=\boldsymbol{\epsilon}(v)-a \boldsymbol{\epsilon}$ is an integer vector but has smaller length than $\boldsymbol{\epsilon}$, which contradicts the definition of $\boldsymbol{\epsilon}$.

Let $G$ be a graph admitting a vector $S^{1}$-flow $(D, \boldsymbol{\Phi})$ with rank one. If $G$ contains some splittable nontrivial vertex $v$ (that is, there exists at least one pair of edges incident with $v$ with the same flow value but opposite orientations), then one can repeatedly split all possible pairs of edges at $v$ and other splittable vertices until no more splittable vertices. Let $G^{\prime}$ be the resulting graph. By Lemma 4.3.3-(2), for each vertex $v$ of $G^{\prime}$, one can split $v$ into $\frac{\operatorname{deg}_{G^{\prime}}(v)}{k^{*}}$ vertices such that each new vertex has degree $k^{*}$ and has the balanced vector either $\boldsymbol{\epsilon}$ or $-\boldsymbol{\epsilon}$. The resulting graph is defined as the core of $G$.

The following is a straightforward observation of Lemma 4.3.1 and definition of core.

Proposition 4.3.4. Let $G$ be a graph admitting a vector $S^{1}$-flow $(D, \Phi)$ with rank one. If $H$ is the core of $G$, then

(1) it is a $k^{*}$-regular bipartite graph with vertex bipartition $(A, B)$, where

$$
A=\{v \in V(H): \boldsymbol{\epsilon}(v)=\boldsymbol{\epsilon}\} \quad \text { and } \quad B=\{v \in V(H): \boldsymbol{\epsilon}(v)=-\boldsymbol{\epsilon}\} .
$$

(2) it admits a vector $S^{1}$-flow, say $\left(D_{h}, \mathbf{\Phi}_{h}\right)$, and $S\left(\mathbf{\Phi}_{h}\right)=S(\mathbf{\Phi})$. 
(3) it has a modulo $k^{*}$-orientation and therefore $G$ has a modulo $k^{*}$-orientation.

(4) for each $1 \leq i \leq b, E_{i}(H)$ has a decomposition into $\left|\epsilon_{i}\right|$ perfect matchings.

Remark. Orienting all edges of $H$ from $A$ to $B$, the resulting orientation is a modulo $k^{*}$-orientation of $H$. Meanwhile, $E_{i}(H)$ has a perfect matching decomposition since the subgraph induced by $E_{i}(G)$ is an $\left|\epsilon_{i}\right|$-regular bipartite spanning subgraph of $H$.

Theorem 4.3.5. If a graph $G$ admits a vector $S^{1}$-flow $(D, \boldsymbol{\Phi})$ with $\operatorname{rank}(\boldsymbol{\Phi}) \leq 1$, then either $G$ is eulerian and thus admits an integer $2-N Z F$, or $G$ admits an integer circular $\left(2+\frac{1}{p}\right)$-flow for some integer $p$ where $k^{*}=2 p+1$ and $p \geq 1$.

Proof. If $k^{*}=0$, then $v$ is trivial and $\operatorname{deg}_{G}(v)$ is even for each vertex $v$ of $G$. This implies that $G$ is eulerian and thus admits an integer $2-\mathrm{NZF}$.

Now we assume $k^{*}>0$. Then $G$ contains at least one nontrivial vertex and thus $G$ admits a modulo $k^{*}$-orientation by Proposition 4.3.4-(3). If $k^{*}$ is even, then $G$ is eulerian and admits an integer 2-NZF. Otherwise, by Theorem 1.3.11, $G$ admits an integer circular $\left(2+\frac{1}{p}\right)$-flow with $k^{*}=2 p+1$.

\subsubsection{Some useful lemmas}

In this subsection, we present some lemmas which will be applied in the proof of the case of vector $S^{1}$-flows with rank two.

Lemma 4.3.6. Let $\left\{\boldsymbol{\alpha}_{1}, \boldsymbol{\alpha}_{2}, \ldots, \boldsymbol{\alpha}_{b}\right\}$ be a set of $b$ pairwise linearly independent vectors in $S^{1}$. Then the following statements hold.

(1) If $\sum_{i=1}^{b} \epsilon_{i} \boldsymbol{\alpha}_{i}=\mathbf{0}$ for some nonzero integers $\epsilon_{1}, \epsilon_{2}, \ldots, \epsilon_{b}$, then

(1-a) for each $j$ with $1 \leq j \leq b$,

$$
\left|\epsilon_{j}\right|<\sum_{i \neq j}\left|\epsilon_{i}\right|
$$


(1-b) $\sum_{i=1}^{b}\left|\epsilon_{i}\right| \neq 1,2,4$.

(2) If $\boldsymbol{\alpha}_{1}+$ t $\boldsymbol{\alpha}_{2}+t \boldsymbol{\alpha}_{3}=\mathbf{0}$ for some integer $t$, then for any nonzero integers $\epsilon_{2}, \epsilon_{3}, \epsilon_{4}$ with $\left|\epsilon_{4}\right|=1$, we have

$$
\epsilon_{2} \boldsymbol{\alpha}_{2}+\epsilon_{3} \boldsymbol{\alpha}_{3}+\epsilon_{4} \boldsymbol{\alpha}_{4} \neq \mathbf{0}
$$

Proof. (1-a) Note that $b \geq 3$ since each $\epsilon_{i}$ is nonzero. For each $j$ with $1 \leq j \leq b$, we have $\epsilon_{j} \boldsymbol{\alpha}_{j}=-\sum_{i \neq j} \epsilon_{i} \boldsymbol{\alpha}_{i}$. Thus,

$$
\left|\epsilon_{j}\right|=\left|\epsilon_{j} \boldsymbol{\alpha}_{j}\right|=\left|\sum_{i \neq j} \epsilon_{i} \boldsymbol{\alpha}_{i}\right| \leq\left|\sum_{i \neq j}\right| \epsilon_{i} \mid
$$

with equality holds only if all $\boldsymbol{\alpha}_{i}$ 's are pairwise linearly dependent. Thus, $\left|\epsilon_{j}\right|<\sum_{i \neq j}\left|\epsilon_{i}\right|$.

(1-b) Clearly, $\sum_{i=1}^{b}\left|\epsilon_{i}\right| \neq 1,2$. Suppose to the contrary $\sum_{i=1}^{b}\left|\epsilon_{i}\right|=4$. By (1-a), $2\left|\epsilon_{j}\right|<\sum_{i=1}^{b}\left|\epsilon_{i}\right|=4$ and therefore $\left|\epsilon_{j}\right| \leq 1$ for each $j=1,2, \ldots, b$. Without lose of generality, we assume $\left|\epsilon_{i}\right|=1$ for each $i=1,2,3,4$ and $\epsilon_{j}=0$ for each $j \geq 5$. Then

$$
\epsilon_{1} \boldsymbol{\alpha}_{1}+\epsilon_{2} \boldsymbol{\alpha}_{2}+\epsilon_{3} \boldsymbol{\alpha}_{3}+\epsilon_{4} \boldsymbol{\alpha}_{4}=\mathbf{0}
$$

This implies that the vectors $\epsilon_{1} \boldsymbol{\alpha}_{1}, \epsilon_{2} \boldsymbol{\alpha}_{2}, \epsilon_{3} \boldsymbol{\alpha}_{3}$ and $\epsilon_{4} \boldsymbol{\alpha}_{4}$ form a parallelogram in $\mathbb{R}^{2}$, which contradicts the assumption that they are pairwise linearly independent vectors.

(2) Suppose $\epsilon_{2} \boldsymbol{\alpha}_{2}+\epsilon_{3} \boldsymbol{\alpha}_{3}+\epsilon_{4} \boldsymbol{\alpha}_{4}=\mathbf{0}$. Since $\epsilon_{4}= \pm 1$, we have $\left|\epsilon_{2}\right|=\left|\epsilon_{3}\right|$ by (1-a). If $\epsilon_{2}=-\epsilon_{3}$, then by equations $\boldsymbol{\alpha}_{1}+t \boldsymbol{\alpha}_{2}+t \boldsymbol{\alpha}_{3}=\mathbf{0}$ and $\epsilon_{2} \boldsymbol{\alpha}_{2}-\epsilon_{2} \boldsymbol{\alpha}_{3}+\epsilon_{4} \boldsymbol{\alpha}_{4}=\mathbf{0}$, we have

$$
\epsilon_{2} \boldsymbol{\alpha}_{1}+2 t \epsilon_{2} \boldsymbol{\alpha}_{2}+t \epsilon_{4} \boldsymbol{\alpha}_{4}=\mathbf{0} .
$$

Since $\left|\epsilon_{2}\right| \geq 1,|t| \geq 1$, and $\left|\epsilon_{4}\right|=1$, we have

$$
\left|2 t \epsilon_{2}\right|=\left|t \epsilon_{2}\right|+\left|t \epsilon_{2}\right| \geq\left|\epsilon_{2}\right|+\left|t \epsilon_{4}\right|,
$$

which contradicts (1-a).

If $\epsilon_{2}=\epsilon_{3}$, then by equations $\boldsymbol{\alpha}_{1}+t \boldsymbol{\alpha}_{2}+t \boldsymbol{\alpha}_{3}=\mathbf{0}$ and $\epsilon_{2} \boldsymbol{\alpha}_{2}+\epsilon_{2} \boldsymbol{\alpha}_{3}+\epsilon_{4} \boldsymbol{\alpha}_{4}=\mathbf{0}$, we have

$$
\epsilon_{2} \boldsymbol{\alpha}_{1}+t \epsilon_{4} \boldsymbol{\alpha}_{4}=\mathbf{0}
$$

implying that $\boldsymbol{\alpha}_{1}$ and $\boldsymbol{\alpha}_{4}$ are linearly dependent, a contradiction. 
Lemma 4.3.7. Let $(D, \Phi)$ be a vector $S^{1}$-flow of a graph $G$ with $\operatorname{rank}(\boldsymbol{\Phi}) \leq 1$. If $k^{*} \leq 5$, then $G$ has a modulo 3-orientation $D^{\prime}$ such that either $D^{\prime}=D$ or $D^{\prime}$ can be obtained from $D$ by reversing the orientations of all edges of some $E_{i}(G)$ 's.

Proof. If $k^{*}=0$, let $D^{\prime}=D$ and $D$ itself is a modulo 3-orientation of $G$. Otherwise, $G$ contains at least one nontrivial vertex and $k^{*} \in\{3,5\}$ by Lemma 4.3.6-(1-b). Let $H$ be the core of $G$. By Proposition 4.3.4-(2), $H$ admits a vector $S^{1}$-flow, say $\left(D_{h}, \boldsymbol{\Phi}_{h}\right)$. Based on the definition of the core, it suffices to show that $H$ has a modulo 3-orientation $D_{h}^{\prime}$ which can be obtained from $D_{h}$ by reversing the orientations of all edges of some $E_{i}(H)$ 's.

By Proposition 4.3.4-(1),

$$
\left|\operatorname{deg}_{D_{h}}^{+}(v)-\operatorname{deg}_{D_{h}}^{-}(v)\right|=\left|\sum_{i=1}^{b} \epsilon_{i}(v)\right|=\left|\sum_{i=1}^{b} \epsilon_{i}\right| .
$$

Thus, if $\sum_{i=1}^{b} \epsilon_{i} \equiv 0(\bmod 3)$, then let $D_{h}^{\prime}=D_{h}$ and $D_{h}$ itself is a modulo 3-orientation of $H$. Next we assume $\sum_{i=1}^{b} \epsilon_{i} \not \equiv 0(\bmod 3)$.

If $k^{*}=3$, by Lemma 4.3.6-(1-a), $\boldsymbol{\epsilon}$ has exactly three coordinates with absolute value equal to 1 . Without loss of generality, we assume $\left|\epsilon_{i}\right|=1$ for each $i=1,2,3$ and $\epsilon_{j}=0$ for all $j$ with $4 \leq j \leq b$. By Proposition 4.3.4-(4), $E_{i}(H)$ is a perfect matching of $H$ for each $i=1,2,3$. Thus, we can obtain a modulo 3 -orientation from $D_{h}$ by reversing the orientation of all edges of $E_{i}(H)$ for each $i$ with $\epsilon_{i}=-1$.

Now we assume $k^{*}=5$. Then $\left|\epsilon_{i}\right| \leq 2$ for each $i$ by Lemma 4.3.6-(1-a). Note that $E_{i}(H)$ is a perfect matching if $\left|\epsilon_{i}\right|=1$. We first assume $\left|\epsilon_{i}\right|=\left|\epsilon_{j}\right|=1$ for some $i \neq j$. If $\epsilon_{i}=\epsilon_{j}$, then we reverse the orientations of all edges of $E_{i}(H)$ and all edges of $E_{t}(H)$ for each $t \neq i, j$ and $\epsilon_{t}<0$. If $\epsilon_{i}=-\epsilon_{j}$, we reverse the orientations of all edges of $E_{t}(H)$ for each $t \neq i, j$ and $\epsilon_{t}<0$. In either case, we obtain a modulo 3-orientation $D_{h}^{\prime}$ of $H$.

Now we assume that $\boldsymbol{\epsilon}$ has at most one coordinate with absolute value 1 . Without loss of generality, we assume either $\boldsymbol{\epsilon}=(1,-2,2)$ or $\boldsymbol{\epsilon}=(1,2,2)$. By Proposition 4.3.4$(4), E_{2}(H)$ and $E_{3}(H)$ induce two edge-disjoint spanning 2-regular subgraphs of $H$, and $E_{1}(H)$ is a perfect matching. If $\boldsymbol{\epsilon}=(1,-2,2)$, we reverse the orientations of all edges of $E_{3}(H)$. If $\boldsymbol{\epsilon}=(1,2,2)$, we reverse the orientations of all edges of $E_{1}(H)$. In either case, 
one can obtain a modulo 3 -orientation $D_{h}^{\prime}$ of $H$.

Lemma 4.3.8. Let $(D, \Phi)$ be a vector $S^{1}$-flow of a graph $G$ with rank one. If the vector $\boldsymbol{\epsilon}=<\epsilon_{1}, \epsilon_{2}, \ldots, \epsilon_{a}, \ldots, \epsilon_{b}>$ satisfies $\left|\sum_{j>a} \epsilon_{i}\right| \geq 2$ for some $1 \leq a<b$, then $G$ has $a$ modulo 3-orientation $D^{\prime}$ such that $D^{\prime}$ agrees with $D$ on $\bigcup_{i=1}^{a} E_{i}(G)$.

Proof. Similar to the discussion in Lemma 4.3.7, it suffices to prove that the core of $G$, say $H$, has a modulo 3-orientation $D_{h}^{\prime}$ such that $D_{h}^{\prime}$ agrees with $D_{h}$ on $\bigcup_{i=1}^{a} E_{i}(H)$, where $H$ is the core of $G$. We further assume $\sum_{i=1}^{b} \epsilon_{i} \equiv t(\bmod 3)$ for some $t=1$ or 2 .

By Proposition 4.3.4-(1) and $\left|\sum_{j>a} \epsilon_{i}\right| \geq 2, H$ has a vertex bipartition $(A, B)$ and has two edge-disjoint perfect matchings, say $M_{1}$ and $M_{2}$, such that $M_{1} \subseteq E_{j_{1}}(H), M_{2} \subseteq$ $E_{j_{2}}(H)$ for some $j_{1}, j_{2}>a$ (it is possible that $j_{1}=j_{2}$ ), and, $M_{1}, M_{2}$ have the same orientation either both from $A$ to $B$ or both from $B$ to $A$. One can obtain a modulo 3orientation by reversing the orientations of all edges of $2 t(\bmod 3) \operatorname{members}$ of $\left\{M_{1}, M_{2}\right\}$ in $D_{h}$.

Lemma 4.3.9. Let $(D, \boldsymbol{\Phi})$ be a vector $S^{1}$-flow of a graph $G$ with rank two. Let $\{\boldsymbol{\eta}, \boldsymbol{\phi}\}$ be two integer vectors such that

(1) $\{\boldsymbol{\eta}, \boldsymbol{\phi}\}$ is a basis of $S(\boldsymbol{\Phi})$;

(2) $\boldsymbol{\eta}=<\eta_{1}=1, \eta_{2}, \ldots, \eta_{a}, 0, \ldots, 0>$ and $\boldsymbol{\phi}=<\phi_{1}=0, \phi_{2}, \ldots \ldots, \phi_{b}>$;

(3) the greatest common divisor of nonzero coordinates in $\phi$ is 1.

If $V(G)$ can be partitioned into $V_{1}$ and $V_{2}$, where

$$
V_{1}=\{v \in V(G): \boldsymbol{\epsilon}(v)= \pm \boldsymbol{\eta}\} \quad \text { and } \quad V_{2}=\{v \in V(G): \boldsymbol{\epsilon}(v)= \pm \boldsymbol{\phi}\}
$$

then $G$ has a modulo 3-orientation if one of the following statements is satisfied:

(1) $\sum_{i=1}^{a}\left|\eta_{i}\right| \leq 5$ and $\left|\sum_{j>a}^{b} \phi_{j}\right| \geq 2$.

(2) $\sum_{i=1}^{a} \eta_{i} \equiv 2(\bmod 3)$, and for some $2 \leq j \leq a, \eta_{j}=1$ and $\left|\phi_{j}\right| \geq 2$. 
Proof. We can assume that each vertex of $G$ is unsplittable by Lemma 4.3.1-(2). Let

$$
\begin{array}{cc}
V_{11}=\{v \in V(G): \boldsymbol{\epsilon}(v)=\boldsymbol{\eta}\}, & V_{12}=\{v \in V(G): \boldsymbol{\epsilon}(v)=-\boldsymbol{\eta}\}, \\
V_{21}=\{v \in V(G): \boldsymbol{\epsilon}(v)=\boldsymbol{\phi}\}, & V_{22}=\{v \in V(G): \boldsymbol{\epsilon}(v)=-\boldsymbol{\phi}\} .
\end{array}
$$

Thus, $G\left[V_{j}\right]$ is a bipartite graph with vertex bipartition $\left(V_{j 1}, V_{j 2}\right)$ for each $j=1,2$. Note that $E_{1}(G)$ is a perfect matching of $G\left[V_{1}\right]$. Thus, $\left|V_{11}\right|=\left|V_{12}\right|=\frac{1}{2}\left|V_{1}\right|$ and the balanced vector of the edge-cut $\left[V_{1}, V_{2}\right]$ is

$$
\sum_{v \in V_{1}} \boldsymbol{\epsilon}(v)=\sum_{v \in V_{11}} \boldsymbol{\eta}+\sum_{v \in V_{12}}-\boldsymbol{\eta}=\mathbf{0}
$$

Case I. $\sum_{i=1}^{a}\left|\eta_{i}\right| \leq 5$ and $\left|\sum_{j>a}^{b} \phi_{j}\right| \geq 2$.

Let $G_{i}$ be the graph obtained from $G$ by contracting $V_{3-i}$ into a new vertex $v_{3-i}$ for each $i=1,2$. Let $\left(D_{1}, \boldsymbol{\Phi}_{1}\right)$ be the restriction of the vector $S^{1}$-flow $(D, \boldsymbol{\Phi})$ of $G$ to $G_{1}$. Then $\left(D_{1}, \boldsymbol{\Phi}_{1}\right)$ is a vector $S^{1}$-flow of $G_{1}$. By Equation $(4.2), \boldsymbol{\epsilon}\left(v_{2}\right)=\mathbf{0}$ and thus $\left(D_{1}, \boldsymbol{\Phi}_{1}\right)$ is a vector $S^{1}$-flow with rank one. Since $\eta_{1}=1$, the vector $\boldsymbol{\eta}$ is the elementary balanced vector of $\left(D_{1}, \boldsymbol{\Phi}_{1}\right)$.

Since $\sum_{i=1}^{a}\left|\eta_{i}\right| \leq 5$, by Lemma 4.3.7, $G_{1}$ has a modulo 3 -orientation $D_{1}^{\prime}$ such that either $D_{1}^{\prime}=D_{1}$ or $D_{1}^{\prime}$ can be obtained from $D_{1}$ by reversing the orientations of all edges of some $E_{i_{j}}\left(G_{1}\right)$ 's. Denote by $E_{i_{j}}\left(G_{1}\right)$ with $j=1, \ldots, t$ the set of all edges whose orientations have been reversed. Note that $i_{j} \leq a$.

Let $D^{\prime}$ be the orientation obtained from $D$ by reversing the orientations of all edges of $\bigcup_{j=1}^{t} E_{i_{j}}(G)$. Thus, $\left(D^{\prime}, \boldsymbol{\Phi}^{\prime}\right)$ is a vector $S^{1}$-flow of $G$ where $\boldsymbol{\Phi}^{\prime}(e)=-\boldsymbol{\Phi}(e)$ if $e \in$ $\bigcup_{j=1}^{t} E_{i_{j}}(G)$ and $\boldsymbol{\Phi}^{\prime}(e)=\boldsymbol{\Phi}(e)$ otherwise. For each $i \leq b$, let $\phi_{i}^{\prime}=-\phi_{i}$ if the orientations of all edges of $E_{i}(G)$ are reversed and $\phi_{i}^{\prime}=\phi_{i}$ otherwise. Since $i_{j} \leq a$ for each $j=1, \ldots, t$, the balanced vector of each vertex $v \in V_{2}$ is either $\phi^{\prime}$ or $-\phi^{\prime}$, where

$$
\phi^{\prime}=<0, \phi_{2}^{\prime}, \ldots, \phi_{a}^{\prime}, \phi_{a+1}, \ldots, \phi_{b}>
$$

Similarly, $G_{2}$ admits a vector $S^{1}$-flow $\left(D_{2}, \boldsymbol{\Phi}_{2}\right)$ which is a restriction of $\left(D^{\prime}, \boldsymbol{\Phi}^{\prime}\right)$ with rank one. Since the greatest common divisor of nonzero coordinates of $\phi$ is 1 , the new 
vector $\phi^{\prime}$ is the elementary balanced vector of $\left(D_{2}, \Phi_{2}\right)$. Since $\left|\sum_{j>a}^{b} \phi_{j}^{\prime}\right|=\left|\sum_{j>a}^{b} \phi_{j}\right| \geq 2$, by Lemma 4.3.8, $G_{2}$ has a modulo 3-orientation $D_{2}^{\prime}$ such that $D_{2}^{\prime}$ agrees with $D_{2}$ on $\bigcup_{i=1}^{a} E_{i}\left(G_{2}\right)$. Since $D_{1}^{\prime}$ and $D_{2}$ are restrictions of $D^{\prime}, D_{1}^{\prime}$ agrees with $D_{2}$ on $\left[V_{1}, V_{2}\right] \subseteq$ $\bigcup_{i=1}^{a} E_{i}\left(G_{2}\right)$. Thus, $D_{1}^{\prime}$ agrees with $D_{2}^{\prime}$ on $\left[V_{1}, V_{2}\right]$. Note that $D_{1}^{\prime} \cap D_{2}^{\prime}=\left[V_{1}, V_{2}\right]$. Therefore, the union orientation $D_{1}^{\prime} \cup D_{2}^{\prime}$ is a proper modulo 3-orientation of the graph $G$.

Case II. $\sum_{i=1}^{a} \eta_{i} \equiv 2(\bmod 3)$, and for some $j$ with $2 \leq j \leq a, \eta_{j}=1$ and $\left|\phi_{j}\right| \geq 2$.

Since each vertex of $G$ is balanced with balanced vector either $\pm \boldsymbol{\eta}$ or $\pm \boldsymbol{\phi}$, for each vertex $v \in V_{1}$ and each vertex $u \in V_{2}$, we have

$$
\left|\operatorname{deg}_{D}^{+}(v)-\operatorname{deg}_{D}^{-}(v)\right|=\left|\sum_{i=1}^{a} \eta_{i}\right| \quad \text { and } \quad\left|\operatorname{deg}_{D}^{+}(u)-\operatorname{deg}_{D}^{-}(u)\right|=\left|\sum_{i=1}^{b} \phi_{i}\right| .
$$

Since $\eta_{1}=1$ and $\phi_{1}=0$, we know that $E_{1}(G)$ is a perfect matching in $G\left[V_{1}\right]$ and no vertex in $V_{2}$ is incident with an $\boldsymbol{\alpha}_{1}$-edge. Let $D_{1}$ be the orientation obtained from $D$ by reversing the orientations of all $\boldsymbol{\alpha}_{1}$-edges of $E_{1}(G)$. Since $\sum_{i=1}^{a} \eta_{i} \equiv 2(\bmod 3)$, for each vertex $v \in V_{1}$ and each vertex $u \in V_{2}$, we have $\left|\operatorname{deg}_{D_{1}}^{+}(v)-\operatorname{deg}_{D_{1}}^{-}(v)\right| \equiv 0(\bmod 3)$, $\operatorname{deg}_{D_{1}}^{+}(u)=\operatorname{deg}_{D}^{+}(u)$, and $\operatorname{deg}_{D_{1}}^{-}(u)=\operatorname{deg}_{D}^{-}(u)$.

If $\sum_{i=2}^{b} \phi_{i} \equiv 0(\bmod 3)$, then $D_{1}$ is a modulo 3-orientation of $G$, done. Now we assume $\sum_{i=2}^{b} \phi_{i} \not \equiv 0(\bmod 3)$ and regard $D_{1}$ as digraphs. Let $L$ be the subgraph of $D_{1}$ induced by $\left(E_{1}(G) \cup E_{j}(G)\right) \cap\left(E\left(G\left[V_{1}\right]\right) \cup E\left(V_{1}, V_{2}\right)\right)$, the set of all $\boldsymbol{\alpha}_{1}$-edges and all $\boldsymbol{\alpha}_{j}$ edges with at least one endpoint in $V_{1}$. Since $\eta_{1}=\eta_{j}=1$, for each vertex $v \in V_{1}$ we have $\operatorname{deg}_{L}^{+}(v)=\operatorname{deg}_{L}^{-}(v)=1$ and for each vertex $u$ in $V_{2} \cap V(L)$ we have either $\operatorname{deg}_{L}^{+}(u)=0$ or $\operatorname{deg}_{L}^{-}(u)=0$. Therefore, $L$ can be decomposed into a collection of edge-disjoint directed circuits with all vertices in $V_{1}$ and directed paths with both endvertices in $V_{2}$. Note that all directed circuits are contained in $G\left[V_{1}\right]$. Let $\left\{P_{1}, \ldots, P_{t}\right\}$ be the collection of directed paths in $L$ and denote by $x_{i}$ and $y_{i}$ the head and tail of $P_{i}$, respectively, for each $1 \leq i \leq t$.

Construct a new digraph $D_{2}$ from $D_{1}$ by replacing each directed path $P_{i}$ with a new arc $e_{i}$ oriented from $y_{i}$ to $x_{i}$ and assigned $\boldsymbol{\alpha}_{j}$ for each $i=1,2, \ldots, t$. Let $K$ be the underlying graph of $D_{2}$ and $\boldsymbol{\Phi}_{2}$ be the resulting "flow" function of $K$. Note that $\left(D_{2}, \boldsymbol{\Phi}_{2}\right)$ is not a vector $S^{1}$-flow of $K$ since all the vertices of $V_{1}$ are not balanced. But each vertex of $V_{2}$ is balanced with balanced vector either $\phi$ or $-\phi$ in $K$. By the construction of 
$K$, each $\boldsymbol{\alpha}_{j}$-edge of $K$ is either in $K\left[V_{2}\right]$ or in the alternating $\left(\boldsymbol{\alpha}_{1}, \boldsymbol{\alpha}_{j}\right)$-circuits of $K\left[V_{1}\right]$. Thus, the subgraph induced by $E_{j}\left(K\left[V_{2}\right]\right)$ is a $\left|\phi_{j}\right|$-regular bipartite graph with bipartition $\left(V_{21}, V_{22}\right)$. Since $\left|\phi_{j}\right| \geq 2$, the graph $K$ has at least two edge-disjoint perfect matchings, say $M_{1}$ and $M_{2}$, such that $M_{1}$ and $M_{2}$ have the same orientation which is either both from $V_{21}$ to $V_{22}$, or both from $V_{22}$ to $V_{21}$.

By the definition of $D_{2}$,

$$
\left|\operatorname{deg}_{D_{2}}^{+}(v)-\operatorname{deg}_{D_{2}}^{-}(v)\right|=\left|\operatorname{deg}_{D_{1}}^{+}(v)-\operatorname{deg}_{D_{1}}^{-}(v)\right| \equiv 0 \quad(\bmod 3)
$$

for each $v \in V_{1}$, and

$$
\left|\operatorname{deg}_{D_{h}}^{+}(2)-\operatorname{deg}_{D_{2}}^{-}(u)\right|=\left|\operatorname{deg}_{D_{1}}^{+}(u)-\operatorname{deg}_{D_{1}}^{-}(u)\right| \equiv r \quad(\bmod 3)
$$

for each $u \in V_{2}$, where $r=1$ or 2 . One can obtain a modulo 3-orientation $D_{2}^{\prime}$ of $K$ by reversing the orientations of $2 r(\bmod 3)$ members of $\left\{M_{1}, M_{2}\right\}$.

Now we construct $D_{1}^{\prime}$ from $D_{1}$ as follows: for each reversed edge $e$ in $D_{2}^{\prime}$, if $e=e_{i}$ for some $1 \leq i \leq t$, then we reverse the orientations of all edges of $P_{i}$ in $D_{1}$; otherwise, we reverse the orientation of $e$ in $D_{1}$. Finally, we obtain a desired modulo 3-orientation $D_{1}^{\prime}$ of $G$.

\subsubsection{Vector $S^{1}$-flows with rank two}

In this subsection, we will prove Theorem 1.5.7 for the case of vector flows with rank two.

Theorem 4.3.10. If a graph $G$ has a vector $S^{1}$-flow $(D, \Phi)$ with rank two, then $G$ admits an integer $3-N Z F$.

Proof. Note that $G$ is bridgeless since $G$ admits a vector $S^{1}$-flow. By Theorem 1.3.11, it is equivalent to show that $G$ admits a modulo 3-orientation. By Lemma 4.3.1-(2), we can assume that each vertex of $G$ is unsplittable. Note that we can further assume that $G$ has an odd- $k$-edge cut with $2<k<7$. Otherwise, by Theorem 1.5.1, $G$ admits an integer 3-NZF. Denote the balanced vector of the smallest odd-edge cut by

$$
\boldsymbol{\eta}=<\eta_{1}, \eta_{2}, \ldots, \eta_{b}>
$$


Thus, $\|\boldsymbol{\eta}\| \in\{3,5\}$. In either case, by Lemma 4.3.6-(1-a), there exists some $i$ such that $\left|\eta_{i}\right|=1$. Without lose of generality, assume $\left|\eta_{1}\right|=1$ and $\boldsymbol{\eta}=<\eta_{1}, \eta_{2}, \ldots, \eta_{a}, 0, \ldots, 0>$, where $a \leq b$ and $\eta_{i} \neq 0$ for each $1 \leq i \leq a$. We further assume $\eta_{i} \geq 0$ for each $i \leq b$, otherwise we reverse the orientations of all $\boldsymbol{\alpha}_{i}$-edges if $\eta_{i}<0$ and negate their flow values.

For each $\boldsymbol{\alpha}_{1}$-edge $u v$ directed from $u$ to $v$ in $D$, we subdivide this edge into a path of length 3 by inserting two new vertices $w_{1}$ and $w_{2}$. Denote this path by $u w_{1} w_{2} v$. Orient $w_{1} w_{2}$ from $w_{1}$ to $w_{2}$ and assign $w_{1} w_{2}$ with the vector $\boldsymbol{\alpha}_{1}$. Replace $w_{1} u$ with $\|\boldsymbol{\eta}\|-1$ multiple edges oriented from $w_{1}$ to $u$ and assign these $\|\boldsymbol{\eta}\|-1$ edges with vectors in $\left\{\boldsymbol{\alpha}_{2}, \ldots, \boldsymbol{\alpha}_{b}\right\}$ such that $w_{1}$ is balanced with the balanced vector $\boldsymbol{\eta}$. Similarly, replace $v w_{2}$ with $\|\boldsymbol{\eta}\|-1$ multiple edges oriented from $v$ to $w_{2}$ and assign these $\|\boldsymbol{\eta}\|-1$ edges with vectors in $\left\{\boldsymbol{\alpha}_{2}, \ldots, \boldsymbol{\alpha}_{b}\right\}$ such that $w_{2}$ is balanced with the balanced vector $-\boldsymbol{\eta}$. Denote the resulting graph by $G^{\prime}$. Note that $G^{\prime}$ admits a vector $S^{1}$-flow, say $\left(D^{\prime}, \boldsymbol{\Phi}^{\prime}\right)$, and has the same balanced vector space as $G$. Moreover, if $G^{\prime}$ has a modulo 3-orientation, then $G$ has a modulo 3-orientation. Similarly, by Lemma 4.3.1-(2), we can assume that each vertex in $G^{\prime}$ is unsplittable.

Now we choose a vector $\phi=<\phi_{1}, \phi_{2}, \ldots, \phi_{b}>$ of $S\left(\Phi^{\prime}\right)$ such that

(1) each coordinate $\phi_{i}$ is an integer and $\phi_{1}=0$;

(2) $\{\boldsymbol{\eta}, \boldsymbol{\phi}\}$ is a basis of $S\left(\boldsymbol{\Phi}^{\prime}\right)$;

(3) subject to (1) and (2), $\|\boldsymbol{\phi}\|$ is as small as possible.

Remark. Note that such $\phi$ does exist in $\left(D^{\prime}, \boldsymbol{\Phi}^{\prime}\right)$ because there are some vertices with the first coordinate equal to zero in their balanced vectors. Moreover, all those balanced vectors are linearly independent of $\boldsymbol{\eta}$.

Claim 16. For each vertex $v \in V\left(G^{\prime}\right)$, the balanced vector of $v$ is either $\boldsymbol{\eta}$ or $-\boldsymbol{\eta}$ or is an integer multiple of $\phi$.

Proof. If $v$ is incident with an $\boldsymbol{\alpha}_{1}$-edge, then by the definition of $G^{\prime}, \boldsymbol{\epsilon}(v)$ is either $\boldsymbol{\eta}$ or $-\boldsymbol{\eta}$. Otherwise, we have $\epsilon_{1}(v)=0$. Since $\{\boldsymbol{\eta}, \boldsymbol{\phi}\}$ is a basis of $S\left(\boldsymbol{\Phi}^{\prime}\right)$, we have $\boldsymbol{\epsilon}(v)=p \boldsymbol{\eta}+q \boldsymbol{\phi}$ 
for some rational numbers $p$ and $q$. Since $\epsilon_{1}(v)=\phi_{1}=0$ and $\eta_{1}=1$, we have $p=0$. Thus, $\boldsymbol{\epsilon}(v)=q \boldsymbol{\phi}$. Note that the greatest common divisor of nonzero coordinates in $\phi$ is 1 by the minimality of $\|\phi\|$. Since each $\phi_{i}$ and each $\epsilon_{i}(v)$ are integers, $q$ must be an integer.

Note that $\eta_{j}=0$ if $j \geq a+1$. Thus, if $\phi_{j}<0$ for some $j \geq a+1$, we may reverse the orientations of all $\boldsymbol{\alpha}_{j}$-edges and negate the flow values to make $\phi_{j}>0$ without affecting $\boldsymbol{\eta}$. Thus, we can assume $\phi_{j} \geq 0$ for each $j \geq a+1$. By relabeling $\boldsymbol{\alpha}_{i}$ 's, we may further assume $\eta_{2} \geq \cdots \geq \eta_{a}>0$ and $\phi_{a+1} \geq \cdots \geq \phi_{b} \geq 0$. In summary, we assume

$$
\boldsymbol{\eta}=<\eta_{1}, \ldots, \eta_{a}, 0, \ldots, 0>\quad \text { and } \quad \boldsymbol{\phi}=<\phi_{1}, \phi_{2}, \ldots, \phi_{b}>,
$$

which satisfy the following:

(1) $\eta_{1}=1,\|\boldsymbol{\eta}\| \in\{3,5\}$, and $\eta_{2} \geq \eta_{3} \geq \cdots \geq \eta_{a}>0$.

(2) $\phi_{1}=0$ and $\phi_{a+1} \geq \cdots \geq \phi_{b} \geq 0$.

Since $G^{\prime}$ is unsplittable, combined with Claim 16 and Lemma 4.3.3-(2) we can further split each vertex $v$ whose balanced vector is $q \phi$ into $|q|$ vertices such that the balanced vector of each new vertex is either $\phi$ or $-\phi$. Let $H$ denote the resulting graph. Thus, $H$ admits a vector $S^{1}$-flow by Lemma 4.3.1-(1), say $\left(D_{h}, \boldsymbol{\Phi}_{h}\right)$, such that the balanced vector of each vertex is in $\{ \pm \boldsymbol{\eta}, \pm \boldsymbol{\phi}\}$. Therefore, $V(H)$ can be partitioned into $V_{1}$ and $V_{2}$, where

$$
V_{1}=\{v \in V(H): \boldsymbol{\epsilon}(v)= \pm \boldsymbol{\eta}\} \quad \text { and } \quad V_{2}=\{v \in V(H): \boldsymbol{\epsilon}(v)= \pm \boldsymbol{\phi}\} \text {. }
$$

By Lemma 4.3.1-(2), it suffices to show that $H$ has a modulo 3-orientation. Suppose, in the following context, that $H$ does not have a modulo 3-orientation.

Claim 17. $\sum_{j=a+1}^{b} \phi_{j} \leq 1$ and $a \geq 4$. Moreover,

(1) $\phi_{a+1} \in\{0,1\}$ and $\phi_{a+2}=\cdots=\phi_{b}=0$.

(2) $\|\boldsymbol{\eta}\|=5$ and either $\boldsymbol{\eta}=<1,1,1,1,1,0, \ldots, 0>$ or $\boldsymbol{\eta}=<1,2,1,1,0, \ldots, 0>$. 
Proof. If $\sum_{j=a+1}^{b} \phi_{j} \geq 2$, then by Lemma 4.3.9-(1), $H$ has a modulo 3-orientation since $\sum_{i=1}^{a}\left|\eta_{i}\right| \leq 5$. This yields a contradiction. Thus, $\sum_{j=a+1}^{b} \phi_{j} \leq 1$ and thus $\phi_{a+1} \in\{0,1\}$, $\phi_{a+2}=\cdots=\phi_{b}=0$.

If $a \leq 3$, then $a=3$ since $\boldsymbol{\alpha}_{1}$ and $\boldsymbol{\alpha}_{2}$ are linearly independent. Since $\|\boldsymbol{\eta}\|=3$ or 5 , we have $\eta_{2}=\eta_{3}$. Denote $t=\eta_{2}$. Thus, $\boldsymbol{\alpha}_{1}+t \boldsymbol{\alpha}_{2}+t \boldsymbol{\alpha}_{3}=\mathbf{0}$. Since $\phi_{1}=0$, we have $\phi_{4} \neq 0$ and thus $\left|\phi_{4}\right|=1$. Now we have $\phi_{2} \boldsymbol{\alpha}_{2}+\phi_{3} \boldsymbol{\alpha}_{3}+\phi_{4} \boldsymbol{\alpha}_{4}=\mathbf{0}$ where $\left|\phi_{4}\right|=1$, a contradiction to Lemma 4.3.6-(2). Therefore, $a \geq 4$. This implies $\|\boldsymbol{\eta}\|=5$ and either $\boldsymbol{\eta}=<1,1,1,1,1,0, \ldots, 0>$ or $\boldsymbol{\eta}=<1,2,1,1,0, \ldots, 0>$.

Claim 18. $\|\phi\| \geq 5$.

Proof. Suppose $\|\phi\|<5$. By Lemma 4.3.6-(1), $\|\phi\|=3$ and $\phi$ has exactly three coordinates with absolute value equal to 1 . If there exist $i$ and $j$ such that $\phi_{i}=\phi_{j}=t \in\{-1,1\}$ and $2 \leq i<j \leq a$, then it is easy to see that $(\boldsymbol{\eta}-t \boldsymbol{\phi})$ has length either 2 or 4 , a contradiction to Lemma 4.3.6-(1-b). Otherwise, by Claim 17-(1), $\phi_{a+1} \leq 1$ and thus $\phi_{a+1}=1$, and there exist $i$ and $j$ such that $\phi_{i}=-\phi_{j} \in\{-1,1\}$ and $2 \leq i<j \leq a$. By Claim 17-(2), $\eta_{j}=1$. Now one can obtain a obtain a modulo 3-orientation of $H$ by reversing the orientations of all $\boldsymbol{\alpha}_{k}$-edges for each $k \in\{j, a+1\}$ with $\phi_{k} \neq \phi_{i}$. This is a contradiction.

Claim 19. $\eta_{j}=\left|\phi_{j}\right|=1$ for some $j \in\{2,3,4,5\}$ and $\phi_{a+1}=1$.

Proof. Since $H$ does not have a modulo 3-orientation, it follows from Lemma 4.3.9-(2) that for each $j=2, \ldots, a$, if $\eta_{j}=1$, then $\left|\phi_{j}\right| \leq 1$. Suppose $\left|\phi_{j}\right|=0$ for each $j$ with $\eta_{j}=1$.

By Claim 17-(2), either $\boldsymbol{\eta}=<1,1,1,1,1,0, \ldots, 0>$ or $\boldsymbol{\eta}=<1,2,1,1,0, \ldots, 0>$. Thus, $\phi_{3}=\cdots=\phi_{a}=0$. By Claim 17-(1), $\phi_{a+1} \leq 1$ and $\phi_{j}=0$ for each $j \geq a+2$. Thus, $\phi_{2} \boldsymbol{\alpha}_{2}+\phi_{a} \boldsymbol{\alpha}_{a+1}=\mathbf{0}$. Since $\boldsymbol{\alpha}_{2}$ and $\boldsymbol{\alpha}_{a+1}$ are linearly independent, $\phi_{2}=\phi_{a+1}=0$. This implies that $\phi$ is a zero vector, a contradiction to the choice of $\phi$. Therefore, there must be some $j \in\{2,3,4,5\}$ such that $\eta_{j}=\left|\phi_{j}\right|=1$.

By Claim 17-(1), if $\phi_{a+1} \neq 1$, then $\phi_{a+1}=0$ and thus $\phi_{i}=0$ for each $i \geq a+1$. By Claim 18, $\sum_{i=1}^{a}\left|\phi_{i}\right| \geq 5=\sum_{i=1}^{a} \eta_{i}, \phi_{1}=0$, and there must exist some $j \in\{2, \ldots, a\}$ 
such that $1 \leq \eta_{j}<\left|\phi_{j}\right|$. Since $\eta_{i}=1$ implies $\left|\phi_{i}\right| \leq 1$, we have $\eta_{j} \geq 2$ and $\left|\phi_{j}\right| \geq 3$. By Claim $17, \boldsymbol{\eta}=<1,2,1,1,0, \ldots, 0>$ and thus $j=2$. Now we have

$$
\phi_{2} \boldsymbol{\alpha}_{2}+\phi_{3} \boldsymbol{\alpha}_{3}+\phi_{4} \boldsymbol{\alpha}_{4}=\mathbf{0},
$$

where $\left|\phi_{2}\right| \geq 3$ and $\left|\phi_{3}\right|,\left|\phi_{4}\right| \leq 1$, a contradiction to Lemma 4.3.6-(1-a). This proves $\phi_{a+1}=1$.

\section{The final step.}

If $\sum_{i=2}^{b} \phi_{i} \equiv 0(\bmod 3)$, then one can obtain a modulo 3-orientation of $H$ by reversing the orientations of all edges of $E_{1}(H)$ in $D_{h}$ since $\phi_{1}=0$, a contradiction.

If $\sum_{i=2}^{b} \phi_{i} \equiv 2(\bmod 3)$, then one can obtain a modulo 3-orientation of $H$ by reversing the orientations of all edges of $E_{1}(H) \cup E_{a+1}(H)$ in $D_{h}$ since $\phi_{a+1}=\eta_{1}=1$ and $\eta_{a+1}=$ $\phi_{1}=0$, a contradiction again. Therefore, $\sum_{i=2}^{b} \phi_{i} \equiv 1(\bmod 3)$.

By Claim 19, $\eta_{j}=\left|\phi_{j}\right|=1$ for some $j \leq a$. Note that $\phi_{a+1}=1$ and $\eta_{a+1}=0$. If $\phi_{j}=1$, then we reverse the orientations of all edges in $E_{j}(H) \cup E_{a+1}(H)$ in $D_{h}$. and otherwise reverse the orientations of all edges of $E_{j}(H)$ in $D_{h}$. In either case, the resulting orientation is a modulo 3 -orientation of $H$, a contradiction. This completes the proof of Theorem 4.3.10.

\subsection{Proof of Theorem 1.5.9}

Proof. We only need to prove the sufficiency by Theorem 1.5.4. Suppose that $G$ admits a vector $S^{1}$-flow $(D, \Phi)$. Let $v$ be a vertex of $G$ of degree three. Without loss of generality, we assume $E^{-}(v)=\emptyset$. Denote $\left\{\boldsymbol{\alpha}_{1}, \boldsymbol{\alpha}_{2}, \boldsymbol{\alpha}_{3}\right\}=\left\{\boldsymbol{\Phi}(e): e \in E^{+}(v)\right\}$. Thus,

$$
\boldsymbol{\alpha}_{1}+\boldsymbol{\alpha}_{2}+\boldsymbol{\alpha}_{3}=\mathbf{0}
$$

We further assume that no edges of $G$ have flow value $-\boldsymbol{\alpha}_{i}$ for each $i=1,2,3$ since otherwise we can reverse the orientations of all $\left(-\boldsymbol{\alpha}_{i}\right)$-edges and negate their flow values. By Equation (4.3), we have the following claim. 
Claim 20. Let $\boldsymbol{\beta}=t_{1} \boldsymbol{\alpha}+t_{2} \boldsymbol{\alpha}_{2}+t_{3} \boldsymbol{\alpha}_{2}$ where $t_{1}, t_{2}$, and $t_{3}$ are integers. Then the following two statements are true.

(1) If $\boldsymbol{\beta}=\mathbf{0}$, then $t_{1}=t_{2}=t_{3}$.

(2) If $|\boldsymbol{\beta}|=1$, then $\boldsymbol{\beta} \in\left\{ \pm \boldsymbol{\alpha}_{1}, \pm \boldsymbol{\alpha}_{2}, \pm \boldsymbol{\alpha}_{3}\right\}$.

Proof. Without loss of generality, we assume $t_{1} \leq t_{2} \leq t_{3}$. By eliminating $\boldsymbol{\alpha}_{1}$ using Equation (4.3), $\boldsymbol{\beta}=\left(t_{2}-t_{1}\right) \boldsymbol{\alpha}_{2}+\left(t_{3}-t_{1}\right) \boldsymbol{\alpha}_{3}$

(1) If $\boldsymbol{\beta}=\mathbf{0}$, then $\left(t_{2}-t_{1}\right) \boldsymbol{\alpha}_{2}+\left(t_{3}-t_{1}\right) \boldsymbol{\alpha}_{3}=\mathbf{0}$. Since $\boldsymbol{\alpha}_{2}$ and $\boldsymbol{\alpha}_{3}$ are linearly independent, $t_{2}-t_{1}=t_{3}-t_{1}=0$ implying $t_{1}=t_{2}=t_{3}$.

(2) If $|\boldsymbol{\beta}|=1$, suppose to the contrary $\boldsymbol{\beta} \notin\left\{ \pm \boldsymbol{\alpha}_{1}, \pm \boldsymbol{\alpha}_{2}, \pm \boldsymbol{\alpha}_{3}\right\}$. Then $\boldsymbol{\beta}$ is linearly independent of $\boldsymbol{\alpha}_{2}$ and $\boldsymbol{\alpha}_{3}$. Thus, $t_{2}-t_{1} \neq 0$ and $t_{3}-t_{1} \neq 0$. By Lemma 4.3.6-(1-a), we have $\left|t_{2}-t_{1}\right|=\left|t_{3}-t_{1}\right|$. Since $t_{1} \leq t_{2} \leq t_{3}$, we have $t_{2}-t_{1}=t_{3}-t_{1}$. Thus, $\boldsymbol{\beta}=\left(t_{2}-t_{1}\right)\left(\boldsymbol{\alpha}_{2}+\boldsymbol{\alpha}_{3}\right)=-\left(t_{2}-t_{1}\right) \boldsymbol{\alpha}_{1}$. Since $|\boldsymbol{\beta}|=\left|\boldsymbol{\alpha}_{1}\right|=1$, we have $t_{2}-t_{1}=|\boldsymbol{\beta}|=1$ and $\boldsymbol{\beta}=-\boldsymbol{\alpha}_{1}$. This contradiction implies $\boldsymbol{\beta} \in\left\{ \pm \boldsymbol{\alpha}_{1}, \pm \boldsymbol{\alpha}_{2}, \pm \boldsymbol{\alpha}_{3}\right\}$.

Claim 21. For each vertex $u$ of degree three, $\{\boldsymbol{\Phi}(e): e \in E(u)\}=\left\{\boldsymbol{\alpha}_{1}, \boldsymbol{\alpha}_{2}, \boldsymbol{\alpha}_{3}\right\}$.

Proof. Since $G\left[V_{3}\right]$ is connected, it suffices to show that, for each vertex $u$ of degree three and adjacent to $v,\{\Phi(e): e \in E(u)\}=\left\{\boldsymbol{\alpha}_{1}, \boldsymbol{\alpha}_{2}, \boldsymbol{\alpha}_{3}\right\}$. Without loss of generality, assume $\boldsymbol{\Phi}(v u)=\boldsymbol{\alpha}_{3}$. Since $\operatorname{deg}(u)=3$, there are two other vectors $\boldsymbol{\beta}_{1}, \boldsymbol{\beta}_{2} \in S^{1}$ such that

$$
\boldsymbol{\beta}_{1}+\boldsymbol{\beta}_{2}-\boldsymbol{\alpha}_{3}=\mathbf{0}
$$

Adding Equation (4.3) to Equation (4.4), we have

$$
\boldsymbol{\alpha}_{1}+\boldsymbol{\alpha}_{2}+\boldsymbol{\beta}_{1}+\boldsymbol{\beta}_{2}=\mathbf{0}
$$

Since all vectors in $S^{1}$ are unit vectors, the vectors $\boldsymbol{\alpha}_{1}, \boldsymbol{\alpha}_{2}, \boldsymbol{\beta}_{1}$, and $\boldsymbol{\beta}_{2}$ form a parallelogram in $\mathbb{R}^{2}$. Thus, $\left\{\boldsymbol{\beta}_{1}, \boldsymbol{\beta}_{2}\right\}=\left\{-\boldsymbol{\alpha}_{1},-\boldsymbol{\alpha}_{2}\right\}$ and $E^{+}(u)=\emptyset$ since there is no edge $e$ in $G$ having $\boldsymbol{\Phi}(e)=-\boldsymbol{\alpha}_{i}$ for each $i=1,2,3$. Therefore, $\{\boldsymbol{\Phi}(e): e \in E(u)\}=\left\{\boldsymbol{\alpha}_{1}, \boldsymbol{\alpha}_{2}, \boldsymbol{\alpha}_{3}\right\}$.

Claim 22. For each edge e of $G, \boldsymbol{\Phi}(e) \in\left\{\boldsymbol{\alpha}_{1}, \boldsymbol{\alpha}_{2}, \boldsymbol{\alpha}_{3}\right\}$. 
Proof. Let $E^{\prime}=\left\{e \in E(G): \boldsymbol{\Phi}(e) \neq \boldsymbol{\alpha}_{i}\right.$, for each $\left.i=1,2,3\right\}$ and $G^{\prime}=G\left[E^{\prime}\right]$. We only need to show $E^{\prime}=\emptyset$. Suppose to the contrary $E^{\prime} \neq \emptyset$.

By Claim 21, $G^{\prime}$ is a subgraph of $G-V_{3}$ and thus is acyclic. If $E^{\prime} \neq \emptyset$, then there exists a vertex $w$ of degree one in $G^{\prime}$ since $G^{\prime}$ is acyclic. Let $w z$ be the edge in $E^{\prime}$ with flow value $\boldsymbol{\beta}$. Then except $w z$, the flow value of each other edge incident with $w$ is $\boldsymbol{\alpha}_{i}$ for some $i \in\{1,2,3\}$. Without lose of generality, we assume that $w z$ is oriented from $w$ to $z$. Then the balanced vector of $w$ is

$$
\boldsymbol{\epsilon}(w)=\boldsymbol{\beta}+r_{1} \boldsymbol{\alpha}_{1}+r_{2} \boldsymbol{\alpha}_{2}+r_{3} \boldsymbol{\alpha}_{3}=\mathbf{0},
$$

where $r_{1}, r_{2}$, and $r_{3}$ are integers. Thus, $\boldsymbol{\beta}=-r_{1} \boldsymbol{\alpha}_{1}-r_{2} \boldsymbol{\alpha}_{2}-r_{3} \boldsymbol{\alpha}_{3}$. By Claim 20, $\boldsymbol{\beta} \in\left\{\boldsymbol{\alpha}_{1}, \boldsymbol{\alpha}_{2}, \boldsymbol{\alpha}_{3}\right\}$ because $|\boldsymbol{\beta}|=1$ and no edge has flow value $-\boldsymbol{\alpha}_{i}$ for each $i=1, \ldots, k$. This contradicts the assumption that $\boldsymbol{\beta} \notin\left\{\boldsymbol{\alpha}_{1}, \boldsymbol{\alpha}_{2}, \boldsymbol{\alpha}_{3}\right\}$. Therefore, $E^{\prime}=\emptyset$.

Claim 22 implies that the balanced vector of each vertex $v$ is $\boldsymbol{\epsilon}(v)=t_{1} \boldsymbol{\alpha}_{1}+t_{2} \boldsymbol{\alpha}_{2}+$ $t_{3} \boldsymbol{\alpha}_{3}=\mathbf{0}$ for some integers $t_{1}, t_{2}, t_{3}$. By Claim 20, $t_{1}=t_{2}=t_{3}$ and thus $\boldsymbol{\epsilon}(v)=t_{1}\left(\boldsymbol{\alpha}_{1}+\right.$ $\left.\boldsymbol{\alpha}_{2}+\boldsymbol{\alpha}_{3}\right)$. This implies that the rank of $S(\boldsymbol{\Phi})$ is one. Therefore $G$ admits an integer 3-NZF by Theorem 1.5.7. 


\section{Chapter 5}

\section{Even Factors of Graphs}

In this chapter, our goal is to show that if a graph $G$ has an even factor $F$, then it has an even factor $H$ such that

$$
|E(H)| \geq \frac{4}{7}(|E(G)|+1)+\frac{1}{7}\left|V_{2}(G)\right| .
$$

Without loss of generality, all graphs considered in our proof are connected, since the inequality being considered as above is obviously additive over connected components.

Proof of Theorem 1.6.5. Let $(G, F)$ be a contra pair to Theorem 1.6.5 such that

(1) $|E(G)|$ is minimized;

(2) subject to $(1), m(G)=\sum_{v \in V_{\geq 3}(G)}(\operatorname{deg}(v)-3)$ is minimized;

(3) subject to (1) and (2), $|E(F)|$ is maximized;

(4) subject to (1)-(3), the number of components of $F$ is as small as possible.

Claim 23. $V_{2}(G)$ is an independent subset of $V(G)$.

Proof. Suppose that there are two vertices $u, v \in V_{2}(G)$ such that $u v \in E(G)$. Let $G^{\prime}$ (and $F^{\prime}$ ) be the graph obtained from $G$ (and $F$ ) by identifying $u$ and $v$ into a new vertex 
$w$ and deleting the resulting loop. Clearly, $\left|E\left(G^{\prime}\right)\right|<|E(G)|, w \in V_{2}\left(F^{\prime}\right)$, and $F^{\prime}$ is an even factor of $G^{\prime}$. By assumption (1), $G^{\prime}$ has an even factor $H^{\prime}$ such that

$$
\left|E\left(H^{\prime}\right)\right| \geq \frac{4}{7}\left(\left|E\left(G^{\prime}\right)\right|+1\right)+\frac{1}{7}\left|V_{2}\left(G^{\prime}\right)\right| .
$$

Let $H$ be the subgraph of $G$ induced by $\left(E\left(H^{\prime}\right)-E_{H^{\prime}}(w)\right) \cup E(u) \cup E(v)$. Since $w \in V_{2}\left(F^{\prime}\right)$, $H$ is an even factor of $G$ with $|E(H)|=\left|E\left(H^{\prime}\right)\right|+1$. Note that $|E(G)|=\left|E\left(G^{\prime}\right)\right|+1$ and $\left|V_{2}(G)\right|=\left|V_{2}\left(G^{\prime}\right)\right|+1$. Hence,

$$
\begin{aligned}
|E(H)| & \geq\left(\frac{4}{7}\left(\left|E\left(G^{\prime}\right)\right|+1\right)+\frac{1}{7}\left|V_{2}\left(G^{\prime}\right)\right|\right)+1 \\
& =\frac{4}{7}|E(G)|+\frac{1}{7}\left(\left|V_{2}(G)\right|-1\right)+1 \\
& =\frac{4}{7}(|E(G)|+1)+\frac{1}{7}\left|V_{2}(G)\right|+\frac{2}{7} \\
& >\frac{4}{7}(|E(G)|+1)+\frac{1}{7}\left|V_{2}(G)\right|
\end{aligned}
$$

This contradicts the choice of $(G, F)$.

Claim 24. $F$ is a 2-factor of $G$.

Proof. Suppose that one component $I$ of $F$ contains a vertex $v$ such that $\operatorname{deg}_{F}(v)=2 t \geq 4$. Since each component of $F$ is an eulerian subgraph of $G$, we have an eulerian tour $T$ from $I$. We split $v$ into $t$ vertices of degree 2, each of which is incident with two consecutive edges in $T$. Denote the resulting graphs by $F^{\prime}$ (from $F$ ) and $G^{\prime}$ (from $G$ ), respectively. By assumption (2), $\left(G^{\prime}, F^{\prime}\right)$ is not a contra pair since $\left|E\left(G^{\prime}\right)\right|=|E(G)|$ and $m\left(G^{\prime}\right)<m(G)$. Thus, $G^{\prime}$ has an even factor $H^{\prime}$ such that

$$
\left|E\left(H^{\prime}\right)\right| \geq \frac{4}{7}\left(\left|E\left(G^{\prime}\right)\right|+1\right)+\frac{1}{7}\left|V_{2}\left(G^{\prime}\right)\right| .
$$

Note that $\left|V_{2}\left(G^{\prime}\right)\right|=\left|V_{2}(G)\right|+t>\left|V_{2}(G)\right|$. Let $H$ be the subgraph of $G$ induced by $E\left(H^{\prime}\right)$, then $H$ is an even factor of $G$ with $|E(H)|=\left|E\left(H^{\prime}\right)\right|$. Furthermore,

$$
\begin{aligned}
|E(H)| & \geq \frac{4}{7}\left(\left|E\left(G^{\prime}\right)\right|+1\right)+\frac{1}{7}\left|V_{2}\left(G^{\prime}\right)\right| \\
& =\frac{4}{7}(|E(G)|+1)+\frac{1}{7}\left(\left|V_{2}(G)\right|+t\right) \\
& >\frac{4}{7}(|E(G)|+1)+\frac{1}{7}\left|V_{2}(G)\right|
\end{aligned}
$$

This contradicts the choice of $(G, F)$. 


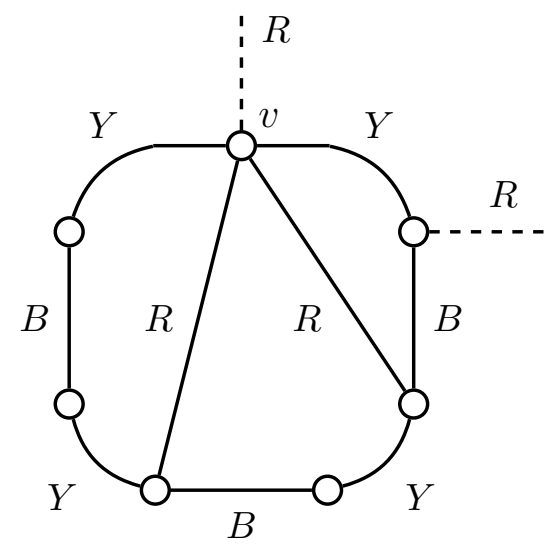

Figure 5.1: The $\{B, Y, R\}$-edge-coloring around an odd circuit of $F$, and the selection of the vertex $v$

\section{$5.1\{\mathrm{~B}, \mathrm{Y}, \mathrm{R}\}$-edge-coloring of $G$}

In this part, we follow an edge-coloring introduced by Chen and Fan [6] and define an $\{B, Y, R\}$-edge-coloring (*) of $G$ as follows.

For a maximum matching $B$ of $F$, let $Y=E(F)-B$ and $R=E(G)-E(F)$. For simplicity, we use $R, B$, and $Y$ to denote the subgraphs induced by $E(R), E(B)$, and $E(Y)$, respectively. Note that $F$ is a union of disjoint circuits by Claim 24. We will pick a particular maximum matching $B$ out of $F$ by choosing, for each odd circuit $C$ of $F$, the vertex $v$ not incident with any member of $E(C) \cap B$ according to the following rules. See Figure 5.1 for an illustration.

(a) if $C$ is an odd circuit of $F$ and $F$ has more than one component, then let $v$ be a vertex of $C$ such that $\operatorname{deg}_{D}(v)$ is as large as possible in $D=G[V(C)]$ and $v$ is incident with both $Y$-edges in $C$;

(b) subject to (a) $\operatorname{deg}_{R}(v)$ is as large as possible.

Denote the collection of such vertices by $V_{0}(G)$. An edge $e$ is called an $B$-edge ( $Y$-edge, $R$-edge, respectively) if $e \in B$ ( $e \in Y, e \in R$, respectively). 
Claim 25. $R \cup B$ is a spanning subgraph of $G$.

Proof. Suppose that $G$ has a vertex $v \in V(G)-V(R \cup B)$. Then $v$ is incident with only $Y$-edges and therefore $v \in V_{0}(G)$. By Claim 24, $\operatorname{deg}(v)=2$ and $v$ is incident with precisely two $Y$-edges. By the definition of $V_{0}(G)$, there is an odd component $C$ of $F$ such that $v \in V(C)$. By rule (b) in (*), each vertex of $C$ has degree 2 in $G$. This implies $G=C$, a contradiction to the choice of $(G, F)$.

Claim 26. Each circuit in $R \cup B$ is alternately $\{R, B\}$-edge-colored, and any two circuits are disjoint.

Proof. Suppose that $Z$ is a circuit of $R \cup B$ that is not alternately $\{R, B\}$-edge-colored. Since $B$ is a matching of $F, Z \triangle F$ is an even factor larger than $F$, a contradiction to assumption (3).

Suppose $Z_{1}$ and $Z_{2}$ are two non-disjoint circuits in $R \cup B$ and denote $K=Z_{1} \cup Z_{2}$. Then $K$ contains a vertex $v$ of degree at least 3 . Note that any vertex of degree 4 in $K$ must be incident with two $R$-edges and two $B$-edges contradicting that $B$ is a matching. Thus, $\operatorname{deg}_{K}(v)=3$ and each vertex of degree 3 is incident with one $B$-edge and two $R$-edges (one is in $Z_{1}$ and one is in $Z_{2}$ ). This implies that $Z_{1} \triangle Z_{2}$ contains a circuit of $R \cup B$ in which two $R$-edges are adjacent to each other. This contradicts that each circuit in $R \cup B$ is alternately $\{R, B\}$-edge-colored.

Claim 27. If $Z$ is a circuit of length $2 \mu$ of $R \cup B$, then the number of components of $F$ intersecting with $Z$ is at most $\max \{\mu-1,1\}$.

Proof. Suppose that there is a circuit $Z$ of $R \cup B$ of length $2 \mu$ intersecting with $\mu(\mu \geq 2)$ components of $F$. By Claim 26, the circuits in $R \cup B$ are alternately $\{R, B\}$-edge-colored and pairwise disjoint. Thus, $F \triangle Z$ is an even factor of $G$. Since the $(2 \mu)$-circuit $Z$ intersects with a set $\mathcal{X}$ of $\mu$ distinct components (circuits) of $F,|E(Z) \cap E(C)|=1$ for every $C \in \mathcal{X}$ and therefore $\left(\bigcup_{C \in \mathcal{X}} C\right) \triangle Z$ is a single circuit. Hence, $F \triangle Z$ has $(\mu-1)$ components less than $F$, a contradiction to assumption (4). 


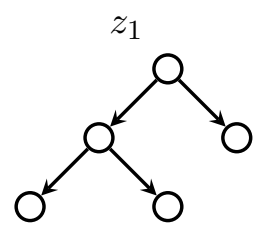

$T_{1}$

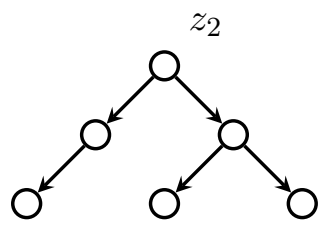

$T_{2}$

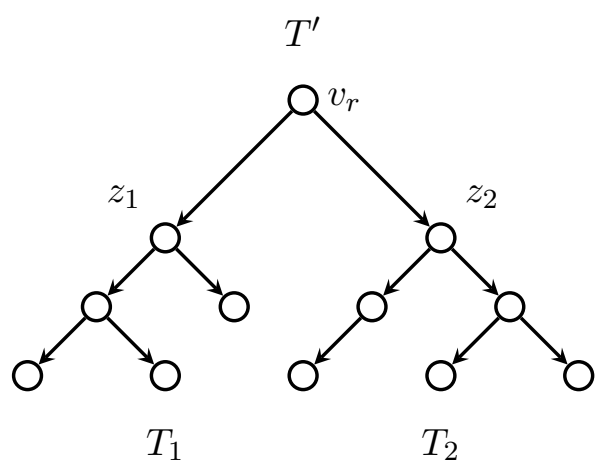

Figure 5.2: The forest $T_{1} \cup T_{2}=(R \cup B) / \mathcal{Z}$ and its extension $T^{\prime}$ (a rooted tree)

\section{$5.2 \quad Q$-subgraphs in $R \cup R^{*} \cup B$}

In Claims 25, 26, and 27, the structure of circuits contained in $G[R \cup B]$ has been studied. Note that not every $B$-edge is contained in some circuit of $G[R \cup B]$. Denote the collection of such $B$-edges by $B_{0}$ and call each of its elements an $B_{0}$-edge. By Claim 25 , each vertex of degree 2 must be incident with an $B_{0}$-edge. By Claim 23, none of these $B_{0}$-edges is incident with two vertices of degree 2. Thus, $\left|B_{0}\right| \geq\left|V_{2}\right|$.

Let $\mathcal{Z}=\left\{Z_{1}, \cdots, Z_{t}\right\}$ be the collection of all circuits in $R \cup B$. Let $t_{k}$ be the number of $k$-circuits of $\mathcal{Z}$. Let $T$ be the graph obtained from $R \cup B$ by contracting each $Z_{i}$ into a vertex. By Claim 26, the circuits in $\mathcal{Z}$ are pairwise disjoint and therefore $T$ is acyclic. Let $\left\{T_{1}, \cdots, T_{a}\right\}$ be all components of $T$ and assign an orientation to each $T_{i}$ as a rooted tree with the root $z_{i}$ ( $z_{i}$ is selected arbitrarily). Then we further extend the rooted forest $T_{1} \cup \cdots \cup T_{a}$ to be a rooted tree $T^{\prime}$ as follows: add a new vertex $v_{r}$ as the root of $T^{\prime}$ and add a new arc $\boldsymbol{v}_{\boldsymbol{r}} \boldsymbol{z}_{\boldsymbol{i}}\left(z_{i}\right.$ is the root of $\left.T_{i}\right)$ for each $1 \leq i \leq a$. See Figure 5.2 for an illustration. Denote $R^{*}=\left\{v_{r} z_{i}: 1 \leq i \leq a\right\}$.

Finally, we construct a tree $T^{*}$ from $T^{\prime}$ by further contracting all remaining $B$-edges in $T^{\prime}$. Note that all those $B$-edges are not contained in any circuit of $R \cup B$ and therefore are $B_{0}$-edges. In $T^{*}$, each edge is an $\left(R \cup R^{*}\right)$-edge and each vertex except $v_{r}$ is incident with a unique incoming arc. Now we can classify the vertex set $V\left(T^{*}\right)-\left\{v_{r}\right\}$ as follows. 
(1) A vertex $x$ is a Type I vertex if it is contracted from a circuit of $\mathcal{Z}$ in $R \cup B$.

(2) A vertex $x$ is a Type II vertex if it is contracted from an $B_{0}$-edge in $T^{\prime}$.

(3) A vertex $x$ is a Type III vertex if it is an original vertex in $G$. In this case, $x$ is not incident with any $B$-edge. Thus, $x \in V_{0}$.

For any vertex $x \in V\left(T^{*}\right)-\left\{v_{r}\right\}$, let $\boldsymbol{y} \boldsymbol{x}$ be the unique arc towards $x$ in $T^{*}$, and let $y u$ be the edge of $G$ corresponding to $\boldsymbol{y} \boldsymbol{x}$ in $T^{*}$. In $R \cup R^{*} \cup B$, we define the corresponding $Q$-subgraphs as follows. See Figure 5.3 for an illustration.

(1) If $x$ is Type I, let $Z \in \mathcal{Z}$ be the circuit contracted into $x$ in $T^{*}$. The subgraph induced by the circuit $Z$ and the edge $y u$ is called a Type I $Q$-subgraph. (A Type I $Q$-subgraph looks like a tadpole with the "body" $Z$ and the "trail" $y u$.)

(2) If $x$ is Type II, let $u v$ be the $B_{0}$-edge contracted into $x$ in $T^{*}$. The subgraph induced by the edges $u v$ and $y u$ is called a Type II $Q$-subgraph. (A Type II $Q$-subgraph is a path of length 3 , not looking like a tadpole at all. However, we still call the $B_{0}$-edge $u v$ the "body" and the edge $y u$ the "tail".)

(3) If $x$ is Type III, then $x=u$ remains as a vertex of $R \cup R^{*} \cup B$. The subgraph induced by the edge $y x$ is called a Type III $Q$-subgraph. (A Type III $Q$-subgraph is a single edge $y x$. Similarly, we call the single vertex $x$ the "body" and the edge $y x$ the "tail".)

Denote the body and the tail of each $Q$-subgraph $Q_{i}$ by $b\left(Q_{i}\right)$ and $t\left(Q_{i}\right)$, respectively. Note that the collection of all $Q$-subgraphs, denoted by $\mathcal{Q}$, is an edge partition of $R \cup R^{*} \cup B$.

Let $\mathcal{Q}_{I}\left(\mathcal{Q}_{I I}, \mathcal{Q}_{I I I}\right.$, respectively) be the collection of all Type I (Type II, Type III, respectively) $\mathcal{Q}$-subgraphs. Then

$$
\mathcal{Q}=\mathcal{Q}_{I} \cup \mathcal{Q}_{I I} \cup \mathcal{Q}_{I I}, \quad\left|\mathcal{Q}_{I I}\right|=\left|B_{0}\right|, \quad \text { and } \quad\left|\mathcal{Q}_{I I I}\right|=\left|V_{0}(G)\right|
$$




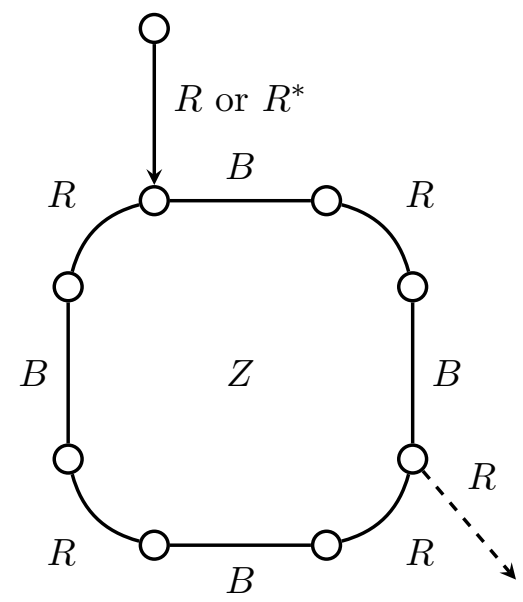

Type I.

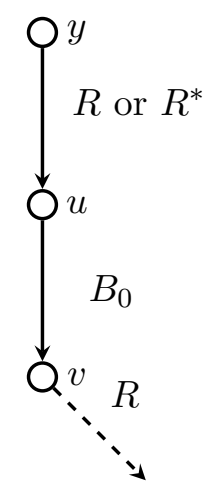

Type II.

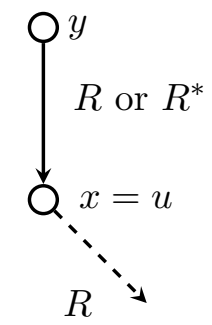

Type III.

Figure 5.3: Three Types of $Q$-subgraphs

\subsection{Charge and Discharge}

In $R \cup R^{*} \cup B$, assign each vertex $v$ of $G$ with initial charge $h_{V}(v)=1$ and $v_{r}$ with initial charge $h_{V}\left(v_{r}\right)=0$; assign each edge $e \in R \cup R^{*}$ with initial charge $h_{E}(e)=1$ and each edge $e^{\prime} \in B$ with initial charge $h_{E}\left(e^{\prime}\right)=0$, respectively. Then we have equations as follows.

\section{Claim 28.}

$$
\begin{aligned}
& n=|V(G)|=\sum_{v \in V(G)} h_{V}(v)=\sum_{Q_{i} \in \mathcal{Q}} h_{V}\left(Q_{i}\right) \\
& |R|+\left|R^{*}\right|=\sum_{e \in R \cup R^{*}} h_{E}(e)=\sum_{Q_{i} \in \mathcal{Q}} h_{E}\left(Q_{i}\right)
\end{aligned}
$$

where $h_{V}\left(Q_{i}\right)=\sum_{v \in b\left(Q_{i}\right)} h_{V}(v)$ and $h_{E}\left(Q_{i}\right)=\sum_{e \in E\left(Q_{i}\right)} h_{E}(e)$ for each $Q_{i} \in \mathcal{Q}$.

Proof. The proof of Equation (5.2) is trivial. The Equation (5.1) follows from Claim 25 that $R \cup B$ is a spanning graph of $G$. 
Now we define a ratio to each $Q$-subgraph $Q_{i}$ as follows:

$$
r\left(Q_{i}\right)=\frac{h_{V}\left(Q_{i}\right)}{h_{V}\left(Q_{i}\right)+h_{E}\left(Q_{i}\right)} .
$$

Then we have the following observations.

(1) For each $Q_{i} \in \mathcal{Q}_{I}$, it follows from Claim 26 that the body $b\left(Q_{i}\right)$ is an alternately $\{R, B\}$-edge-colored circuit $Z$ of $\mathcal{Z}$. If $|Z|=2 \mu$, then $r\left(Q_{i}\right)=\frac{2 \mu}{2 \mu+(\mu+1)}$ with $\mu \geq 2$. This implies $r\left(Q_{i}\right) \geq \frac{4}{7}$ since it is an increasing function on $\mu$.

(2) For each $Q_{i} \in \mathcal{Q}_{I I}$, the body $b\left(Q_{i}\right)$ is a single $B_{0}$-edge. Thus, $r\left(Q_{i}\right)=\frac{2}{3}\left(>\frac{4}{7}\right)$.

(3) For each $Q_{i} \in \mathcal{Q}_{I I I}$, the body $b\left(Q_{i}\right)$ is a single vertex. Thus, $r\left(Q_{i}\right)=\frac{1}{2}\left(<\frac{4}{7}\right)$.

To increase the ratio of $Q_{i} \in \mathcal{Q}_{I I I}$ to $\frac{4}{7}$, we either take some charge from a Type I $Q$-subgraph whose body is a $(\geq 6)$-circuit of $\mathcal{Z}$, or take some charge from a Type II $Q$-subgraph.

The following result guarantees that each $Q_{i} \in \mathcal{Q}_{I I I}$ can take some charge from an $Q$-subgraph in $\mathcal{Q}_{I} \cup \mathcal{Q}_{I I}$.

Claim 29. Every odd circuit $C$ of $F$ either contains at least one $B_{0}$-edge, or intersects with some $(\geq 6)$-circuit $Z_{i}$ of $R \cup B$.

Proof. Suppose that there is an odd circuit $C$ of $F$ such that each $B$-edge of $C$ is contained in a 4-circuit of $R \cup B$. By Claim 27, each of those 4-circuits intersects with $C$ only, no other components of $F$. Denote $G[V(C)]=K$. By rule (a) in (*), the minimum degree of $K$ is at least three. Note that $|V(K)|=|E(C)|$ is an odd integer. Thus, $|E(K)|>\frac{3}{2}|V(K)|=\frac{3}{2}|E(C)|$. By Theorem 1.6.1, $K$ has an even factor $D$ such that $|E(D)| \geq \frac{2}{3}|E(K)|>|E(C)|$. Thus, $D \cup(F-C)$ is an even factor of $G$ larger than $F$, a contradiction.

The Discharging Rule: Let $C$ be an odd circuit of $F$ with $x \in V(C) \cap V_{0}(G)$. We redistribute the charge as follows. 
(R1) If $C$ intersects with some $(\geq 6)$-circuit $Z$ of $\mathcal{Z}$, then $x$ takes $\frac{1}{3}$ from some $v \in$ $V(C) \cap V(Z)$.

(R2) Otherwise by Claim 29, $C$ contains at least one $B_{0}$-edge $u v$. Let $x$ take $\frac{1}{3}$ from $u$.

Now we have the following two observations.

Claim 30. Both Equations (5.1) and (5.2) remain the same after redistributing the charge.

Claim 31. The ratio of any Type III Q-subgraph is $\frac{4}{7}$ now.

For each $Q_{i} \in \mathcal{Q}_{I}$, the body $b\left(Q_{i}\right)$ is a circuit $Z$ of $R \cup B$. We define $l_{Q_{i}}$ to be the length of $Z$, and $k_{Q_{i}}$ to be the number of times of being discharged of $Q_{i}$. Then we have the following result.

Claim 32. For each $Q_{i} \in \mathcal{Q}_{I}$,

$$
h_{V}\left(Q_{i}\right)=\frac{4}{7}\left(h_{V}\left(Q_{i}\right)+h_{E}\left(Q_{i}\right)\right)+\frac{1}{7}\left(l_{Q_{i}}-k_{Q_{i}}-4\right) .
$$

Moreover, $r\left(Q_{i}\right) \geq \frac{4}{7}$ if $k_{Q_{i}} \leq l_{Q_{i}}-4$.

Proof. Since $Q_{i}$ is discharged $k_{Q_{i}}$ times,

$$
h_{V}\left(Q_{i}\right)=l_{Q_{i}}-\frac{1}{3} k_{Q_{i}} \quad \text { and } \quad h_{V}\left(Q_{i}\right)+h_{E}\left(Q_{i}\right)=\frac{3}{2} l_{Q_{i}}-\frac{1}{3} k_{Q_{i}}+1 .
$$

Then Equation (5.3) follows and $r\left(Q_{i}\right) \geq \frac{4}{7}$ if $k_{Q_{i}} \leq l_{Q_{i}}-4$.

Note that each $B_{0}$-edge is contained in at most one odd circuit of $F$. Thus, it can be discharged at most once. For each $Q_{i} \in \mathcal{Q}_{I I}$, we have the following result.

\section{Claim 33.}

$$
\sum_{Q_{i} \in \mathcal{Q}_{I I}} h_{V}\left(Q_{i}\right)=\frac{4}{7}\left(\sum_{Q_{i} \in \mathcal{Q}_{I I}} h_{V}\left(Q_{i}\right)+\sum_{Q_{i} \in \mathcal{Q}_{I I}} h_{E}\left(Q_{i}\right)\right)+\frac{2}{7}\left|B_{0}-B_{0}^{\prime}\right|+\frac{1}{7}\left|B_{0}^{\prime}\right|,
$$

where $B_{0}^{\prime}$ is the set of $B_{0}$-edges with one endvertex being discharged once. 
Proof. For each $Q_{i} \in \mathcal{Q}_{I I}$ with $b\left(Q_{i}\right) \in B_{0}^{\prime}$, we have

$$
h_{V}\left(Q_{i}\right)=2-\frac{1}{3}=\frac{5}{3} \quad \text { and } \quad h_{V}\left(Q_{i}\right)+h_{E}\left(Q_{i}\right)=3-\frac{1}{3}=\frac{8}{3} .
$$

Hence,

$$
h_{V}\left(Q_{i}\right)=\frac{4}{7}\left(h_{V}\left(Q_{i}\right)+h_{E}\left(Q_{i}\right)\right)+\frac{1}{7} .
$$

Similarly, for each $Q_{i} \in \mathcal{Q}_{I I}$ with $b\left(Q_{i}\right) \in B_{0}-B_{0}^{\prime}$, we have

$$
h_{V}\left(Q_{i}\right)=\frac{4}{7}\left(h_{V}\left(Q_{i}\right)+h_{E}\left(Q_{i}\right)\right)+\frac{2}{7} .
$$

Then Equation (5.4) follows from above two equations.

\subsection{The Final Step}

Assume the Discharging Rule (R1) and (R2) occur $x_{1}$ and $x_{2}$ times, respectively. Thus, $\left|B_{0}\right| \geq x_{2}$. By Claim 30, both Equations (5.1) and (5.2) remain the same. Combing with Claims 31, 32, and 33, we have

$$
\begin{aligned}
n & =\sum_{Q \in \mathcal{Q}_{I}} h_{V}(Q)+\sum_{Q \in \mathcal{Q}_{I I}} h_{V}(Q)+\sum_{Q \in \mathcal{Q}_{I I I}} h_{V}(Q) \\
& =\frac{4}{7}\left(\sum_{Q \in \mathcal{Q}} h_{V}(Q)+\sum_{Q \in \mathcal{Q}} h_{E}(Q)\right)+\frac{1}{7} \sum_{Q \in \mathcal{Q}_{I}}\left(l_{Q}-k_{Q}-4\right)+\frac{2}{7}\left(\left|B_{0}\right|-x_{2}\right)+\frac{1}{7} x_{2} \\
& =\frac{4}{7}\left(n+|R|+\left|R^{*}\right|\right)+\frac{1}{7}\left(\sum_{Q \in \mathcal{Q}_{I}} l_{Q}-\sum_{Q \in \mathcal{Q}_{I}} k_{Q}-\sum_{Q \in \mathcal{Q}_{I}} 4\right)+\frac{1}{7}\left(2\left|B_{0}\right|-x_{2}\right)
\end{aligned}
$$

Note that

$$
\sum_{Q \in \mathcal{Q}_{I}} l_{Q}=\sum_{\mu \geq 2} 2 \mu t_{2 \mu}, \quad \sum_{Q \in \mathcal{Q}_{I}} k_{Q}=x_{1}, \quad \text { and } \quad \sum_{Q \in \mathcal{Q}_{I}} 1=\sum_{\mu \geq 2} t_{2 \mu} .
$$

Since $n=|E(F)|$ and $|E(G)|=|E(F)|+|R|$, it follows from Equations (5.5) and (5.6) that

$$
|E(F)|=\frac{4}{7}|E(G)|+\frac{4}{7}\left|R^{*}\right|+\frac{1}{7}\left(\left(\sum_{\mu \geq 2}(2 \mu-4) t_{2 \mu}\right)-x_{1}\right)+\frac{1}{7}\left(2\left|B_{0}\right|-x_{2}\right) .
$$


By Claim 27 and the Discharging Rule, for each $Q \in \mathcal{Q}_{I}$, we have $k_{Q} \leq \frac{l_{Q}}{2}-1$ if $l_{Q} \geq 6$ and $k_{Q}=0$ otherwise. It follows from Equation (5.6) that

$$
x_{1}=\sum_{Q \in \mathcal{Q}_{I}} k_{Q} \leq \sum_{Q \in \mathcal{Q}_{I}: l_{Q} \geq 6}\left(\frac{l_{Q}}{2}-1\right)=\sum_{\mu \geq 3}(\mu-1) t_{2 \mu} \leq \sum_{\mu \geq 3}(2 \mu-4) t_{2 \mu}
$$

That is,

$$
\left(\sum_{\mu \geq 2}(2 \mu-4) t_{2 \mu}\right)-x_{1}=\left(\sum_{\mu \geq 3}(2 \mu-4) t_{2 \mu}\right)-x_{1} \geq 0 .
$$

It follows from Equation (5.7) that

$$
|E(F)| \geq \frac{4}{7}|E(G)|+\frac{4}{7}\left|R^{*}\right|+\frac{1}{7}\left(2\left|B_{0}\right|-x_{2}\right) .
$$

Since $\left|B_{0}\right| \geq\left|V_{2}(G)\right|$ and $\left|B_{0}\right| \geq x_{2}$, we have

$$
\left(2\left|B_{0}\right|-x_{2}\right) \geq\left|B_{0}\right| \geq\left|V_{2}(G)\right| .
$$

Note that $\left|R^{*}\right| \geq 1$. Thus,

$$
|E(F)| \geq \frac{4}{7}(|E(G)|+1)+\frac{1}{7}\left|V_{2}(G)\right| .
$$

This last inequality contradicts the choice of $(G, F)$. 


\section{Bibliography}

[1] N. Alon, M. Tarsi, Covering multigraphs by simple circuits, SIAM J. Algebraic Discrete Methods 6 (1985) 345-350.

[2] B. Alspach, L.A. Goddyn, C.-Q. Zhang, Graphs with the circuit cover property, Trans. Amer. Math. Soc. 344 (1994) 131-154.

[3] J.C. Bermond, B. Jackson, F. Jaeger, Shortest covering of graphs with cycles, J. Combin. Theory Ser. B 35 (1983) 297-308.

[4] J.A. Bondy, U.S.R. Murty, Graph Theory, Springer, 2008.

[5] A. Bouchet, Nowhere-zero Integral Flows on a Bidirected Graph, J. Combin. Theory Ser. B 34 (1983) 279-292.

[6] F. Chen, G. Fan, Maximum even factors of graphs, J. Combin. Theory Ser. B 119 (2016) 237-244.

[7] M. DeVos, Flows on Bidirected Graphs, arXiv:1310.8406 (October 2013).

[8] R. Diestel, Graph Theory, Fourth edn. Springer-Verlag, 2010.

[9] J. Edmonds, Maximum Matching and a Polyhedron with 0,1-Vertices, J. Res. Nat. Bur. Standards B 69 (1965) 125-130.

[10] G. Fan, Short cycle covers of cubic graphs, J. Graph Theory 18 (1994) 131-141.

[11] G. Fan, A. Raspaud, Fulkerson's conjecture and circuits covers, J. Combin. Theory Ser. B 61 (1994) 133-138. 
[12] G. Fan, Proofs of two minimum circuit cover conjectures, J. Combin. Theory Ser. B 74 (1998) 353-367.

[13] O. Favaron, M. Kouider, Even Factors of Large Size, J. Graph Theory 77 (2014) 58-67.

[14] H. Fleischner, Eine gemeinsame Basis für die Theorie der eulerschen Graphen und den Satz von Petersen. Monatsh. Math. 81 (1976) 267-278.

[15] H. Fleischner, Eulerian Graphs and Related Topics, Part 1, Vol. 1, Ann. Discrete Math. 45 (1990) North-Holland.

[16] H. Fleischner, Spanning eulerian subgraph, the splitting lemma, and Petersen's theorem, Discrete Math. 101 (1992) 33-37.

[17] A. Itai, M. Rodeh, Covering a graph by circuits, Automata, Languages and Programming, Lecture Notes in Computer Science, vol. 62, Springer, Berlin (1978) 289-299.

[18] B. Jackson, Shortest circuit covers and postman tours of graphs with a nowhere-zero 4-flow, SIAM J. Comput. 19 (1990) 659-665.

[19] B. Jackson, Shortest circuit covers of cubic graphs, J. Combin. Theory Ser. B 60 (1994) 299-307.

[20] F. Jaeger, Flows and generalized coloring theorems in graphs, J. Combin. Theory Ser. B 26 (1979) 205-216.

[21] F. Jaeger, On circular flows in graphs, in Finite and Infinite Sets (Eger, 1981), Colloquia Mathematica Societatis Janós Bolyai, vol.37, North-Holland, AmsterdamNew-York, (1984) 391-402.

[22] K. Jain, Open Problems Garden, http://www.openproblemgarden.org/op/unit_ vector_flows, Reference accessed on July 9, 2017.

[23] U. Jamshy, A. Raspaud, M. Tarsi, Short circuit covers for regular matroids with nowhere-zero 5-flow, J. Combin. Theory Ser. B 43 (1987) 354-357. 
[24] U. Jamshy, M. Tarsi, Shortest cycle covers and the cycle double cover conjecture, J. Combin. Theory Ser. B 56 (1992) 197-204.

[25] T. Kaiser, E. Rollová, Nowhere-zero flows in signed series-parallel graphs, SIAM J. Discrete Math. 30(2) (2016) 1248-1258.

[26] T. Kaiser, R. Lutot'ka, E. Rollová, Nowhere-zero flows in signed graphs: A survey, arXiv:1608.06944 (August 2016).

[27] A. Khelladi, Nowhere-zero integral chains and flows in bidirected graphs, J. Combin. Theory Ser. B 43 (1987) 95-115.

[28] A.V. Kostochka, The $\frac{7}{5}$-conjecture strengthens itself, J. Graph Theory 19 (1995) 65-67.

[29] H.-J. Lai, Z.-H. Chen, Even subgraphs of a graph, Combinatorics, Graph Theory and Algorithms New Issues Press, Kalamazoo (1999) 221-226.

[30] L. Lovász, Subgraphs with Prescribed Valencies, J. Combin. Theory Ser. B 8 (1970) 391-416.

[31] L. Lovász, Three Short Proofs in Graph Theory, J. Combin. Theory Ser. B 19 (1975) 269-271.

[32] L.M. Lovász, C. Thomassen, Y.-Z. Wu, C.-Q. Zhang, Nowhere-zero 3-flow and modulo k-orientation, J. Combin. Theory Ser. B 103 (2013) 587-598.

[33] Y. Lu, R. Luo, C.-Q. Zhang, Flows of signed graphs with low edge connectivity, submitted.

[34] E. Máčajová, M. Škoviera, Characteristic flows on signed graphs and short circuit covers, Electronic Journal of Combinatorics 23(3) (2016) P3.30.

[35] W. Mader, A reduction method for edge-connectivity in graphs, Ann. Discrete Math. 3 (1978) 145-164.

[36] E. Máčajová, A. Raspaud, E. Rollová, M. Škoviera, Circuit covers of signed graphs, J. Graph Theory 81 (2016) 120-133. 
[37] E. Máčajová, A. Raspaud, M. Tarsi, X.-D. Zhu, Short cycle covers of graphs and nowhere-zero flows, J. Graph Theory 68 (2011) 340-348.

[38] C. St. J. A. Nash-Williams, Connected detachments of graphs and generalized Euler trials, J. London Math. Soc. (2) 31 (1985) 17-29.

[39] J. Petersen, Die Theorie der regul'aren graphs, Acta Mathematica 15 (1991) 193-220.

[40] A. Raspaud, X.-D. Zhu, Circular flow on signed graphs, J. Combin. Theory Ser. B 101 (2011) 464-479.

[41] M. Schubert, E. Steffen, Nowhere-zero flows on signed regular graphs, European J. Combin. 48 (2015) 34-47.

[42] P.D. Seymour, Nowhere-Zero 6-Flows, J. Combin. Theory Ser. B 30 (1981) 130-135.

[43] Z. Szigeti, Edge-connectivity augmentation of graphs over symmetric parity families, Discrete Math. 308 (2008) 6527-6532.

[44] C. Thomassen, The weak 3 -flow conjecture and the weak circular flow conjecture, J. Combin. Theory Ser. B 102 (2012) 521-529.

[45] C. Thomassen, Group flow, complex flow, unit vector flow and the $(2+\epsilon)$-flow conjecture, J. Combin. Theory Ser. B 108 (2014) 81-91.

[46] W.T. Tutte, The Factorization of Linear Graphs, J. London Math. Soc. 22 (1947) 107-111.

[47] W.T. Tutte, On the imbedding of linear graphs in surfaces, Proc. London Math. Soc. (2) $\mathbf{5 1}$ (1949) 474-483.

[48] W.T. Tutte, The factors of graphs, Canad. J. Math. 4 (1952) 314-328.

[49] W.T. Tutte, A contribution to the theory of chromatic polynomials, Canad. J. Math. 6 (1954) 80-91.

[50] D.B. West, Introduction to Graph Theory, Upper Saddle River, NJ: Prentice Hall, 1996. 
[51] R. Xu, C.-Q. Zhang, On flows in bidirected graphs, Discrete Math. 299 (2005) 335343.

[52] C.-Q. Zhang, Minimum cycle coverings and integer flows, J. Graph Theory 14 (1990) 537-546.

[53] C.-Q. Zhang, Integer Flows and Cycle Covers of Graphs, Marcel Dekker Inc., New York, 1997.

[54] C.-Q. Zhang, Circular flows of nearly eulerian graphs and vertex-splitting, J. Graph Theory 40 (2002) 147-161.

[55] C.-Q. Zhang, Circuit Double Covers of Graphs, Cambridge University Press, 2012.

[56] X. Zhu, Circular flow number of highly edge connected signed graphs J. Combin. Theory Ser. B 112 (2015) 93-103.

[57] O. Zýka, Nowhere-zero 30-flow on bidirected graphs, Thesis, Charles University, Praha, 1987. 